\title{
Gravitational Waves from Gravitational Collapse
}

\author{
Chris L. Fryer \\ Los Alamos National Laboratory \\ MS D409, CCS-2 \\ Los Alamos, New Mexico 87545, U.S.A. \\ email: fryer@lanl.gov \\ Kimberly C. B. New \\ Los Alamos National Laboratory \\ MS T087, X-4 \\ Los Alamos, New Mexico 87545, U.S.A. \\ email: knew@lanl.gov
}

Accepted on 14 January 2011

Published on 20 January 2011

\begin{abstract}
Gravitational-wave emission from stellar collapse has been studied for nearly four decades. Current state-of-the-art numerical investigations of collapse include those that use progenitors with more realistic angular momentum profiles, properly treat microphysics issues, account for general relativity, and examine non-axisymmetric effects in three dimensions. Such simulations predict that gravitational waves from various phenomena associated with gravitational collapse could be detectable with ground-based and space-based interferometric observatories. This review covers the entire range of stellar collapse sources of gravitational waves: from the accretion-induced collapse of a white dwarf through the collapse down to neutron stars or black holes of massive stars to the collapse of supermassive stars.
\end{abstract}

This review is licensed under a Creative Commons Attribution-Non-Commercial-NoDerivs 3.0 Germany License. http://creativecommons.org/licenses/by-nc-nd/3.0/de/ 


\section{Imprint / Terms of Use}

Living Reviews in Relativity is a peer reviewed open access journal published by the Max Planck Institute for Gravitational Physics, Am Mühlenberg 1, 14476 Potsdam, Germany. ISSN 1433-8351.

This review is licensed under a Creative Commons Attribution-Non-Commercial-NoDerivs 3.0 Germany License: http://creativecommons.org/licenses/by-nc-nd/3.0/de/

Because a Living Reviews article can evolve over time, we recommend to cite the article as follows:

Chris L. Fryer and Kimberly C. B. New,

"Gravitational Waves from Gravitational Collapse", Living Rev. Relativity, 14, (2011), 1. [Online Article]: cited [<date $>$, http://www.livingreviews.org/lrr-2011-1

The date given as $<$ date $>$ then uniquely identifies the version of the article you are referring to. 


\section{Article Revisions}

Living Reviews supports two ways of keeping its articles up-to-date:

Fast-track revision A fast-track revision provides the author with the opportunity to add short notices of current research results, trends and developments, or important publications to the article. A fast-track revision is refereed by the responsible subject editor. If an article has undergone a fast-track revision, a summary of changes will be listed here.

Major update A major update will include substantial changes and additions and is subject to full external refereeing. It is published with a new publication number.

For detailed documentation of an article's evolution, please refer to the history document of the article's online version at http://www. livingreviews.org/lrr-2011-1.

20 January 2011: We have significantly altered and restructured the text of the previous version, describing the latest results from a new point of view. New figures have been added. The number of references has increased from 271 to 350 . 


\section{Contents}

1 Introduction $\quad 5$

2 Gravitational Wave Emission $\quad 7$

2.1 Making numerical estimates . . . . . . . . . . . . . . . . . . . . . . 10

3 Astrophysical Sources of Gravitational Wave Emission 13

3.1 Core-collapse supernovae . . . . . . . . . . . . . . . . . . . . . . . . . . . . . . . . 19

3.1 Core-collapse supernovae rate . . . . . . . . . . . . . . . . . . 19

3.1.2 Core-collapse evolution . . . . . . . . . . . . . . . . . . 19

3.1.3 Core collapse: Sources of gravitational wave emission . . . . . . . . . . . 23

3.2 Accretion-induced collapse . . . . . . . . . . . . . . . . . . . . 24

3.2 .1 AIC rates . . . . . . . . . . . . . . . . 24

3.2.2 Evolution of AIC . . . . . . . . . . . . . . . . . . . . . . . . . . . . . . .

3.2 .3 AIC: Sources of gravitational wave emission . . . . . . . . . . . . 25

3.3 Electron-capture supernovae and core-collapse supernovae below 10-12 solar masses:

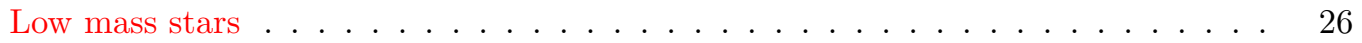

3.3.1 Rate of supernovae from low-mass stars . . . . . . . . . . . . . . . . 26

3.3.2 Low-mass star collapse: Evolution . . . . . . . . . . . . . . . . . . 26

3.3.3 Low-mass star collapse: Gravitational wave emission mechanisms . . . . . . 26

3.4 Core-collapse supernovae from stars above 20 solar masses: Massive star collapse . 27

3.4.1 Massive star collapse: Evolution . . . . . . . . . . . . . . . . . 27

3.4.2 Rates of massive star collapse . . . . . . . . . . . . . . . . . . . . . . 27

3.4.3 Gravitational waves from massive stars . . . . . . . . . . . . . . . . . 29

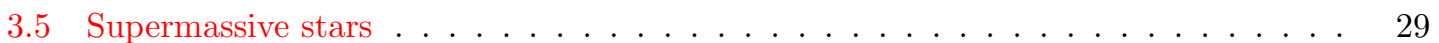

3.5.1 Supermassive stars: Evolution _. . . . . . . . . . . . . . . 29

3.5.2 Rates of supermassive stars . . . . . . . . . . . . . . . . . . 30

3.5.3 Gravitational-wave emission mechanisms of supermassive stars . . . . . . 31

4 Gravitational Wave Emission Mechanisms $\quad 32$

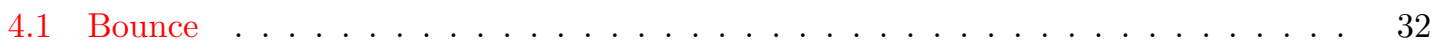

4.1 General relativistic calculations . . . . . . . . . . . . . . 36

4.2 Convection . . . . . . . . . . . . . . . . . . . . . 41

4.2 .1 Asymmetric collapse . . . . . . . . . . . . . . . . . . . . . . . . . . . . .

4.2 .2 Proto neutron star convection . . . . . . . . . . . . . . . . . 41

4.2.3 Convection above the proto neutron star . . . . . . . . . . . . . . . 45

4.2.4 Low-mode convection and the standing accretion-shock instability . . . . . 45

4.3 Bar modes . . . . . . . . . . . . . . . . . . . . . . . . 48

4.3.1 Equilibrium models to study instabilities . . . . . . . . . . . . . 50

4.3.2 Hydrodynamic models . . . . . . . . . . . . . . . . . . . . . . . 51

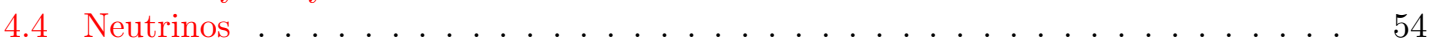

4.5 r-Modes . . . . . . . . . . . . . . . . . . . . 57

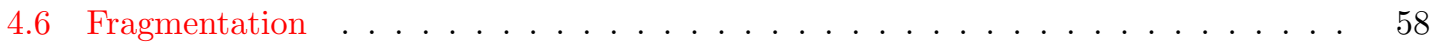

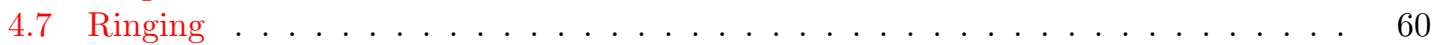

5 Summary $\quad 64$

5.1 Detection of collapse GW signals . . . . . . . . . . . . . . . . . . . . . 64

5.2 Using GWs to study core collapse . . . . . . . . . . . . . . . . . . 67

$\begin{array}{llr}6 & \text { Acknowledgements } & 68\end{array}$

$\begin{array}{lr}\text { References } & 69\end{array}$ 


\section{Introduction}

The field of gravitational-wave (GW) astronomy will soon become a reality. The first generation of ground-based interferometric detectors (LIGO [177], VIRGO [324], GEO600 [122], TAMA300 [301]) are beginning their search for GWs. Upgrades for two of these detectors (LIGO, VIRGO) have already begun and in the next few years, the sensitivity of both these detectors will be increased. In addition, these two detectors have begun working more closely together, improving their net sensitivity. After these detectors are complete, they will have sensitivities necessary to regularly detect emissions from astrophysical sources. New detectors, such as the Large-Scale Cryogenic Gravitational-wave Telescope (LCGT) as well as technology developing sites such as the Australian Interferometric Gravitational Observatory (AIGO) are also contributing to the progress in GW astronomy (see [145] for a review). A space-based interferometric detector, LISA [182], could be launched in the next decade. One important class of sources for these observatories is stellar gravitational collapse. This class covers an entire spectrum of stellar masses, from the accretioninduced collapse (AIC) of a white dwarf and the collapse of low-mass stars, including electroncapture supernovae $\left(M_{\mathrm{star}}<10 M_{\odot}\right)$, through the collapse of massive stars $\left(M>10 M_{\odot}\right)$ that produce and the even more massive stars $\left(M>20 M_{\odot}\right)$ that produce the "collapsar" engine believed to power long-duration gamma-ray bursts [333], massive and very massive Population III stars $\left(M=20-500 M_{\odot}\right)$, and supermassive stars (SMSs, $M>10^{6} M_{\odot}$ ). Some of these collapses result in explosions (Type II, Ib/c supernovae and hypernovae) and all leave behind neutron-star or black-hole remnants ${ }^{1}$.

Strong GWs can be emitted during a gravitational collapse/explosion and, following the collapse, by the resulting compact remnant [309, 205, 206, 88, 269, 108, 150]. GW emission during the collapse itself may result if the collapse or explosion involves aspherical bulk mass motion or convection. Rotational or fragmentation instabilities encountered by the collapsing star will also produce GWs. Pulsations and instabilities in the newly formed neutron star (a.k.a. proto neutron star) may also produce observable GWs. Asymmetric neutrino emission can also produce a strong GW signature. Neutron-star remnants of collapse may emit GWs due to the growth of rotational or $r$-mode instabilities. Black-hole remnants will also be sources of GWs if they experience accretioninduced ringing or if the disks around the black hole develop instabilities. All of these phenomena have the potential of being detected by GW observatories because they involve the rapid change of dense matter distributions.

Observation of gravitational collapse by GW detectors will provide unique information, complementary to that derived from electromagnetic and neutrino detectors. Gravitational radiation arises from the coherent superposition of mass motion, whereas electromagnetic emission is produced by the incoherent superposition of radiation from electrons, atoms, and molecules. Thus, GWs carry different kinds of information than other types of radiation. Furthermore, electromagnetic radiation interacts strongly with matter and thus gives a view of the collapse only from lower density regions near the surface of the star, and it is weakened by absorption as it travels to the detector. In contrast, gravitational waves can propagate from the innermost parts of the stellar core to detectors without attenuation by intervening matter. With their weak interaction cross-sections, neutrinos can probe the same region probed by GWs. But whereas neutrinos are extremely sensitive to details in the microphysics (equation of state and cross-sections), GWs are most sensitive to physics driving the mass motions (e.g., rotation). Combined, the neutrino and the GW signals can teach us much about the conditions in the collapsing core and ultimately the physics that governs stellar collapse (e.g., [7, 107, 167, 228]).

The characteristics of the GW emission from gravitational collapse have been the subject of much study. Core-collapse supernovae, in particular, have been investigated as sources of gravita-

\footnotetext{
1 Note that some stars in $M=100-500 M_{\odot}$ mass range may not collapse at all, but rather explode in a pair-instability supernovae [136].
} 
tional radiation for nearly four decades (see, e.g., [251, 311, 253, 64, 204, 89, 201, 350, 245, 106, $108,67,68,165,232])$. However, during this time research has produced estimates of GW strength that vary over orders of magnitude. This is due to the complex nature of core collapse. Important theoretical and numerical issues include

- construction of accurate progenitor models, including realistic angular momentum distributions,

- proper treatment of microphysics, including the use of realistic equations of state and neutrino transport,

- simulation in three-dimensions to study non-axisymmetric effects,

- inclusion of general-relativistic effects,

- inclusion of magnetic-field effects, and

- study of the effect of an envelope on core behavior.

To date, collapse simulations generally include state-of-the-art treatments of only one or two of the above physics issues (often because of numerical constraints). For example, those studies that include advanced microphysics have often been run with Newtonian gravity (and approximate evaluation of the GW emission; see, e.g., Section 4.1). Very few, if any simulations, have reached any convergence in spatial resolution. Many of the codes have not been tested to see if their algorithm implementations guard against standard numerical artifacts. For example, very few codes used have tested the effects of the non-conservation of angular momentum and the numerical transport of this angular momentum. A 3D, general relativistic collapse simulation that includes all significant physics effects is not feasible at present. However, good progress has been made on the majority of the issues listed above; the more recent work will be reviewed in some detail here.

The remainder of this article is structured as follows. We first review the basic modes of GW emission in stellar collapse presenting, where they exist, analytic formulae that have been used to estimate these GWs (Section 2). The latter half of Section 2 presents many of the numerical approximations used to calculate GW emission. Section 3 covers the various collapse scenarios and their GW sources: normal core-collapse supernovae (Section 3.1), the accretion induced collapse (AIC) of a white dwarf (Section 3.2), the collapse of low mass stars and electron capture supernovae (Section 3.3), and the collapse of massive (Section 3.4) and supermassive (Section 3.5) stars. For each, we review the current understanding of the occurrence rate, collapse evolution, and the specific causes behind GW emission. Section 4 then discusses the current state of the calculations for sources arising from: bounce (Section 4.1), convection (Section 4.2), bar modes (Section 4.3), neutrinos (Section 4.4), $r$-modes (Section 4.5), fragmentation (Section 4.6), and ringing (Section 4.7). We conclude by tying together all of these sources with their emission mechanisms to predict a complete GW sky.

One final word of warning. In many cases, the total GW signal from stellar collapse can be tuned by altering key initial conditions (such as the rotation rate of the collapsing star). Many of the strongest GW estimates in the literature tend to use rotation rates that are orders of magnitude higher than that predicted for most stars. These more optimistic results often predict that the current set of detectors should observe GWs from astrophysical sources. In many cases, studies of these extreme conditions provide insight into possible mechanisms for GW emission. To include these new insights, we will discuss these results in this review. The exact nature of the initial conditions may make certain GW signals undetectable. For each of the these scenarios, we strive to distinguish academic studies with more realistic estimates of the signal. Our summary is based on what we judge to be the more realistic signal predictions. 


\section{Gravitational Wave Emission}

Most studies of GW emission use a multipole expansion of the perturbation $h_{\mu \nu}$ to a background spacetime $g_{\mu \nu}^{b}$. The lowest (quadrupole) order piece of this field is [306]:

$$
h_{j k}^{\mathrm{TT}}=\left[\frac{2}{d} \frac{G}{c^{4}} \frac{d^{2}}{d t^{2}} \mathcal{I}_{j k}(t-r)+\frac{8}{3 d} \frac{G}{c^{5}} \epsilon_{p q(j} \frac{d^{2}}{d t^{2}} \mathcal{S}_{k) p}(t-r) n_{q}\right]^{\mathrm{TT}} .
$$

Here, $\mathcal{I}_{j k}$ and $\mathcal{S}_{j k}$ are the mass and current quadrupole moments of the source, $d$ is the distance from the source to the point of measurement, $\epsilon_{i j k}$ is the antisymmetric tensor, and $n_{q}$ is the unit vector pointing in the propagation direction. Parentheses in the subscripts indicate symmetrization over the enclosed indices, and the superscript TT indicates that one is to take the transverse-traceless projection; $G$ is Newton's gravitational constant, and $c$ is the speed of light.

Most GW estimates are based on Equation (1). When bulk mass motions dominate the dynamics, the first term describes the radiation. For example, this term gives the well-known "chirp" associated with binary inspiral. It can be used to model bar-mode and fragmentation instabilities. At least conceptually, this term also applies to black hole ringing, provided one interprets $\mathcal{I}_{j k}$ as a moment of the spacetime rather than as a mass moment $[310,162])$. In practice, ringing waves are computed by finding solutions to the wave equation for gravitational radiation [303] with appropriate boundary conditions (radiation purely ingoing at the hole's event horizon, purely outgoing at infinity). The second term in Equation (1) gives radiation from mass currents, and is used to calculate GW emission due to the $r$-mode instability.

When the background spacetime is flat (or nearly so) the mass and current moments have particularly simple forms. For example, in Cartesian coordinates the mass quadrupole is given by

$$
\mathcal{I}_{j k}=\int d^{3} x \rho\left[x^{j} x^{k}-\frac{1}{3} r^{2} \delta_{j k}\right],
$$

where $\rho$ is the mass density, and $\delta_{j k}=1$ for $j=k$ and 0 otherwise. The second term in the integrand ensures that the resulting tensor is trace free.

There are several phases during the collapse (and resultant explosion) of a star that produce rapidly changing quadrupole moments: both in the baryonic matter and in the neutrino emission. We will break these phases into four separate aspects of the core-collapse explosion: the bounce of the core, the convective phase above the proto neutron star, cooling in the neutron star, and formation phase of a black hole. Scientists have focused on different phases at different times, arguing that different phases dominated the GW emission. These differences are primarily due to different initial conditions. A specific stellar collapse will only pass through a subset of these phases and the magnitude of the GW emission from each phase will also vary with each stellar collapse. Fryer, Holz \& Hughes (hereafter FHH) [106] reviewed some of these phases and calculated upper limits to the GWs produced during each phase [106]. Recent studies have confirmed these upper limits, typically predicting results between $5-50$ times lower than the secure FHH upper limits $[165,228]$. Before we discuss simulations of GWs, we review these FHH estimates and construct a few estimates for phases missed by FHH.

Those collapsing systems that form neutron stars (all but the most massive stellar collapses) will emit a burst of GWs at bounce if there is an asymmetry in the bounce (either caused by rotation or by an asymmetry in the stellar core prior to collapse). If we represent the matter asymmetry in the collapse by a point mass being accelerated as the core bounces, we can estimate the GW signal from the bounce phase (using, for example, Equations (15)-(19)):

$$
<\left(r h_{+}\right)^{2}>^{1 / 2} \approx G / c^{4} \sqrt{8 / 3}\left(a_{p} x_{p}+v_{p}^{2}\right) m_{p},
$$


where $a_{p}$ is the acceleration, $x_{p}$ is the position (providing a lever arm for the GW emission), $v_{p}$ is the velocity, and $m_{p}$ is the mass of the parcel of matter driving the GW emission. If we limit our estimate to the acceleration term, we find

$$
<\left(r h_{+}\right)^{2}>^{1 / 2} \approx 50\left(a_{p} / 6 \times 10^{11} \mathrm{~cm} \mathrm{~s}^{-2}\right)\left(x_{p} / 30 \mathrm{~km}\right)\left(m_{p} / 0.1 M_{\odot}\right) .
$$

For a $0.1 M_{\odot}$ asymmetry, where the matter at $30 \mathrm{~km}$ is accelerated from $-0.1 c$ to $0.1 c$ in $10 \mathrm{~ms}$ (typical for a core-collapse bounce), the signal would be roughly $16 \times 10^{-22}$ at $10 \mathrm{kpc}$. Although one could imagine increasing this signal by including the velocity term, it is unlikely that we will be able to increase this value by more than an order of magnitude.

This formulation can also be used to estimate the GW emission for convection. After bounce, convection begins to grow within and on top of the proto neutron $\operatorname{star}^{2}$. In this extreme case, the GW signal convection can once again be estimated by a point source accelerating across the convective regime. Here the acceleration of the matter occurs over a $100 \mathrm{~ms}$ timescale and the velocities are peaking at roughly $0.01 \mathrm{c}$. As such, we expect convective motion alone to be an order of magnitude lower than bounce:

$$
<\left(r h_{+}\right)^{2}>^{1 / 2} \approx 5\left(a_{p} / 6 \times 10^{9} \mathrm{~cm} \mathrm{~s}^{-2}\right)\left(x_{p} / 100 \mathrm{~km}\right)\left(m_{p} / 0.3 M_{\odot}\right) .
$$

That is, for typical convective regions, we expect a signal of less than $1.6 \times 10^{-22}$ at $10 \mathrm{kpc}$. Any post-bounce signal stronger than this result is likely to arise from additional instabilities such as bar modes.

FHH focused on three emission sources after the bounce. In the convective regime, they studied the development of two different instabilities: fragmentation and bar modes. Bar modes can also develop in the neutron star as it cools. FHH [106] also estimated the $r$-mode strength in neutron stars. Let's review these estimates.

Fragmentation can only occur in very rapidly-rotating models. Currently, the predicted rotation rates of stellar models are simply not fast enough to develop such instabilities [245, 113, 282]. However, the argument that massive accretion disks act as gamma-ray burst (GRB) engines [242] opens up a new scenario for fragmentation instabilities. Van Putten [320] has argued that fragmentationtype instability can occur in black-hole-forming collapses, if it is rotating sufficiently fast to form a massive disk. Thus far, simulations of such disks have developed Rossby-like instabilities, but these instabilities do not lead to true fragmentation. Requiring $\Omega c_{s} / \pi G \Sigma<1[316,123]$ is a necessary, but not sufficient condition [239]; see Zink et al. [348]. Assuming they can occur, GW estimates of fragmentation provide a strong upper limit to the GW signal. In Keplerian orbits, with equal massed fragments, this limit is $(\mathrm{FHH})$ :

$$
\begin{gathered}
P_{\text {fragment }}=2 / 5 G^{4} / c^{5} m^{5} / r^{5}, \\
h_{\text {fragment }}=\sqrt{8 / 5} G^{2} / c^{4} m^{2} /(r d),
\end{gathered}
$$

where $G$ is the gravitational constant, $c$ is the speed of light, $m$ is the mass of the fragments, $r$ is their separation, and $d$ is the distance to the detector. In the black-hole forming case, when the "horizons" of the two fragments touch, the power radiated reaches a maximum: $P=$ $c^{5} /(80 G) \sim 10^{57} \mathrm{ergs} \mathrm{s}^{-1}$. This is a strong source, if simulations confirm that such fragments exist. For black holes, fragmentation will lead to clump accretion and black hole ringing.

Considerably more effort has been focused on bar modes, or more generally, non-axisymmetric instabilities. Classical, high $\beta$ (where $\beta$ is the ratio of the rotational to potential energy $\equiv T /|W|$ ),

\footnotetext{
${ }^{2}$ Currently, there are major discussions on the details of the drive in the convection above the proto neutron star. Instabilities can be driven by the post-bounce-shock entropy profile, neutrino heating, or the standing accretion shock instability (SASI). Similarly, debate surrounds the magnitude of the entropy/lepton driven convection within the proto neutron star.
} 
bar modes can be separated into two classes: dynamical instabilities and secular instabilities. The classical analysis predicts that $\beta \gtrsim 0.27$ is needed for dynamical instabilities and $(\beta \gtrsim 0.14)$ is required for secular instabilities. Collapse calculations of many modern progenitors (e.g., [138]) followed through collapse calculations [245, 113, 69] suggest that such "classical" instabilities do not occur in nature.

However, advances in both progenitors and non-axisymmetric instabilities have revived the notion of bar modes. First, the search for progenitors of GRBs has led stellar and binary modelers to devise a number of collapse scenarios with rapidly-rotating cores: mixing in single stars [338, 340], binary scenarios [114, 240, 104, 319, 42], and the collapse of merging white dwarfs [341, 339, 342, $118,63,61]$. Second, calculations including differentially-rotating cores also discovered that, with differential rotation, instabilities can occur at much lower values for $\beta$, as low as 0.01 [278, 279]. We will discuss these new discoveries in detail in Section 4.3. FHH estimated the upper limits of the GW signals from bar-instabilities assuming a simple single bar of length $2 r$ :

$$
\begin{gathered}
P_{\mathrm{bar}}=\frac{32}{45} \frac{G}{c^{5}} m^{2} r^{4} \omega^{6} \\
h_{\mathrm{bar}}=\sqrt{32 / 45} G / c^{4} m r^{2} \omega^{2} / d .
\end{gathered}
$$

Lastly, there has been considerable discussion about the existence of $r$-modes in the cooling proto neutron star $[4,96]$. FHH estimated an upper limit for the $r$-mode signature by using the method of Ho and Lai [143] (which assumes $\alpha_{\max }=1$ ) and calculated only the emission from the dominant $m=2$ mode. This approach is detailed in [106]. If the neutron star mass and initial radius are taken to be $1.4 M_{\odot}$ and $12.53 \mathrm{~km}$, respectively, the resulting formula for the average GW strain is

$$
h(t)=1.8 \times 10^{-24} \alpha\left(\frac{\nu_{\mathrm{s}}}{1 \mathrm{kHz}}\right)\left(\frac{20 \mathrm{Mpc}}{d}\right),
$$

where $\alpha$ is the mode amplitude and $\nu_{\mathrm{s}}$ is the spin frequency. Recent studies suggest that viscosities may be quite high in a cooling proto neutron star, making this source untenable [159, 158, 248, $249,181,178,132,5]$. We will review this argument, and some of the recent progress in estimating the viscosity in a neutron star in Section 4.5. 


\subsection{Making numerical estimates}

In core collapse, estimates of the GW emission from simulations are usually done outside of the actual hydrodynamics calculation - that is, there is no feedback from the loss of energy from GWs from the system. Of course, where the GWs drive or damp an unstable mode (e.g., bar modes, Section 4.3), this approximation will lead to erroneous results. Such an assumption is almost always justified, as the GW emission is many orders of magnitude less than the total energy in the system. Calculating the GW emission from a hydrodynamic simulation requires the discretization of Equation (1) and the discretization used depends upon the numerical technique and the dimension of the calculation. Here we present some common discretizations.

An example of a discretization in spherical coordinates for a 2-dimensional simulation is that used by Müller \& Janka [208] (see also [306, 201]). Here they define the gravitational quadrupole radiation field $\left(h^{\mathrm{TT}}\right)$ :

$$
h_{\theta \theta}=1 / 8 \sqrt{(15 / \pi) \sin ^{2} \theta A_{20}^{\mathrm{E} 2} / r}
$$

where $r$ is the distance between the observer and the source, $\theta$ is the angular coordinate in our $r-\theta$ coordinate system, and $A_{20}^{\mathrm{E} 2}$ is given by:

$$
\begin{aligned}
A_{20}^{\mathrm{E} 2} & =\left(G / c^{4}\right)\left(16 \pi^{3 / 2} / \sqrt{15}\right) \int_{-1}^{1} \int_{0}^{\infty} \rho(r, \mu, t)\left[v_{r} v_{r}\left(3 \mu^{2}-1\right)+v_{\theta} v_{\theta}\left(2-3 \mu^{2}\right)-v_{\phi} v_{\phi}\right. \\
& \left.-6 v_{r} v_{\theta} \mu \sqrt{1-\mu^{2}}+r \partial \Phi / \partial r\left(3 \mu^{2}-1\right)+3 \partial \Phi / \partial \theta \mu \sqrt{1-\mu^{2}}\right] r^{2} d r d \mu
\end{aligned}
$$

where $\mu=\cos \theta, v_{r}, v_{\theta}, v_{\phi}$ are the velocities along the ordinate axes in spherical coordinates and $\Phi$ is the Newtonian potential. In spherical coordinates, the partial derivatives in $\theta$ and $r$ are easily defined $^{3}$ and the integrals simply become summations over the grid space.

In three dimensions, we can simplify this equation:

$$
h_{i j}^{\mathrm{TT}}(\mathbf{X}, t)=1 / R\left[\left(d^{2} I_{x x} / d t^{2}-d^{2} I_{y y} / d t^{2}\right) \mathbf{e}_{+}+2 d^{2} I_{x y} / d t^{2} \mathbf{e}_{\mathbf{x}}\right]
$$

where $\mathbf{e}_{+}=e_{\theta} \otimes e_{\theta}-e_{\phi} \otimes e_{\phi}$ and $\mathbf{e}_{\mathbf{x}}=e_{\theta} \otimes e_{\theta}+e_{\phi} \otimes e_{\phi}$, where

$$
d^{2} I_{i j} / d t^{2}=G / c^{4} \int d^{3} x \rho\left(2 v_{i} v_{j}-x_{i} \partial_{j} \Phi / \partial j-x_{j} \partial \Phi / \partial i\right)
$$

where $\mathrm{i}$ and $\mathrm{j}$ are the coordinate axes.

For smooth particle hydrodynamics ( $\mathrm{SPH}$ ), this formalism becomes [45]:

$$
\begin{aligned}
c^{8} / G^{2}\left\langle\left(r h_{+}\right)^{2}\right\rangle & =\frac{4}{15}\left(\ddot{I}_{x x}-\ddot{I}_{z z}\right)^{2}+\frac{4}{15}\left(\ddot{I}_{y y}-\ddot{I}_{z z}\right)^{2}+\frac{1}{10}\left(\ddot{I}_{x x}-\ddot{I}_{y y}\right)^{2} \\
& +\frac{14}{15}\left(\ddot{I}_{x y}\right)^{2}+\frac{4}{15}\left(\ddot{I}_{x z}\right)^{2}+\frac{4}{15}\left(\ddot{I}_{y z}\right)^{2}, \\
c^{8} / G^{2}\left\langle\left(r h_{\times}\right)^{2}\right\rangle & =\frac{1}{6}\left(\ddot{I}_{x x}-\ddot{I}_{y y}\right)^{2}+\frac{2}{3}\left(\ddot{I}_{x y}\right)^{2}+\frac{4}{3}\left(\ddot{I}_{x z}\right)^{2}+\frac{4}{3}\left(\ddot{I}_{y z}\right)^{2},
\end{aligned}
$$

where $r$ is the distance to the source. The angle brackets in these equations denote averaging over all source orientations, so that for example

$$
\left\langle\left(r h_{+}\right)^{2}\right\rangle=\frac{1}{4 \pi} \int d \Omega r^{2} h_{+}(\theta, \phi)^{2} .
$$

3 Müller \& Janka [208] did not specifically describe what they used, but any standard derivative discretization would work, e.g., $\partial \Phi / \partial r=\left(\Phi^{k+1}-\Phi^{k-1}\right) /\left(r^{k+1}-r^{k-1}\right)$. 
The quantities $\ddot{I}_{i j}$ are the second time derivatives of the trace-free quadrupole moment of the source. The diagonal elements are given by, for example,

$$
\ddot{I}_{x x}=\frac{2}{3} \sum_{p=1}^{N} m_{p}\left(2 x_{p} \ddot{x}_{p}-y_{p} \ddot{y}_{p}-z_{p} \ddot{z}_{p}+2 \dot{x}_{p}^{2}-\dot{y}_{p}^{2}-\dot{z}_{p}^{2}\right),
$$

where $m_{p}$ is the mass of an SPH particle, and $\left(x_{p}, y_{p}, z_{p}\right)$ is its coordinate location. The other diagonal elements can be obtained using Equation (18) plus cyclic permutation of the coordinate labels: $\ddot{I}_{x x} \rightarrow \ddot{I}_{y y}$ via $x \rightarrow y, y \rightarrow z, z \rightarrow x ; \ddot{I}_{x x} \rightarrow \ddot{I}_{z z}$ via $x \rightarrow z, z \rightarrow y, y \rightarrow x$. The off-diagonal elements are given by, for example,

$$
\ddot{I}_{x y}=\sum_{p=1}^{N} m_{p}\left(x_{p} \ddot{x}_{p}+y_{p} \ddot{y}_{p}+2 \dot{x}_{p} \dot{y}_{p}\right) ;
$$

the remaining off-diagonal elements can be found via symmetry $\left(\ddot{I}_{j i}=\ddot{I}_{i j}\right)$ plus cyclic permutation. This approach provides only averaged square amplitudes, instead of the specific amplitude of the GW signal.

For GWs from neutrinos, many groups use [208] (based on the original analysis of Epstein [76] and Turner [317]):

$$
\begin{aligned}
\left(h_{x x}^{\mathrm{TT}}\right)_{\text {pole }}= & \frac{2 G}{c^{4} r} \int_{-\infty}^{t-R / c} d t^{\prime} \\
& \int_{4 \pi} d \Omega^{\prime}\left(1+\cos \theta^{\prime}\right) \cos \left(2 \phi^{\prime}\right) \frac{d L_{\nu}\left(\Omega^{\prime}, t^{\prime}\right)}{d \Omega^{\prime}}, \\
\left(h_{x x}^{\mathrm{TT}}\right)_{\text {equator }}= & \frac{2 G}{c^{4} r} \int_{-\infty}^{t-R / c} d t^{\prime} \int_{4 \pi} d \Omega^{\prime}\left(1+\sin \theta^{\prime} \cos \phi^{\prime}\right) \\
& \frac{\cos ^{2} \theta^{\prime}-\sin ^{2} \theta^{\prime} \sin ^{2} \phi^{\prime}}{\cos ^{2} \theta^{\prime}+\sin ^{2} \theta^{\prime} \sin ^{2} \phi^{\prime}} \frac{d L_{\nu}\left(\Omega^{\prime}, t^{\prime}\right)}{d \Omega^{\prime}},
\end{aligned}
$$

where $G$ is the gravitational constant, $c$ is the speed of light and $r$ is the distance of the object. $\left(h_{x x}^{\mathrm{TT}}\right)_{\text {pole }}$ denotes the emergent strain for an observer situated along the source coordinate frame's $\mathrm{z}$-axis (or pole) and $\left(h_{x x}^{\mathrm{TT}}\right)_{\text {equator }}$ is the comparable strain for an observer situated perpendicular to this z-axis (or equator).

In $\mathrm{SPH}$ calculations, these expressions reduce to:

$$
\begin{gathered}
\left(h_{x x}^{\mathrm{TT}}\right)_{\text {pole }}=\frac{2 G}{c^{4} R} \sum \Delta t \sum_{p=1}^{N}(1 \pm z / r)\left(2 x^{2} / r^{2}-1\right) \Delta L_{\nu}, \\
\left(h_{x x}^{\mathrm{TT}}\right)_{\text {equator }}=\frac{2 G}{c^{4} R} \sum \Delta t \sum_{p=1}^{N}\left(1 \pm \sqrt{1-z^{2} / r^{2}}(x, y) / r\right) \frac{z^{2} / r^{2}-\sqrt{1-z^{2} / r^{2}}(x, y)^{2} / r^{2}}{z^{2} / r^{2}+\sqrt{1-z^{2} / r^{2}}(x, y)^{2} / r^{2}} \Delta L_{\nu},
\end{gathered}
$$

where $\Delta t$ is the timestep, the summation is over all particles emitting neutrinos that escape this star (primarily, the boundary particles). There are some assumptions about the emission of the neutrinos in this model, but Fryer et al. [107] argued that these assumptions lead to errors smaller than a factor of 2 . The \pm correspond to observers along the positive/negative directions of each axis: for instance, in the polar equation, this corresponds to the positive/negative z-axis. In the 
equatorial region, the $\mathrm{x}, \mathrm{y}$-axis is determined by the choice of $\mathrm{x}$ or $\mathrm{y}$ position for the (x,y) coordinate in the equation.

The signal arising from neutrino emission is an example of a GW burst "with memory", where the amplitude rises from a zero point and ultimately settles down to a value offset from this initial value. The noise sources and optimal data analysis techniques for these signals will differ from, for example, signals from bounce that do not have memory [25, 80].

Other methods have been developed to calculate the GW emission (e.g., Regge variables [328, 121], Zerilli equations [346], Moncrief metric [202], see [214] for a review). Shibata and collaborators (e.g., [277]) have used the gauge-invariant Moncrief variables in flat spacetime:

$$
\begin{aligned}
& R_{l m}^{E}(t, r)=\sqrt{2(l-2) ! /(l+2) !}\left(4 k_{2 l m}+l(l+1) k_{1 l m}\right) \\
& R_{l m}^{O}(t, r)=\sqrt{2(l+2) ! /(l-2) !}\left(2 C_{l m} / r+r \partial / \partial r D_{l m}\right)
\end{aligned}
$$

where

$$
\begin{gathered}
k_{1 l m} \equiv K_{l m}+l(l+1) G_{l m}+2 r \partial / \partial r G_{l m}-2 h_{1 l m} / r \\
k_{2 l m} \equiv H_{l m} / 2+l(l+1) G_{l m}+2 r \partial / \partial r G_{l m}-2 h_{1 l m} / r
\end{gathered}
$$

where $C_{l m}, D_{l m}, K_{l m}, G_{l m}, H_{l m}$ are functions of $r$ and $t$ and are two-sphere integrations with dependencies on the coordinates $r, \phi, \theta$ and the Lorentz factor $\gamma$. Most of these are defined in Shibata \& Nakamura [280]. With these gauge-invariant variables, we can derive the energy emitted in GWs:

$$
d E / d t=r^{2} / 32 \pi \sum_{l, m}\left[\left|\partial R_{l m}^{E} / \partial t\right|^{2}+\left|\partial R_{l m}^{O} / \partial t\right|^{2}\right]
$$

Shibata \& Sekiguchi [282] define a number of other useful quantities for determining GW emission using this formalism. 


\section{Astrophysical Sources of Gravitational Wave Emission}

The type of collapsing star and the explosion mechanism both determine what sources of GWs can occur in a stellar collapse. Before we discuss the different collapses individually and the detailed simulations, let's discuss some of the basics behind stellar collapse and core-collapse supernovae. In this review, the term "stellar collapse" refers to any stellar system that collapses down to a neutron star or black hole. These stellar systems are produced by many different scenarios, but they can be loosely grouped into two categories:

- The collapse of Oxygen-Magnesium-Neon and Carbon Oxygen White Dwarfs pushed beyond the Chandrasekhar limit

- The collapse of Iron or Oxygen-Magnesium-Neon Stellar Cores of massive stars that become too large to support themselves (also roughly at the Chandrasekhar limit).

For both classes of stellar collapse, the core is supported by a combination of thermal and degeneracy pressures. When the mass is too great for these pressures to support the star/core, it begins to compress and heat up. The compression leads to electron capture, neutrino emission, and ultimately, dissociation of the elements. Electron capture reduces the support from degeneracy pressure while Urca processes and dissociation of elements remove thermal support. With less support, the core compresses further, accelerating the rate of electron capture and dissociation, ultimately leading to a runaway implosion. This collapse continues until the matter reaches nuclear densities and the formation of a proto neutron star, or, in the case of massive stars above $100 M_{\odot}$, a proto black hole $[115]^{4}$.

In stellar collapse, it is the structure (density, entropy, rotation) of the core that determines whether a black hole or neutron star is formed ${ }^{5}$ For those stellar collapses that form neutron stars, the structure of the star just beyond the collapsed core is critical in defining the fate of the system. As the collapsed material reaches nuclear densities, nuclear forces and neutron degeneracy pressure halt the collapse and send a bounce shock out through the star. For nearly all systems, this bounce shock stalls. The shock can be revived by neutrinos leaked from the core depositing their energy above the proto neutron star. This is the basis of the neutrino-driven supernova mechanism [56, 19].

Over the past 15 years, scientists studying the supernova engine have focused on convection above the proto neutron star as a crucial piece of physics needed to unlock powerful supernova explosions (Figure 1). To drive an explosion, the convective engine must overcome the ram pressure at the top of the convective region caused by the infalling star [36, 140, 97, 156, 211]. The infalling material creates a cap or lid on top of the convective engine that must be blown off to produce an explosion. The strength of this "lid" is a function of the infalling accretion rate. Fryer [97] used this simple picture and the varying accretion rates of different stars (Figure 2) to determine the fate of massive stars. A supernova occurs if the engine can blow off the lid.

Using this simplified picture, Fryer and collaborators [97, 109, 136] were able to estimate the fate of massive stars. Fryer and Kalogera [109] outlined three fates for the star: neutron-star formation, black-hole formation through fallback after a weak supernova explosion, and prompt (or direct) black-hole formation where no supernova explosion is launched. The "prompt" or "direct" name has led to some confusion. These systems still initially form a proto neutron star. However, they are unable to throw off the infalling lid and thus they collapse down to a black hole without launching a supernova shock. Very massive (above $\sim 300 M_{\odot}$ ) stars might collapse

\footnotetext{
4 The term "proto black hole" is used to describe the large (above $50 M_{\odot}$ ) core produced in the collapse of a massive star. Because of high entropies, the core does not collapse down to nuclear densities but collapses straight to a black hole, never producing a compact proto neutron star at nuclear densities.

5 Our models of these collapsing cores are also sensitive to the numerical modeling of the physics as well as uncertainties in the physics, both leading to the current variety in results between different simulations.
} 


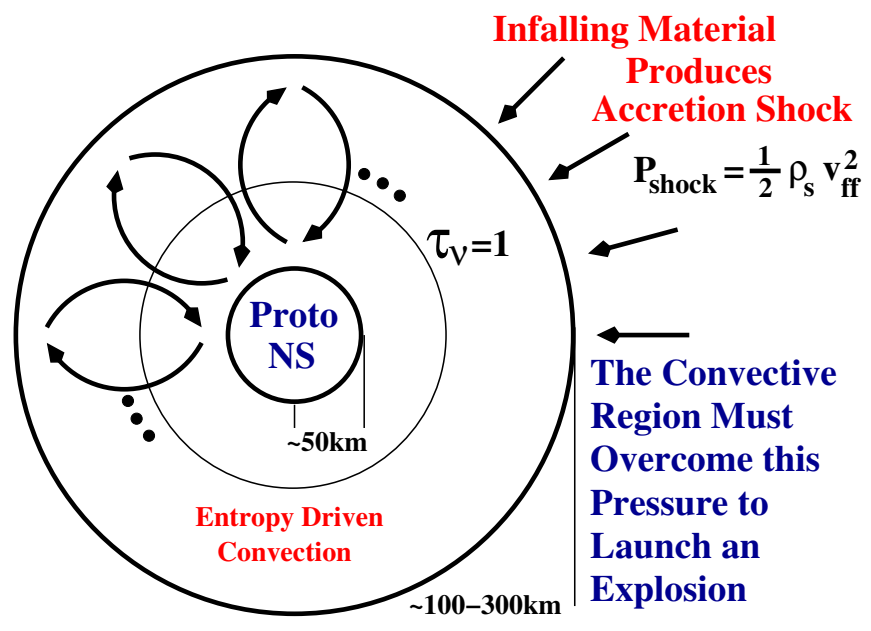

Figure 1: Diagram of the convective engine for supernova explosions.

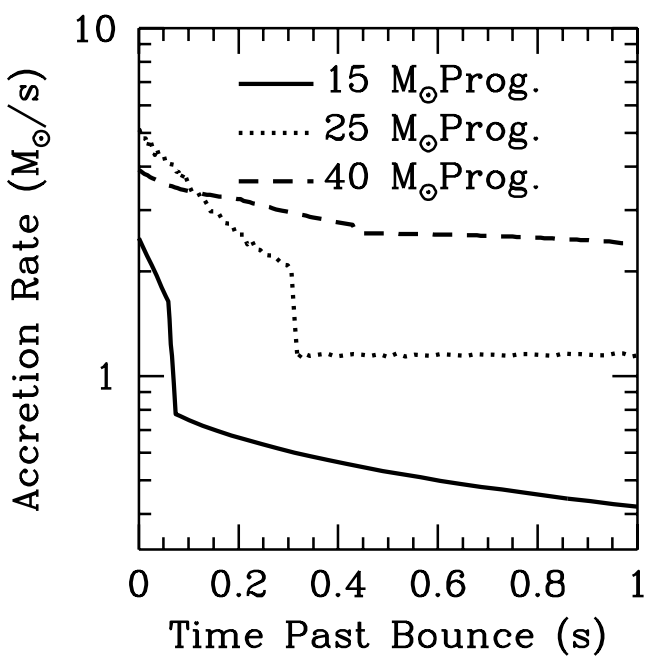

Figure 2: Accretion rates of different mass stars. The more massive stars have higher accretion rates and are, hence, harder to explode. Figure 1 from Fryer [97]. 
directly to a black hole without the formation of a proto neutron star and without the bounce associated with this formation [115, 217, 218]. But rotation can allow the formation of a proto black hole [115]. Because the binding energy of the star rises so quickly with stellar mass as the explosion energy goes down, the line dividing proto-neutron-star and black-hole formation can be determined fairly accurately (Figure 3). Of course, stellar winds will affect the stellar structure and also this fate (Figure 4).

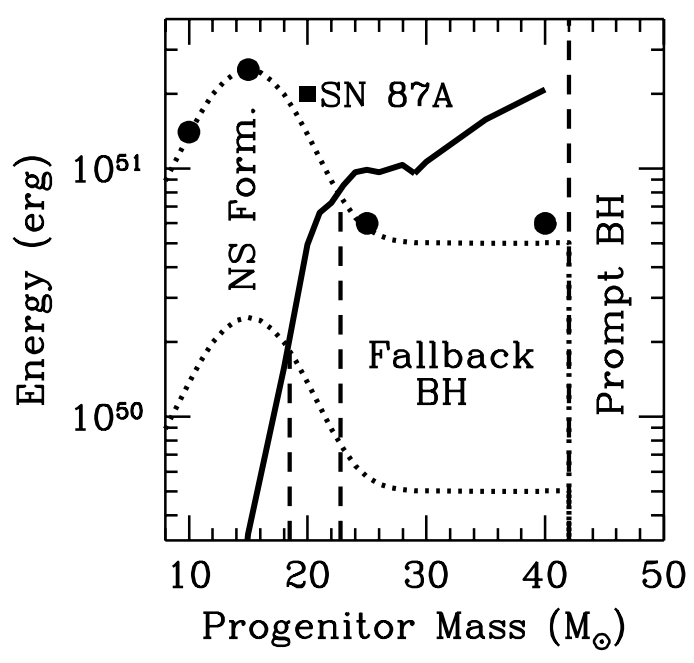

Figure 3: Supernova explosion energies (dotted lines) based on simulation (solid circles) and observation (solid dot of SN 1987A) as a function of progenitor mass. The binding energy of the envelop (material beyond the $3 M_{\odot}$ core) rises dramatically at $20 M_{\odot}$ (solid line). This fast rise with mass and the decline based on explosion simulations of the energies from the convective engine mean that even if the errors in these estimates are large, the likely dividing line between neutron star and black hole formation can be determined fairly accurately. Figure 1 of Fryer \& Kalogera [109].

One can take this one step further. If the energy of the explosion is stored in the convective region, one can estimate the maximum energy of the explosion based on the infalling accretion rate. The pressure in this convective region is [99]

$$
P(r)=\left[1 / 4 M_{\mathrm{NS}} G\left(S_{\mathrm{rad}} / S_{0}\right)^{-1}\left(1 / r-1 / r_{\text {shock }}\right)+P_{\text {shock }}^{1 / 4}\right]^{4} \mathrm{erg} \mathrm{cm}^{-3},
$$

where $M_{\mathrm{NS}}$ is the mass of the proto neutron star, $G$ is the gravitational constant, $S_{\mathrm{rad}}$ is the entropy in Boltzmann's constant per nucleon, $S_{0}=1.5 \times 10^{-11}$ and $r_{\text {shock }}$ and $P_{\text {shock }}$ are the radius and pressure of the accretion shock produced where the infalling stellar material hits the convective region. If we set $P_{\text {shock }}$, the ram pressure of the infalling material, $\equiv 1 / 2 \rho v_{\mathrm{ff}}^{2}$, where $\rho$ is the density of the infalling stellar material and $v_{\mathrm{ff}}$ is the free-fall velocity at the shock, we can estimate the maximum energy stored in the convective region (when it exceeds this value, the shock will move outward). This allows us to predict the explosion energy as a function of delay time in the explosion (Figure 5).

This picture does not change even if the standing accretion shock instability contributes to the convective instabilities. Many of the recent simulations have focused on the SASI and it is worth reviewing how it effects the basic convective picture of supernovae. This instability was originally discussed in the context of white-dwarf and neutron-star accretion in scenarios under the assumption that the accretion envelope was stable to Rayleigh-Taylor instabilities [144]. Asymmetries and strong Rayleigh-Taylor convection in actual models [101] coupled with the long 


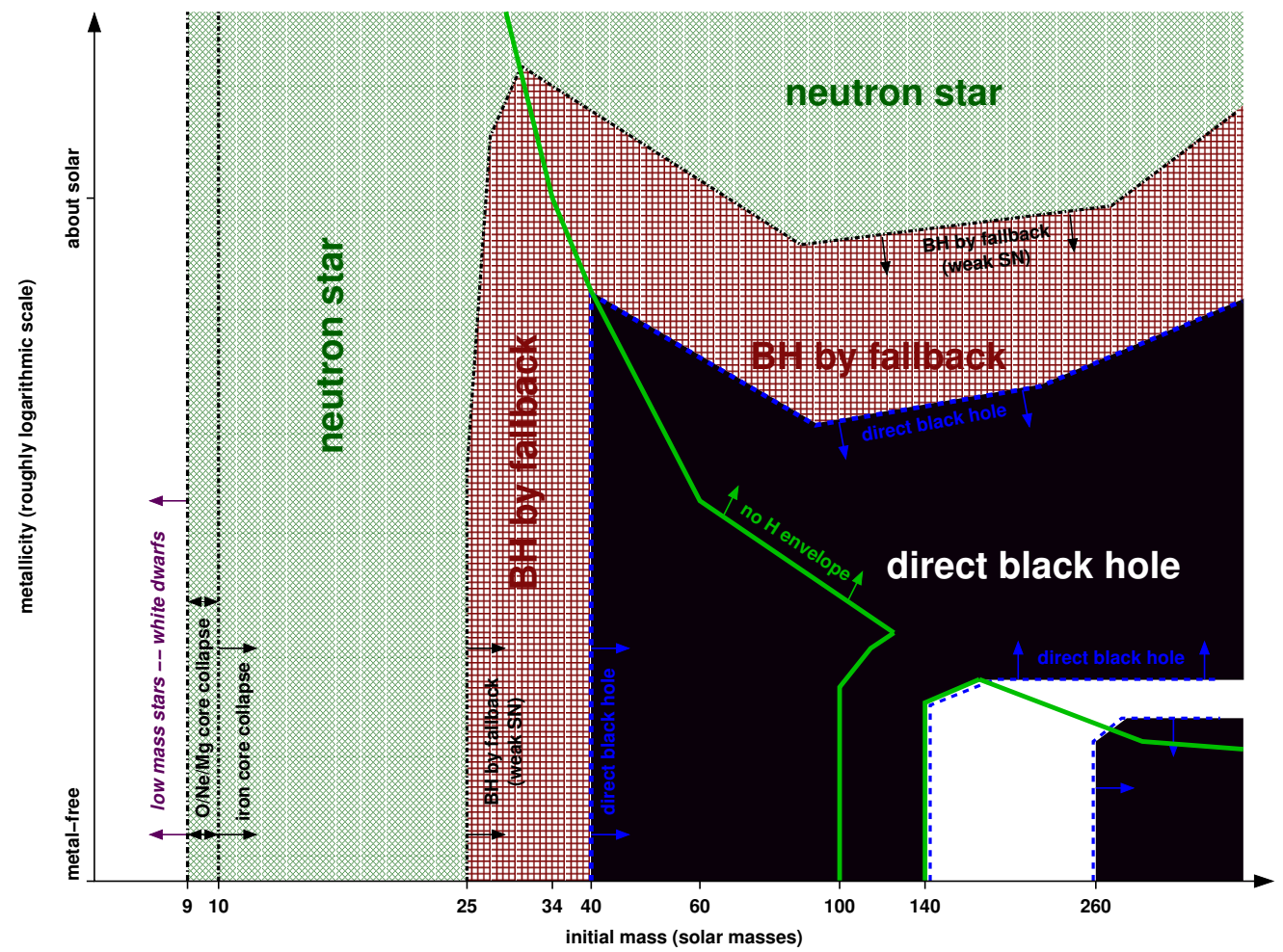

Figure 4: Fate of massive stars as a function of mass and metallicity. Figure 1 from Heger et al. [136]. 


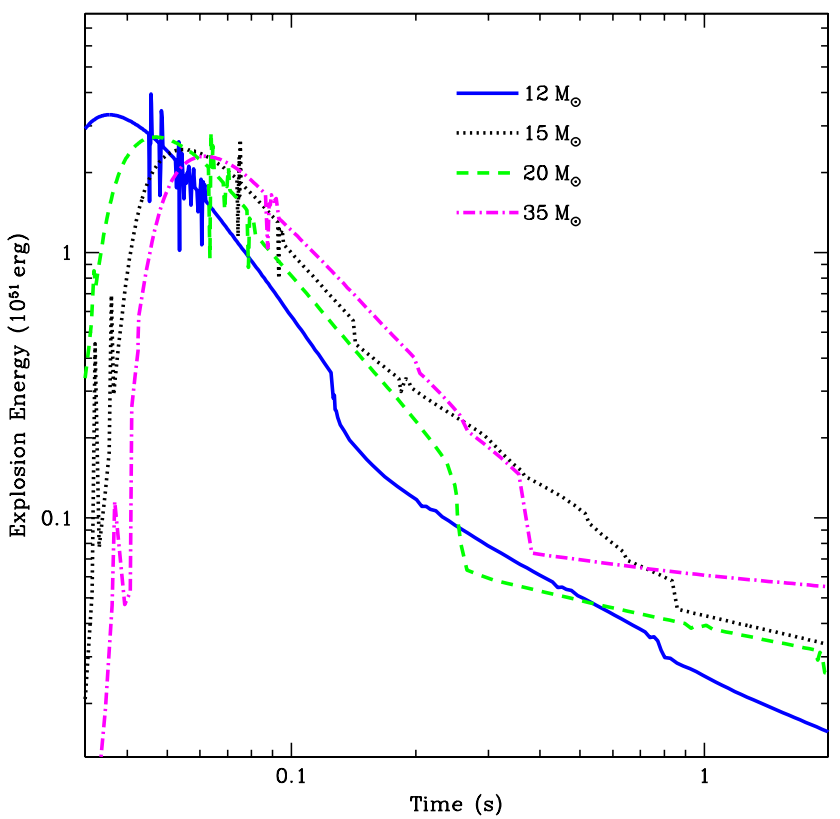

Figure 5: Explosion energy as a function of launch time of the supernova shock for four differently-massed stars assuming the energy is stored in the convective region. The energy is then limited by the ram pressure of the infalling stellar material. Note that it is difficult to make a strong explosion after a long delay. For more details, see [99] and Fryer et al. (in preparation). 
predicted growth times for the SASI $(\sim 3 \mathrm{~s}$ [144]) in neutron star accretion, led the accretion community to limit such instabilities to late times. Blondin et al. [21] introduced this instability into the core-collapse supernovae by setting up conditions that were stable to Rayleigh-Taylor instabilities to study the SASI. In this case, SASI dominates and, as Blondin et al. [21] found, can produce low-mode convection.

There are two dominant questions currently under discussion with regard to this instability. First, how dominant is SASI when entropy gradients do exist? Recall, the analytic estimates of the SASI were originally derived under the assumption that the envelope is Rayleigh-Taylor stable [144] and in neutron-star fallback; simulations argue that Rayleigh-Taylor instabilities dominate ${ }^{6}$. Lowmode convection is not a sure indicator of the SASI, as Rayleigh-Taylor instabilities also predict a growth toward low modes [139]). Second, is the SASI driven by an advective-acoustic instability or is it simply an acoustic instability? Blondin and collaborators, who first mentioned the possibility of advected vortices in the supernova context, later argued for an acoustic scenario [20]. Answering these question has spawned a great deal of both simulation and analytic theory work [38, 39, 82, 81, 94, 93, 92, 116, 195, 196, 229, 264, 263, 164, 336].

Our analysis of explosion energy holds regardless of the dominant instability or the cause of that instability if the assumption that the energy is stored in the convective region remains true. In such a case, it is difficult to construct a strong explosion from a mechanism that has a long delay. But other sources of energy exist. The neutron star itself can store energy in oscillations to add to the explosion energy [40, 39]. Fallback may also drive additional explosions [101, 100]. We will study both of these in more detail below. This has repercussions on the explosion engine and the resultant GW mechanisms.

Alternative mechanisms for the core-collapse supernova mechanism exist, most notably the magnetic-field mechanism $[175,299,2,3,6,35,61,62,265]$. The idea here is that magnetic fields strengthened in the collapse and subsequent convection (especially if the star is rotating rapidly) will drive an explosion. How the magnetic fields affect the GW signal will depend on how and when the magnetic field develops. For most magnetic-field generation schemes, the field grows after the bounce of the core, and signal from the collapse/bounce phase (and indeed some of the convective phase) from supernovae will be the same whether the mechanism is this alternate magnetic-field driven mechanism or the standard convective engine. For some magnetar models, the magnetic field develops after the launch of a weak, convectively-driven explosion [304]. In such a case, the GW signal will be identical to the convective-driven mechanism.

Finally, if the core collapses to a black hole and the infalling material has enough angular momentum to prevent its infall directly into the event horizon, explosions might be produced. An accretion disk forms and, either through the wind-up of magnetic fields or neutrino annihilation above the disk, an explosion may result. This type of explosion has been posited as the engine behind GRBs [219, 333]. The association of GRBs with supernova-like outbursts has argued strongly for a massive-star origin for at least long-duration GRBs [335, 117].

In this section, we review the various progenitors of core collapse, describing their physical properties, its occurrence rate, evolution, and likely GW emission mechanisms. Rather than follow the order of progenitor mass, we first discuss the fate of stars in the $12-20 M_{\odot}$ range, the likely progenitors for core-collapse supernovae. These are the most-studied gravitational-collapse systems. We then discuss the AIC of white dwarfs and the very similar collapse of stars in the $\sim 9-12 M_{\odot}$ range. We then move upwards in mass studying massive, very massive $\left(20-500 M_{\odot}\right)$ and supermassive $\left(>10^{5} M_{\odot}\right)$ stars.

6 In accretion, Rayleigh-Taylor instabilities occur due to entropy gradients in the envelope set up by accretion [101]. In a supernova engine, where the proto neutron star is hot, neutrino heating can also produce entropy gradients [140]. 


\subsection{Core-collapse supernovae}

Stars more massive than $\sim 10-12 M_{\odot}$ and less massive than $\sim 20 M_{\odot}$ are the primary candidates for what is considered the standard formation scenario behind core-collapse supernovae. For these stars, the current belief is that the collapse proceeds through bounce and proto neutron star formation. Convection, or perhaps an alternate mechanism like magnetic fields, then deposits enough energy above the proto neutron star to drive an explosion. Scientists presently think that this process is responsible for most type $\mathrm{Ib} / \mathrm{c}$ and type II supernovae ${ }^{7}$. This is the best studied, and probably best-understood, core-collapse scenario in astronomy.

\subsubsection{Core-collapse supernovae rate}

The rate of core-collapse supernovae can be determined by simply multiplying the star-formation rate times the fraction of stars in the $\sim 10-12$ and $\sim 20 M_{\odot}$ mass range. The uncertainties in such a calculation lie in determining the limits on either side of this range (determined by theory), the power in the initial mass function and the star formation rate (both beyond theory at this point). The latter two quantities, both set by observations, dominate the errors in this rate estimate.

Indeed, especially at high redshift, the supernova rate is better constrained by observations than the rate of star formation and it is often used to determine the star-formation history. The local rate of supernovae as a function of galaxy type has been studied $[43,193]$. The rate of supernovae in a Milky-Way-sized galaxy is roughly 1 per 100 y for type II and type Ib/c supernovae. It is believed that the rate has been decreasing since a redshift of roughly 1-1.5 (Figure 6).

\subsubsection{Core-collapse evolution}

The evolution of a core-collapse supernova passes through a number of phases: collapse and bounce, post-bounce and convection and neutron-star cooling. The runaway collapse of the core continues until the core reaches nuclear densities. At this point, nuclear forces and neutron degeneracy pressure sharply increase the core pressure, causing the core to bounce and sending a shock wave throughout the star. The nature of the bounce depends upon the equation of state: a stiffer equation of state causes the star to bounce more quickly but also more weakly than a softer equation of state. Within the current uncertainties, the bounce still occurs at an enclosed mass (mass zone measured from the center of the star) of $0.55 \pm 0.2 M_{\odot}$. By the time the bounce shock stalls, the mass of the core is closer to $0.9 \pm 0.2 M_{\odot}$. Including the effects of general relativity acts to effectively soften the equation of state. Rotation can cause the bounce to occur at lower densities (as centrifugal support contributes to the bounce), but for the current fastest-rotating cores produced in stellar models, this effect is less than $20 \%$ [113, 69].

As the bounce shock moves outward, it becomes optically thin to neutrinos and the shock loses its internal energy. For these "standard" core-collapse explosions, the shock then does not have enough energy to throw off the infalling star and the shock stalls. But it leaves behind an entropy profile that is convectively unstable. Furthermore, heating from the hot core also drives convection $^{8}$. If the convective region can develop enough energy to blow off the infalling star, a successful supernova explosion (Figure 7) results. Especially for explosions with long delays

\footnotetext{
7 These different supernova types are thought to differ primarily based on the envelope left on the star at collapse, not based on a different collapse mechanism: type II supernovae still have a hydrogen envelope, whereas type Ib/c SNe have lost their hydrogen, and for the type Ic SNe, most of its helium, envelopes.

8 Fryer \& Young [116] have pointed out that current simulations are likely to be under-resolved and predict less vigorous convection than what should happen in nature: see also [41, 70]. In general, models of convective instabilities suggest that high resolution (above $512^{3}$ zones) across a convective cell is required to resolve convection accurately (e.g., [243]). If such constraints from the turbulence community are correct, the current grid-based simulations in core collapse (e.g., Marek \& Janka [195]) remain under-resolved.
} 

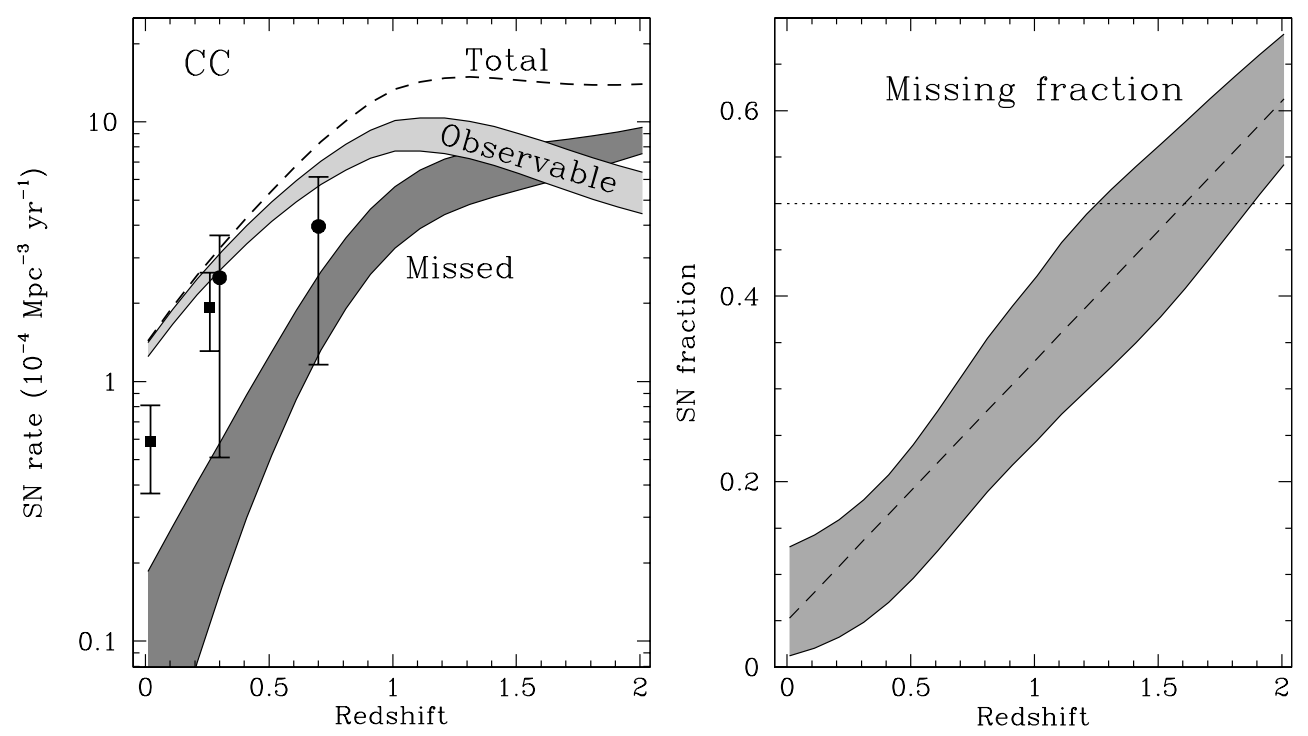

Figure 6: Left-hand panel: Evolution of the core-collapse supernova rate with redshift. The dashed line shows the intrinsic total supernova rate derived from the star formation density. The supernova rate that can be recovered by optical searches is shown in light gray, and is compared with the data points from Cappellaro et al. [44] (squares) and Dahlen et al. [59] (circles). Right-hand panel: Fraction of CC SNe that are not present in the optical and near-IR searches as a function of redshift. (Figure 2 of Mannucci et al. [193]; used with permission.) 

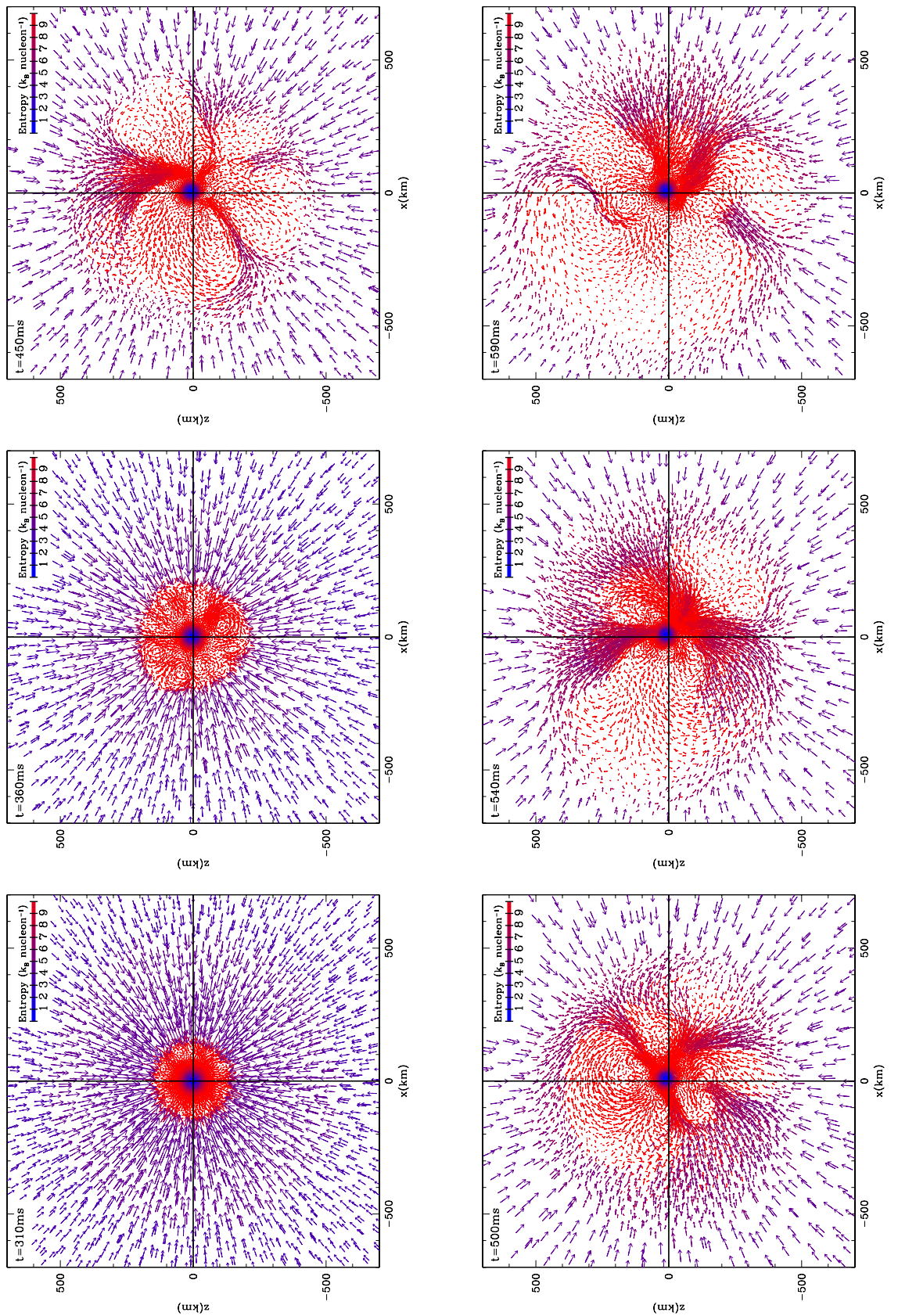

Figure 7: Six snapshots in time of the convection in the collapse of a $23 M_{\odot}$ star. The plot displays slices of the data in the $\mathrm{x}-\mathrm{z}$ plane. The vectors denote direction and magnitude of the particle motion. The colors denote entropy. The convection is far from symmetric, but we do not get the single-sided downflows seen in many recent 2-dimensional calculations. Figure 4 of [116]. 
(greater than a few hundred ms), the SASI may play a strong role in the convection (see the review at the beginning of this section).
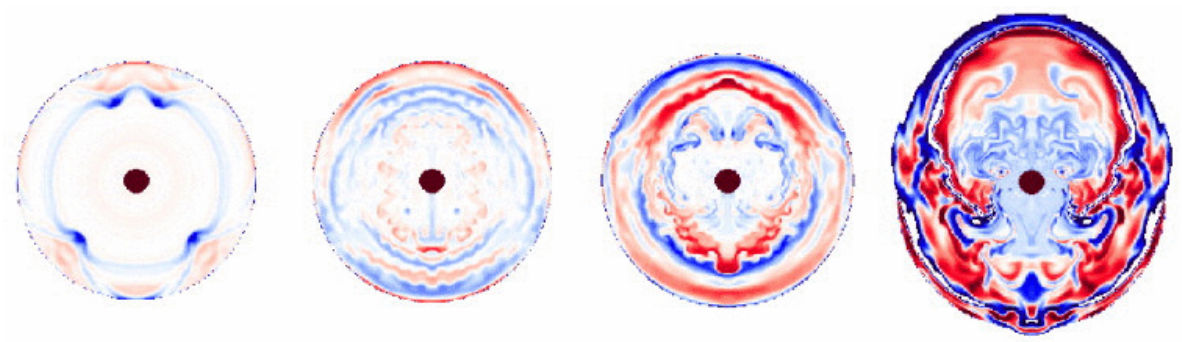

Figure 8: Images of the gas entropy (red is higher than the equilibrium value, blue is lower) illustrate the instability of a spherical standing accretion shock. This model has $\gamma=4 / 3$ and is perturbed by placing overdense rings into the infalling preshock gas. The shock is kept stalled by using a cooling function. Note that with the scaling for a realistic supernova model, the last image on the right corresponds to $300 \mathrm{~ms}$. These simulations are axisymmetric, forcing a reflection symmetry about the vertical axis. (Figure 6 of [21]; used with permission.)

In any event, both instabilities, if driven to late times, will naturally produce explosions with asymmetries that may explain the observational evidence for asphericities in the core-collapse explosion: pulsar velocities, mixing, polarization (Figure 8). It may also produce stronger gravitational wave signals than the higher-mode neutrino-driven convection. Fryer [99] has argued that the long delays required for SASI to dominate leads to low-energy explosions that do not eject much ${ }^{56} \mathrm{Ni}$ and produce dim supernovae (Figure 5). If true, any extremely delayed (more than $400 \mathrm{~ms}$ ) explosion mechanism does not match supernova observations and cannot be the dominant fate of stellar collapse (although given the rough observed limits on the supernova rate and the difficulty in observing dim supernovae, we cannot constrain the importance of this mechanism with any certainty).

As the proto neutron star cools, it may also develop strong convection and this could be another source of GWs [160, 34]. But the extent of this convection ranges from growing throughout the entire proto neutron star [160], to select regions in the star [60], to small regions in the star $[140,112,113,116]$. Only when the convection is in the entire proto neutron star [160] is it considered to play a large role in the supernova explosion. Although a small contribution to the GW signal, this convection may not be negligible in calculating the full GW signal from core collapse [228].

Another possible source of GWs is the oscillations in the proto neutron star [40]. But, like the strong proto-neutron-star convection, there are arguments against such strong oscillations [116, 85, 343, 195, 327].

In strongly magnetized neutron stars, when neutrino oscillations into sterile neutrinos in the core occur, we also expect asymmetries in the neutrino emission to lead to asymmetries in the neutrinos escaping the core, producing GWs [170, 120].

But, by far, the most studied source of GWs from proto neutron stars are the rotationallyinduced bar-mode instabilities. All of these can contribute to the outflows from the cooling neutron star. If magnetic fields develop or there is a high mass-infall rate [305], these outflows can add significantly to the explosion energy and at least one supernovae seems to have experienced such a secondary explosion [191]. 


\subsubsection{Core collapse: Sources of gravitational wave emission}

In Section 2, we reviewed the wide variety of mechanisms that lead to GW emission, i.e., time varying quadrupole moments in the matter or the radiation. The complex dynamics and physics within the evolution of a standard core-collapse supernova allow for a variety of scenarios for GW emission at different phases in the collapse.

Bounce: At core bounce, when the infalling material reaches nuclear densities and the collapse halts, the matter reaches its peak acceleration. If the collapse phase is asymmetric, either by asymmetries in the stellar structure or through rotation, this phase can lead to the strongest GW emission. The primary source of this emission is the rapidly changing quadrupole moment in the matter as the asymmetries evolve (Section 4.1). But the asymmetries in the bounce also produce the initial asymmetry in the neutrino emission and this asymmetry may lead to a strong GW signal as well (Section 4.4).

Post-bounce and Convection: The convection above the proto neutron star can also develop strong asymmetries as the convective cells merge into low-mode convection. This can lead to rapidly varying quadrupole moments in both the matter (Section 4.2) and the core (Section 4.4).

In the Neutron Star: Convection in the cooling neutron star could produce strong GW emission. So can asymmetric neutrino emission produced by neutrino oscillations to stellar neutrinos in the core ${ }^{9}$. Pulsations in the newly formed proto neutron star may also produce a strong GW signal [85, 229]. But the most-studied GW source arises from bar-mode instabilities, in part because, if they develop, they may produce a GW signal that rivals both the bounce and convective GW signals.

\footnotetext{
${ }^{9}$ Neutrino emission is tied to electron spin, which is made anisotropic by strong magnetic fields [170, 120].
} 


\subsection{Accretion-induced collapse}

Stars with masses in the range $\sim 1-8 M_{\odot}$, through burning flashes in an Asymptotic Giant Branch (AGB) and post-AGB phase, ultimately lose their envelopes, leaving behind their Carbon/Oxygen (CO) cores (now CO White Dwarfs) and a planetary nebula (for a review of AGB evolution, see Herwig [141] or recent papers [286, 287, 241]). If it were not for the fact that many of these newly formed white dwarfs are in binary systems, such objects would be unimportant for stellar collapse. But there is growing evidence that perhaps most planetary nebulae are formed in binaries [200]. If a white dwarf accretes enough matter through binary mass transfer, its mass can approach the Chandrasekhar limit, causing it to collapse and produce a supernova explosion.

Whether this supernova explosion is produced by the burning of this CO white dwarf into nickel (type Ia Supernova) or by the "Accretion Induced Collapse" (AIC) of the white dwarf down to a neutron star (making these objects relevant to this review) depends upon the accretion rate onto this white dwarf. Nomoto \& Kondo [224] summarized the fate of an accreting white dwarf presented as a function of its initial mass and the accretion rate. When material accretes, it ignites. And the fate of the accreting white dwarf both depends on this ignition process (does the accreting material explode as a nova, ejecting matter, or does it burn less explosively, accreting onto the white dwarf) and also the ultimate ignition of the accreting white dwarf itself. Heat inflow ignites white dwarfs with masses below about $1.2 M_{\odot}$ at low densities, causing thermonuclear explosions. AICs are only produced in stars with masses below $\sim 8 M_{\odot}$ in one of two categories:

- high white-dwarf mass: If the white dwarf is massive (above $\sim 1.2 M_{\odot}$ ), the heat inflow to the core is minimal and the white dwarf heats up primarily due to adiabatic compression. In this case, by the time the white dwarf ignites, it is very dense and the URCA process can cause significant cooling, causing the star to collapse instead of explode.

- high accretion rates: If the accretion rate is very high, the accretion process will ignite carbon at the edge of the white dwarf, burning it into an Oxygen/Neon (ONe) white dwarf. In this case, Urca processes provide a cooling mechanism causing the star to collapse as it reaches the Chandrasekhar limit (see [127] for review and counterexamples).

At this time, stellar modelers do not expect that Nature produces that many $\mathrm{CO}$ white dwarfs with initial masses above $1.2 M_{\odot}[287,241]$. The most massive white dwarfs are probably formed as $\mathrm{ONe}$ white dwarfs from stars above $8 M_{\odot}$ - see Section 3.3. So this class of AIC formation is likely to be rare. But high accretion rates are expected in a very important, and perhaps common, event: the merger of two white dwarfs (generally a He or CO white dwarf merging with a CO white dwarf). Most current simulations predict that this merger process is very rapid, arguing that the more-massive white dwarf will tidally disrupt and accrete its companion in a very short time. Such a rapid process suggests high accretion rates, arguing that most mergers produce AICs. However, there are a number of uncertainties in such calculations and no other mechanism produces a sufficiently high rate to explain the occurrence of type Ia supernovae, so we cannot rule out that these mergers produce thermonuclear supernovae instead of AICs.

\subsubsection{AIC rates}

Thus far, no outburst from the AIC of a white dwarf has been observed. Given that the outburst is expected to be very dim because the shock heating is negligible and the predicted ${ }^{56} \mathrm{Ni}$ yields are all low $\left(<0.05 M_{\odot}[102,161,61]\right)$, the lack of observed AICs does not place firm upper limits on the AIC rate.

Theoretical estimates of the rate of AICs are also quite uncertain. If we accept the conclusions of Nomoto \& Kondo [224], the AIC rate may be well above the type Ia supernova rate $\left(\sim 10^{-2} \mathrm{y}^{-1}\right.$ in a Milky-Way-sized galaxy). This result depends upon a number of assumptions in the accretion 
process in these binary systems and the true rate of AICs could be many orders of magnitude lower than this value. Studies of binary mass transfer [198, 199, 16, 270, 126, 203, 72, 342] and white dwarf accretion [91] are both becoming more accurate. As they are coupled with stellar evolution models of these systems, our understanding of the rate of AICs should become more accurate as well.

Alternatively, one can use observed features of AIC explosions to constrain the AIC rate. Fryer [102] argued that the neutron-rich ejecta from an AIC limits their rate to $\sim 10^{-4} \mathrm{y}^{-1}$. More recent results, which eject a smaller fraction of neutron-rich material, may loosen this limit by 1 order of magnitude [161, 61].

\subsubsection{Evolution of AIC}

As a white dwarf accretes material up to the Chandrasekhar limit, it will begin to compress. As it does, it heats up and, in principle, can ignite explosive burning. But the URCA process cooling (electron capture followed by a beta decay, which leads to the emission of two neutrinos, which escape and cool the system, see Couch \& Arnett [58] for a summary) on nuclei can allow the white dwarf to cool enough that the burning does not generate enough energy to disrupt the white dwarf. When the white dwarf becomes sufficiently dense, the material is dissociated and the collapse proceeds in a very similar manner to that of a "standard" core-collapse supernova. The core proceeds through a runaway collapse that ends when nuclear forces and neutron degeneracy pressure halt the collapse, causing a "bounce". Especially in the case of a white dwarf/white dwarf merger, the accretion phase just prior to collapse is likely to spin up the core and these are almost certainly the most rapidly-rotating core-collapse models.

The structure of AIC cores is very similar to the structure of low-mass stars, and a large set of simulations of both have been conducted in the past few decades [142, 11, 334, 102, 63, 61]. The latest simulations [63, 61] use progenitors including rotation [341, 339]. The shock stalls in AICs, just as in standard core-collapse supernovae, but there is no stellar envelope with which to prevent further accretion and the explosion is quickly revived (hence there will be little convective overturn). The cooling neutron star could well develop instabilities (both to convection and to bar modes). If magnetic fields develop, additional outflows can occur.

\subsubsection{AIC: Sources of gravitational wave emission}

Due to the lack of convection, AICs may only have a subset of the GW sources that we see in core-collapse supernovae. However, AICs have the potential to be much faster rotating, which may make them strong GW sources.

Bounce: The potentially high-rotation in AICs mean that the bounce signal in these systems could be quite large. The primary source of this emission is the rapidly changing quadrupole moment in the matter as the asymmetries evolve (Section 4.1), although we cannot rule out a strong signal from asymmetric neutrino emission (Section 4.4).

In the Neutron Star: Convection in the cooling neutron star could produce GW emission. But more likely, due to the strong rotation, bar-mode instabilities can become important in these collapsed systems (Section 4.3). 


\subsection{Electron-capture supernovae and core-collapse supernovae below 10 - 12 solar masses: Low mass stars}

Stars below about 10-12 $M_{\odot}([286,287,241]$, Poelarends et al., in preparation $)$ have very diffuse envelopes surrounding their cores when they collapse. The lowest mass stars in this class lie on the border between white dwarf (and planetary nebula) formation and the formation of an iron core (see Herwig [141] for a review). These stars tend to form ONe white dwarfs, ejecting most of their envelope. They are supported by electron degeneracy pressure and electron capture causes them to collapse down to neutron stars. Their resultant explosions are termed Electron Capture Supernovae [152]. The more massive stars in this class do form iron cores, but the envelopes surrounding these core have low densities (when compared to normal supernovae progenitors) at the time of collapse.

\subsubsection{Rate of supernovae from low-mass stars}

The rate of core collapse from low mass stars has the same uncertainties of star formation rate and initial mass function of stars that made it difficult to estimate the normal supernova rate. But it also has an additional uncertainty: understanding the lower limit at which these collapses occur [197, 286, 287, 241]. This lower limit marks the dividing line between white dwarf and neutron star formation and the explosive ejection of the star's envelope (part of the white dwarf formation process) has proven very difficult for traditional stellar evolution codes to model. The actual position of this lower limit has been a matter of debate for over three decades (see Iben \& Renzini [152] for a review). The metallicity dependence on this limit is even more controversial. Heger et al. [136] argued that the metallicity dependence was negligible and that the lower limit was roughly at $9 M_{\odot}$ at all metallicities. Poelarends et al. [241] have studied this matter in more detail and have a range of solutions for the metallicity dependence. For neutron stars forming through electron capture supernovae, their "preferred" model predicts that the limit drops from $9 M_{\odot}$ at solar metallicity down to $6.3 M_{\odot}$ at $z_{\text {metal }}=10^{-3} z_{\odot}$. This means that for most initial mass functions, these low mass core-collapse progenitors dominate core-collapse at low metallicities.

Unfortunately, these low-mass supernovae do not produce very much radioactive $\mathrm{Ni}$, the power source for supernova light-curves. Although their envelopes are likely to be denser than the material surrounding AICs, the tenuous envelopes certainly affect the explosion and may limit the shockpowered light-curve. But surveys are now catching these supernovae [288]. These surveys will ultimately place reasonable estimates on the rate of these supernovae. Based on current estimates of the mass limits and the initial mass function in this mass range, we expect the rate of these supernovae to be roughly equal to that of the core-collapse supernova rate.

\subsubsection{Low-mass star collapse: Evolution}

The structure of the core of low-mass stars is very similar to the structure of AIC cores, and a large set of simulations of both have been conducted in the past few decades [142, 11, 334, 102, 63, 61]. The core collapses and bounces, but the envelope is not massive enough to prevent a quick revival. A convective region is unlikely to develop above the proto neutron star. But these stars are probably not rotating as fast as most AICs, and their subsequent GW emission is likely to be weaker (e.g., smaller chance of bar-mode instabilities, etc.).

\subsubsection{Low-mass star collapse: Gravitational wave emission mechanisms}

The GW emission mechanisms at bounce and in the neutron star are the same as AICs but the likelihood of the development of instabilities (and the strength of the GW emission from these instabilities) will be affected by slower rotation. 


\subsection{Core-collapse supernovae from stars above 20 solar masses: Massive star collapse}

In the absence of winds, as the stellar mass approaches $20 M_{\odot}$, the explosion energy predicted by engine models decreases while the binding energy of these stars increases (recall Figure 3). As this happens, more and more material from the stellar mantle falls back onto the newly formed neutron star. Roughly at $20 M_{\odot}$, so much material falls back that the mass of the compact remnant exceeds the maximum neutron star mass and ultimately collapses to form a black hole. However, note that Heger et al. [136] argue that, at solar metalliciety, many massive stars lose so much mass in their winds that they will form neutron stars, not black holes (recall Figure 4).

The formation of a black hole opens up an entirely new set of physics in the stellar collapse. Material continues to accrete onto the black hole after its formation. The angular momentum of material in a star tends to increase with increasing radius of the star (Figure 9). For rapidly-rotating stars, the angular momentum of the accreting material will ultimately be sufficiently high to hang up in a disk. Astronomers have argued that energy generation in this accretion disk (either through magnetic field generation or neutrino emission) may drive Gamma-Ray Bursts [242, 189, 244, 250]. As we shall see below, these black hole accretion disks also open up new possibilities for GW emission.

\subsubsection{Massive star collapse: Evolution}

The evolution of a massive star collapse begins identically to lesser mass stars. In general, it is likely that the core bounces just as in normal core-collapse supernovae [90, 97, 296, 295]. ${ }^{10}$ The bounce shock stalls as in normal supernovae. The primary exception are the low metallicity $\left(<10^{-4} Z_{\odot}\right)$ stars in the $140-260 M_{\odot}$ range that produce pair-instability supernovae [114]. These stars explode completely leaving no remnant behind. We will ignore these stars in the rest of our discussion.

These massive stars are much less likely to revive the stalled shock. Although the region above the proto neutron star is convective, the convective engine is unable to quickly drive an explosion (recall Section 3.1). In such cases, low-mode instabilities (e.g., SASI, see Section 3.1.2) are more likely to dominate the convection. If the shock is revived at all, the explosion is weaker than normal supernovae. Considerable material begins to fall back within $1-2 \mathrm{~s}$ of the explosion with accretion rates nearly at $1 M_{\odot} \mathrm{s}^{-1}[190,347,100]$. This accretion may lead to additional convection and possible further outbursts $[105,100]$. With both the SASI and the accretion convection, these stars are likely to have more asymmetric convection than normal core-collapse supernovae (beneficial for GW emission).

Ultimately, the mass accretion causes the proto neutron star to collapse down to a black hole [55, 54]. After the collapse, a disk forms around the black hole if the star's angular momentum is sufficiently high. Magnetic dynamos in the disk, or neutrino emission, might lead to an additional explosion. Indeed, this is the mechanism behind the collapsar GRB [219, 333].

Massive stars might avoid the collapse to a black hole if strong magnetar-like fields can be produced in the dense environment produced when the above engine fails [35, 175, 2, 3, 6, 329].

\subsubsection{Rates of massive star collapse}

The rate of massive star collapse can be determined by the same method used to determine the normal supernova rate: multiplying the star formation rate times the fraction of stars above $\sim 20 M_{\odot}$. The uncertainties in such a calculation lie in determining the lower limit for black hole

\footnotetext{
10 The possible exception is extremely massive stars above $\sim 300 M_{\odot}$. Fryer et al. [114, 217, 218] found that such stars may have enough entropy and angular momentum to alter the "bounce" density (and mass) considerably. These stars form proto black holes and collapse to a black hole shortly after the initial bounce with no supernova explosion whatsoever.
} 

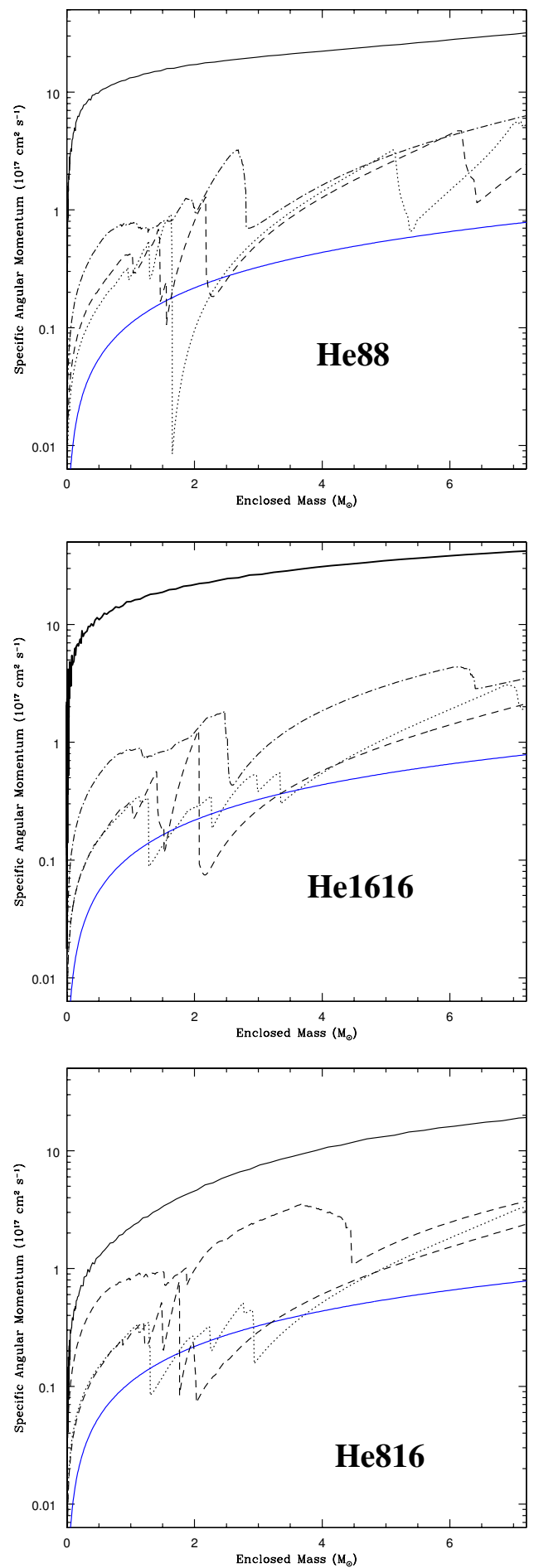

Figure 9: Mean angular momenta vs. mass for both merged stars just after the merger and at collapse. The thick solid line at the top of the graph is the angular momentum just after merger. Dotted lines correspond to different mappings of the 3-dimensional merger calculation into the 1-dimensional stellar evolution code. We also plotted the angular momentum for material at the innermost stable circular orbit for a non-rotating black hole vs. mass (thin solid line). Figure 5 of [104]. 
formation (determined by theory, and bolstered by observations), the power in the initial mass function and the star formation rate (both beyond theory at this point). As with core collapse, the latter two quantities dominate the errors in this rate estimate. Fryer \& Kalogera [109] argue that between $10-40 \%$ of all massive stars above $10 M_{\odot}$ (those that are likely to form bright corecollapse supernovae and contribute to the observed supernova rate) form black holes. This answer depends primarily on their prescription for winds and the initial mass function. The fraction is likely to be lower at low-redshift but increase with increasing redshift as winds remove less mass from the star, allowing more stars to form black holes.

Unfortunately, there are no direct observational estimates for the black-hole-formation rate. If we assume this collapse is the primary scenario for the production of long-duration GRBs we can estimate a lower limit for the black-hole-formation rate (and estimate the formation rate of fast-rotating systems). This value is roughly $0.1-0.01 \%$ the total core-collapse supernova rate.

\subsubsection{Gravitational waves from massive stars}

For most massive stars, the GW signal will be similar to normal core-collapse supernovae. Massive stars above $300 M_{\odot}$ are likely to have much stronger signals than normal core-collapse supernovae, but they will only occur at high redshift.

Bounce: For the lower-mass range of these stars, the signal is unlikely to be demonstrably different than normal core-collapse supernovae.

Post-Bounce and Convection: Because the convective phase in these massive stars persists longer than normal supernovae, it can develop strong low-mode activity. Such convective cycles produce the strongest GWs, so we might expect stronger GWs from this convective phase for massive stars than for any other stellar collapse scenario. Fryer et al. [115] also found that bar instabilities could develop in $\sim 300 M_{\odot}$ stars.

Black Hole Formation: The initial black-hole formation and the subsequent accretion leads to a perturbed geometry of the black hole, initially distorting it. This distortion causes the hole to "ring" in distinct harmonics as gravitational radiation removes the perturbation and the black hole settles into a stationary Kerr state.

Disk Fragmentation: Depending upon the angular momentum profile, the mass in the disk can be large enough that self-gravity can drive instabilities and induce fragmentation.

\subsection{Supermassive stars}

There is a large body of observational evidence that supermassive black holes (SMBHs, $M \gtrsim$ $10^{6} M_{\odot}$ ) exist in the centers of many, if not most galaxies (see, e.g., the reviews of Rees [246] and Macchetto [188]). The masses of SMBHs in the centers of more than 45 galaxies have been estimated from observations [83] and there are more than 30 galaxies in which the presence of a SMBH has been confirmed [163].

\subsubsection{Supermassive stars: Evolution}

One of the possible formation mechanisms for SMBHs involves the gravitational collapse of SMSs. The timescale for this formation channel is short enough to account for the presence of SMBHs at redshifts $z>6$ [155]. SMSs may contract directly out of the primordial gas, if radiation and/or magnetic-field pressure prevent fragmentation [131, 75, 130, 186, 29, 1, 348]. Alternatively, they may build up from fragments of stellar collisions in clusters [262, 14]. Supermassive stars are radiation dominated, isentropic and convective $[276,345,186]$. Thus, they are well represented by an $n=3$ polytrope. If the star's mass exceeds $10^{6} M_{\odot}$, nuclear burning and electron/positron annihilation are not important. 
After formation, an SMS will evolve through a phase of quasi-stationary cooling and contraction. If the SMS is rotating when it forms, conservation of angular momentum requires that it spins up as it contracts. There are two possible evolutionary regimes for a cooling SMS. The path taken by an SMS depends on the strength of its viscosity and magnetic fields and on the nature of its angular momentum distribution.

In the first regime, viscosity or magnetic fields are strong enough to enforce uniform rotation throughout the star as it contracts. Baumgarte and Shapiro [12] have studied the evolution of a uniformly-rotating SMS up to the onset of relativistic instability. They demonstrated that a uniformly-rotating, cooling SMS will eventually spin up to its mass shedding limit. The mass shedding limit is encountered when matter at the star's equator rotates with the Keplerian velocity. The limit can be represented as $\beta_{\text {shed }}=(T /|W|)_{\text {shed }}$. In this case, $\beta_{\text {shed }}=9 \times 10^{-3}$. The star will then evolve along a mass-shedding sequence, losing both mass and angular momentum. It will eventually contract to the onset of relativistic instability [151, 49, 50, 276, 155, 185].

The SMS formation scenario for SMBHs is just one of many. It fits into a broad class of scenarios invoking the collapse of supermassive objects formed in halos of dense gas, e.g., [15]. Although the structure used in many of these SMS calculations may not be appropriate for this broad class of supermassive objects, many of the basic features studied in these SMS simulations will persist.

Baumgarte and Shapiro used both a second-order, post-Newtonian approximation and a fully general-relativistic numerical code to determine that the onset of relativistic instability occurs at a ratio of $R / M \sim 450$, where $R$ is the star's radius and $G=c=1$ in the remainder of this section. Note that a second-order, post-Newtonian approximation was needed because rotation stabilizes the destabilizing role of non-linear gravity at the first post-Newtonian level. If the mass of the star exceeds $10^{6} M_{\odot}$, the star will then collapse and possibly form a SMBH. If the star is less massive, nuclear reactions may lead to explosion instead of collapse.

The major result of Baumgarte and Shapiro's work is that the universal values of the following ratios exist for the critical configuration at the onset of relativistic instability: $T /|W|, R / M$, and $J / M^{2}$. These ratios are completely independent of the mass of the star or its prior evolution. Because uniformly-rotating SMSs will begin to collapse from a universal configuration, the subsequent collapse and the resulting gravitational waveform will be unique.

In the opposite evolutionary regime, neither viscosity nor magnetic fields are strong enough to enforce uniform rotation throughout the cooling SMS as it contracts. In this case, it has been shown that the angular momentum distribution is conserved on cylinders during contraction [22]. Because viscosity and magnetic fields are weak, there is no means of redistributing angular momentum in the star. So, even if the star starts out rotating uniformly, it cannot remain so.

The star will then rotate differentially as it cools and contracts. In this case, the subsequent evolution depends on the star's initial angular momentum distribution, which is largely unknown. One possible outcome is that the star will spin up to mass-shedding (at a different value of $\beta_{\text {shed }}$ than a uniformly-rotating star) and then follow an evolutionary path that may be similar to that described by Baumgarte and Shapiro [12]. The alternative outcome is that the star will encounter the dynamical bar instability prior to reaching the mass-shedding limit. New and Shapiro [222, 223] have demonstrated that a bar-mode phase is likely to be encountered by differentially-rotating SMSs with a wide range of initial angular-momentum distributions. This mode will transport mass and angular momentum outward and thus may hasten the onset of collapse.

\subsubsection{Rates of supermassive stars}

An estimate of the rate of the collapse of SMSs can be derived from the quasar luminosity function. Haehnelt [129] has used the quasar luminosity function to compute the rate of GW bursts from SMBHs, assuming that each quasar emits one such burst during its lifetime (and that each quasar 
is a supermassive black hole). If it is assumed that each of these bursts is due to the formation of a supermassive black hole via the collapse of an SMS, then Haehnelt's rate estimates can be used as estimates of the rate of SMS collapse. This rate is likely an overestimate of the SMS collapse rate because many SMBHs may have been formed via merger. Haehnelt predicts that the integrated event rate through redshift $z=4.5$ ranges from $\sim 10^{-6} \mathrm{yr}^{-1}$ for $M=10^{8} M_{\odot}$ objects to $\sim 1 \mathrm{yr}^{-1}$ for $M=10^{6} M_{\odot}$ objects. Thus, as in the case of Population III stars, a reasonable occurrence rate can be determined for an observation (luminosity) distance of $50 \mathrm{Gpc}$.

\subsubsection{Gravitational-wave emission mechanisms of supermassive stars}

The GW emission mechanisms related to the collapse of SMSs are a subset of those discussed in the sections on AIC, SNe/collapsars, and Population III stellar collapse.

Bounce: Like very massive stars [97], it is likely that these SMSs will first form a proto black hole before collapsing to form a black hole. If so, they can emit GWs from the bounce that occurs when the proto black hole is formed. Because of the mass of the SMS, an aspherical collapse can produce a much stronger signal than any normal supernovae.

Post-Bounce: GWs may be emitted due to global rotational and fragmentation instabilities that may arise during the collapse/explosion and in the collapsed remnant (prior to black-hole formation).

Black Hole: Ringing in the black hole as it forms and accretes matter may also drive a strong signal. 


\section{Gravitational Wave Emission Mechanisms}

The collapse of the progenitors of core-collapse supernovae has been investigated as a source of gravitational radiation for more than three decades. In an early study published in 1971, Ruffini and Wheeler [251] identified mechanisms related to core collapse that could produce GWs and provided order-of-magnitude estimates of the characteristics of such emission.

Quantitative computations of GW emission during the infall phase of collapse were performed by Thuan and Ostriker [311] and Epstein and Wagoner [78, 77], who simulated the collapse of oblate dust spheroids. Thuan and Ostriker used Newtonian gravity and computed the emitted radiation in the quadrupole approximation. Epstein and Wagoner discovered that post-Newtonian effects prolonged the collapse and thus lowered the GW luminosity. Subsequently, Novikov [225] and Shapiro and Saenz [273, 253] included internal pressure in their collapse simulations and were thus able to examine the GWs emitted as collapsing cores bounced at nuclear densities. The quadrupole GWs from the ringdown of the collapse remnant were initially investigated by the perturbation study of Turner and Wagoner [318] and later by Saenz and Shapiro [254, 255].

Müller [204] calculated the quadrupole GW emission from 2D axisymmetric collapse based on the Newtonian simulations of Müller and Hillebrandt [207] (these simulations used a realistic equation of state and included differential rotation). He found that differential rotation enhanced the efficiency of the GW emission.

Stark and Piran [291, 238] were the first to compute the GW emission from fully-relativistic collapse simulations, using the ground-breaking formalism of Bardeen and Piran [10]. They followed the (pressure-cut induced) collapse of rotating polytropes in 2D. Their work focused in part on the conditions for black-hole formation and the nature of the resulting ringdown waveform, which they found could be described by the quasi-normal modes of a rotating black hole. In each of their simulations, less than $1 \%$ of the gravitational mass was converted to GW energy.

Seidel and collaborators also studied the effects of general relativity on GW emission during collapse and bounce [271, 272]. They employed a perturbative approach, valid only in the slowlyrotating regime.

The gravitational radiation from non-axisymmetric collapse was investigated by Detweiler and Lindblom, who used a sequence of non-axisymmetric ellipsoids to represent the collapse evolution [64]. They found that the radiation from their analysis of non-axisymmetric collapse was emitted over a more narrow range of frequency than in previous studies of axisymmetric collapse.

For further discussion of the first two decades of study of GW emission from stellar collapse see $[87,167,228]$. In the remainder of this section we will discuss more recent investigations.

\subsection{Bounce}

Most of the original GW studies of core-collapse supernovae focused on the bounce phase. Gravitational radiation will be emitted during the collapse/explosion of a core-collapse SN due to the star's changing quadrupole moment. A rough description of the possible evolution of the quadrupole moment is given in the remainder of this paragraph. During the first 100-250 ms of the collapse, as the core contracts and flattens, the magnitude of the quadrupole moment $\mathcal{I}_{j k}$ will increase. The contraction speeds up over the next $20 \mathrm{~ms}$ and the density distribution becomes more centrally condensed [201]. In this phase the core's shrinking size dominates its increasing deformation and the magnitude of $\mathcal{I}_{j k}$ decreases. As the core bounces, $\mathcal{I}_{j k}$ changes rapidly due to deceleration and rebound. If the bounce occurs because of nuclear pressure, its timescale will be $<1 \mathrm{~ms}$. If centrifugal forces play a role in halting the collapse, the bounce can last up to several ms [201]. The magnitude of $\mathcal{I}_{j k}$ will increase due to the core's expansion after bounce. As the resulting shock moves outward, the unshocked portion of the core will undergo oscillations, causing $\mathcal{I}_{j k}$ to oscillate as well. The shape of the core, the depth of the bounce, the bounce timescale, and the rotational 
energy of the core all strongly affect the GW emission. For further details see [86, 201, 167, 228].

The core collapse simulations of Mönchmeyer et al. [201] began with better iron core models and a more realistic microphysical treatment (including a realistic equation of state, electron capture, and a simple neutrino transport scheme) than any previous study of GW emission from axisymmetric stellar-core collapse. The shortcomings of their investigation included initial models that were not in rotational equilibrium, an equation of state that was somewhat stiff in the subnuclear regime, and the use of Newtonian gravity. Each of their four models had a different initial angular momentum profile. The rotational energies of the models ranged from $0.1-0.45$ of the maximum possible rotational energy.

The collapses of three of the four models of Mönchmeyer et al. were halted by centrifugal forces at subnuclear densities. This type of low $\rho_{\mathrm{c}}$ bounce had been predicted by Shapiro and Lightman [274] and Tohline [312] (in the context of the "fizzler" scenario for failed supernovae; see also [133, 134, 153]), and had been observed in earlier collapse simulations [210, 299]. Mönchmeyer and collaborators found that a bounce caused by centrifugal forces would last for several ms, whereas a bounce at nuclear densities would occur in $<1 \mathrm{~ms}$. They also determined that a subnuclear bounce produced larger amplitude oscillations in density and radius, with larger oscillation periods, than a bounce initiated by nuclear forces alone. They pointed out that these differences in timescale and oscillatory behavior should affect the GW signal. Therefore, the GW emission could indicate whether the bounce was a result of centrifugal or nuclear forces.

Mönchmeyer et al. [201] identified two different types of waveforms in their models (computed using the numerical quadrupole approximation discussed in Section 2.1). The waveforms they categorized as Type I (similar to those observed in previous collapse simulations [204, 89]) are distinguished by a large amplitude peak at bounce and subsequent damped ringdown oscillations. They noted that Type I signals were produced by cores that bounced at nuclear densities (or bounced at subnuclear densities if the cores had small ratios of radial kinetic to rotational kinetic energies). The quadrupole GW amplitude $A_{20}^{\mathrm{E} 2}$ for a Type I waveform is shown in Figure 10 (see [306, 350] for expressions relating $A_{20}^{\mathrm{E} 2}$ to $h$ ). The waveforms identified as Type II exhibit several maxima, which result from multiple bounces (see Figure 11 for an example of a Type II waveform). Note that the waveforms displayed in Figures 10 and 11 are from the study of Zwerger and Müller [350], discussed below.

The model of Mönchmeyer et al. [201] that bounced due to nuclear forces had the highest GW amplitude of all of their models, $h_{\mathrm{pk}} \sim 10^{-23}$ for a source distance $d=10 \mathrm{Mpc}$, and the largest emitted energy $E_{\mathrm{GW}} \sim 10^{47} \mathrm{erg}$. The accompanying power spectrum peaked in the frequency range $5 \times 10^{2}-10^{3} \mathrm{~Hz}$.

The most extensive Newtonian survey of the parameter space of axisymmetric, rotational core collapse is that of Zwerger and Müller [350]. They simulated the collapse of 78 initial models with varying amounts of rotational kinetic energy (reflected in the initial value of the stability parameter $\beta_{\mathrm{i}}$ ), differential rotation, and equation of state stiffness. In order to make this large survey tractable, they used a simplified equation of state and did not explicitly account for electron capture or neutrino transport. Their initial models were constructed in rotational equilibrium via the method of Eriguchi and Müller [79]. The models had a polytropic equation of state, with initial adiabatic index $\Gamma_{\mathrm{i}}=4 / 3$. Collapse was induced by reducing the adiabatic index to a value $\Gamma_{\mathrm{r}}$ in the range 1.28-1.325. The equation of state used during the collapse evolution had both polytropic and thermal contributions (note that simulations using more sophisticated equations of state get similar results [168]).

The major result of Zwerger and Müller's investigation was that the signal type of the emitted gravitational waveform in their runs was determined by the stiffness of the equation of state of the collapsing core (i.e., the value of $\Gamma_{\mathrm{r}}$ ). In their simulations, Type I signals (as labelled by Mönchmeyer et al. [201]) were produced by models with relatively soft equations of state, $\Gamma_{\mathrm{r}} \lesssim 1.31$. Type II signals were produced by the models with stiffer equations of state, $\Gamma_{\mathrm{r}} \gtrsim 1.32$. They found 


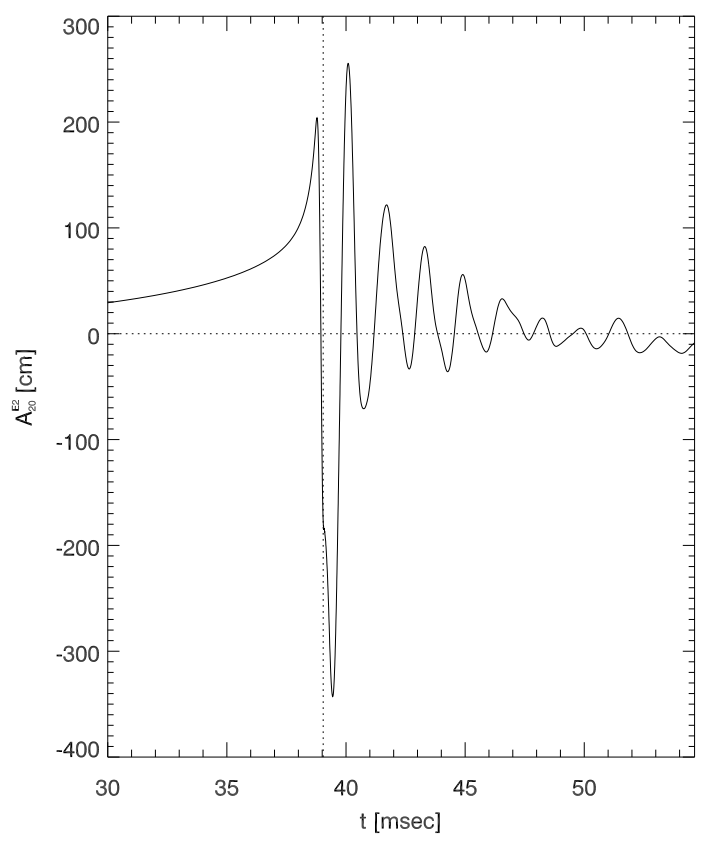

Figure 10: Type I waveform (quadrupole amplitude $A_{20}^{\mathrm{E} 2}$ as a function of time) from one of Zwerger and Müller's [350] simulations of a collapsing polytrope. The vertical dotted line marks the time at which the first bounce occurred. (Figure $5 \mathrm{~d}$ of [350]; used with permission.)

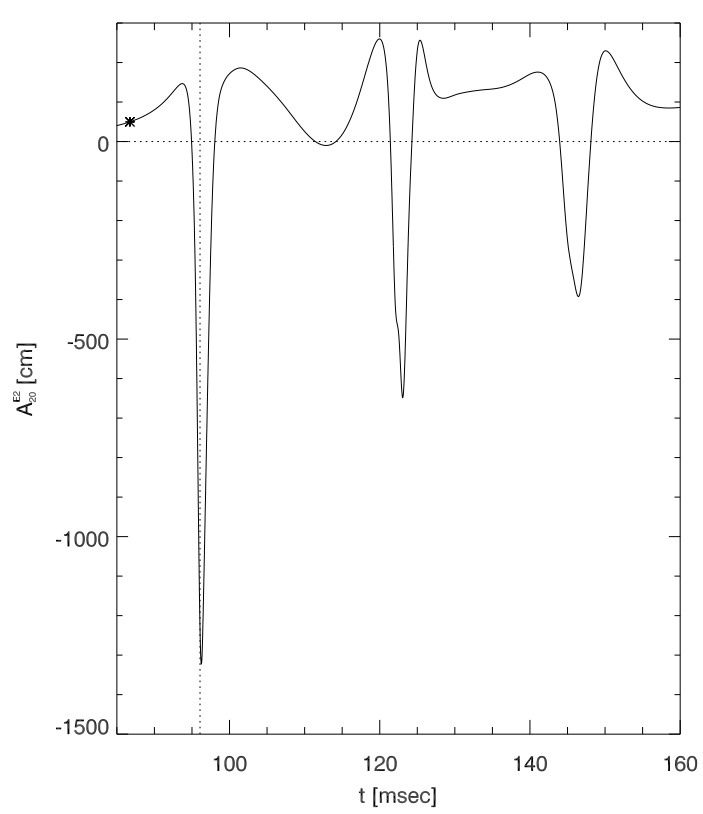

Figure 11: Type II waveform (quadrupole amplitude $A_{20}^{\mathrm{E} 2}$ as a function of time) from one of Zwerger and Müller's [350] simulations of a collapsing polytrope. The vertical dotted line marks the time at which bounce occurred. (Figure 5a of [350]; used with permission.) 


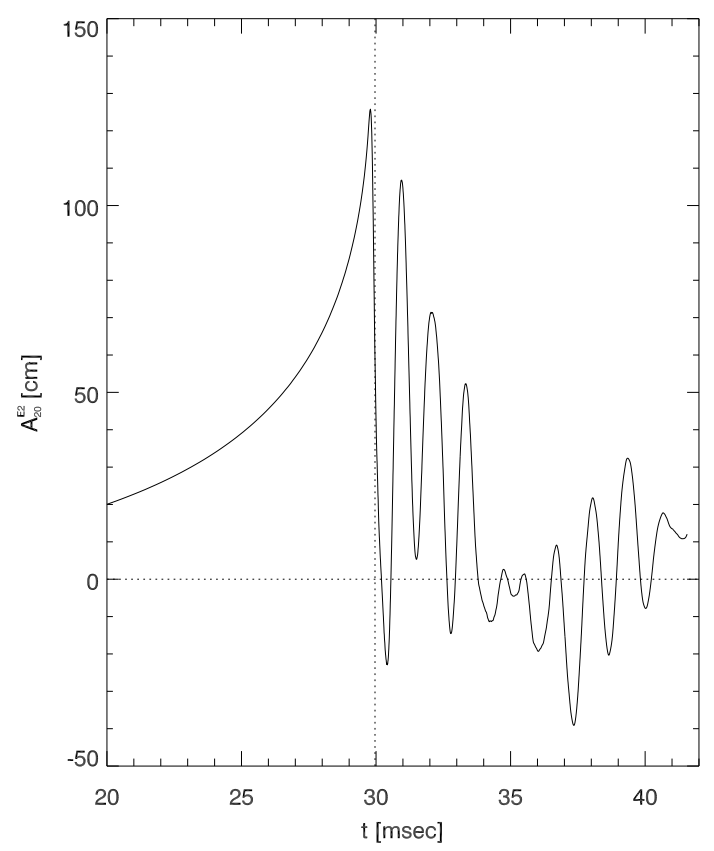

Figure 12: Type III waveform (quadrupole amplitude $A_{20}^{\mathrm{E} 2}$ as a function of time) from one of Zwerger and Müller's [350] simulations of a collapsing polytrope. The vertical dotted line marks the time at which bounce occurred. (Figure 5e of [350]; used with permission).

a smooth transition between these signal types if $\Gamma_{\mathrm{r}}$ was increased while all other parameters were held fixed. They also observed another class of signal, Type III, for their models with the lowest $\Gamma_{\mathrm{r}}(=1.28)$. Type III waveforms have a large positive peak just prior to bounce, a smaller negative peak just after bounce, and smaller subsequent oscillations with very short periods (see Figure 12). Type III signals were not observed in the evolution of strongly differentially-rotating $\Gamma_{\mathrm{r}}=1.28$ models and were also not seen in subsequent investigations $[168,230]$. Their waveforms were computed with the same technique as used in [201].

In contrast to the results of Mönchmeyer et al. [201], in Zwerger and Müller's investigation the value of $\rho_{\mathrm{c}}$ at bounce did not determine the signal type. Instead, the only effect on the waveform due to $\rho_{\mathrm{c}}$ was a decrease in $h_{\mathrm{pk}}$ in models that bounced at subnuclear densities. The effect of the initial value of $\beta_{\mathrm{i}}$ on $h_{\mathrm{pk}}$ was non-monotonic. For models with $\beta_{\mathrm{i}} \lesssim 0.1, h_{\mathrm{pk}}$ increased with increasing $\beta_{\mathrm{i}}$. This is because the deformation of the core is larger for faster rotators. However, for models with larger $\beta_{\mathrm{i}}, h_{\mathrm{pk}}$ decreases as $\beta_{\mathrm{i}}$ increases. These models bounce at subnuclear densities. Thus, the resulting acceleration at bounce and the GW amplitude are smaller. Zwerger and Müller found that the maximum value of $h_{\mathrm{pk}}$ for a given sequence was reached when $\rho_{\mathrm{c}}$ at bounce was just less than $\rho_{\text {nuc }}$. The degree of differential rotation did not have a large effect on the emitted waveforms computed by Zwerger and Müller. However, they did find that models with soft equations of state emitted stronger signals as the degree of differential rotation increased.

The models of Zwerger and Müller that produced the largest GW signals fell into two categories: those with stiff equations of state and $\beta_{\mathrm{i}}<0.01$; and those with soft equations of state, $\beta_{\mathrm{i}} \geq 0.018$, and large degrees of differential rotation. The GW amplitudes emitted during their simulations were in the range $4 \times 10^{-25} \lesssim h \lesssim 4 \times 10^{-23}$, for $d=10 \mathrm{Mpc}$. The corresponding energies ranged from $10^{44} \lesssim E_{\mathrm{GW}} \lesssim 10^{47}$ erg. The peaks of their power spectra were between $500 \mathrm{~Hz}$ and $1 \mathrm{kHz}$. Such signals would fall just outside of the range of LIGO-II.

Yamada and Sato [337] used techniques very similar to those of Zwerger and Müller [350] in 
their core collapse study. Their investigation revealed that the $h_{\mathrm{pk}}$ for Type I signals became saturated when the dimensionless angular momentum of the collapsing core, $q=J /(2 G M / c)$, reached $\sim 0.5$. They also found that $h_{\mathrm{pk}}$ was sensitive to the stiffness of the equation of state for densities just below $\rho_{\text {nuc }}$. The characteristics of the GW emission from their models were similar to those of Zwerger and Müller. A series of new results (e.g., [168, 169, 232, 233, 68, 69]) have essentially confirmed these results.

Kotake et al. [169] found that magnetic fields can lower these amplitudes (they lower the rotation rate) by $\sim 10 \%$, but realistic stellar profiles can lower the amplitudes by a factor of $\gtrsim 4-10[230$, 209], restricting the detectability of supernovae to within our galaxy $(\lesssim 10 \mathrm{kpc})$. Obviously, this effect depends on the strength of the magnetic fields and Obergaulinger et al. [227, 226] studied this effect by using a variety of magnetic-field strengths. General-relativistic magneto-hydrodynamic calculations of the bounce phase have confirmed these results [48, 47]. Magnetohydrodynamic calculations are becoming more common, gradually increasing our intuition about this important piece of physics [267, 266, 300].

Fryer and Warren [112] performed the first 3D collapse simulations to follow the entire collapse through explosion. They used a smoothed particle hydrodynamics code, a realistic equation of state, the flux-limited gray diffusion approximation for neutrino transport, and Newtonian spherical gravity. The gray approximation is a limiting assumption and a multi-group calculation can produce different results [34, 325]. Their initial model was non-rotating. Thus, no bar-mode instabilities could develop during their simulations. The only GW-emitting mechanism present in their models was convection in the core. The maximum amplitude $h$ of this emission, computed in the quadrupole approximation, was $\sim 3 \times 10^{-26}$, for $d=10 \mathrm{Mpc}$ [108]. In later work, Fryer \& Warren [113] included full Newtonian gravity through a tree algorithm and studied the rotating progenitors from Fryer \& Heger [103]. By the launch of the explosion, no bar instabilities had developed. This was because of several effects: they used slowly rotating, but presumably realistic, progenitors [103], the explosion occured quickly for their models ( $\lesssim 100 \mathrm{~ms})$ and, finally, because much of the high angular momentum material did not make it into the inner core. These models have been further studied for the GW signals [107]. The fastest-rotating models achieved a signal of $h \sim 2 \times 10^{-24}$ for $d=10 \mathrm{Mpc}$ and characteristic frequencies of $f_{\mathrm{GW}} \sim 1000 \mathrm{~Hz}$. For supernovae occuring within the galaxy, such a signal is detectable by LIGO-II. Dimmelmeier et al. [68, 69] and Ott et al. [232, 233] have studied these rotating progenitors, producing amplitudes on par with these results, but providing much more detailed information, including spectra. These models included full general relativity and are discussed in Section 4.1.1.

Fryer and collaborators have also modeled asymmetric collapse and asymmetric explosion calculations in three dimensions [98, 110]. These calculations will be discussed in Sections 4.2 and 4.4 .

\subsubsection{General relativistic calculations}

General-relativistic effects oppose the stabilizing influence of rotation in pre-collapse cores. Thus, stars that might be prevented from collapsing due to rotational support in the Newtonian limit may collapse when general-relativistic effects are considered. Furthermore, general relativity will cause rotating stars undergoing collapse to bounce at higher densities than in the Newtonian case [302, 350, 245, 33].

The full collapse simulations of Fryer and Heger [103] followed the axisymmetric evolution of the collapse, bounce and explosion including equation of state and neutrino transport from which the resultant GW emission has been studied [106, 108]. Fryer and Heger only include the effects of general relativity in the monopole approximation. This ignores many feedback mechanisms. The GW emission from these simulations was evaluated with either the quadrupole approximation or simpler estimates (see below). 
The work of Fryer and Heger [103] was an improvement over past collapse investigations because it starts with rotating progenitors evolved to collapse with a stellar evolution code (which incorporates angular momentum transport via an approximate diffusion scheme) [135], incorporates realistic equations of state and neutrino transport, and follows the collapse to late times. The values of total angular momentum of the inner cores of Fryer and Heger $\left(0.95-1.9 \times 10^{49} \mathrm{~g} \mathrm{~cm}^{2} \mathrm{~s}^{-1}\right)$ are lower than has often been assumed in studies of the GW emission from core collapse. Note that the total specific angular momentum of these core models may be lower by about a factor of 10 if magnetic fields were included in the evolution of the progenitors [2, 290, 138].

FHH's [106] numerical quadrupole estimate of the GWs from oscillations observed in the collapse simulations of Fryer and Heger [103] predicts an upper limit of the peak dimensionless amplitude $h_{\mathrm{pk}}=4.1 \times 10^{-23}$ (for $d=10 \mathrm{Mpc}$ ), emitted at $f_{\mathrm{GW}} \approx 20 \mathrm{~Hz}$. The radiated energy $E_{\mathrm{GW}} \sim 2 \times 10^{44} \mathrm{erg}$. This signal would be just out of the detectability range of the LIGO-II detector. However, most simulations predict lower amplitudes peaking at different frequencies.

Dimmelmeier and collaborators $[68,69]$ have now completed a very extensive study of axisymmetric collapse including modern equations of state and a recipe for the evolution of the electron fraction. The range of their results was consistent with the upper limits placed by FHH, with their strongest signal have a peak dimensionless amplitude $h_{\mathrm{pk}}=1.0 \times 10^{-23}$ (for $d=10 \mathrm{Mpc}$ ).

General relativity has been more fully accounted for in the core collapse studies of Dimmelmeier, Font, and Müller [65, 66, 67] and Shibata and collaborators [281, 283], which build on the Newtonian, axisymmetric collapse simulations of Zwerger and Müller [350]. In all, they have followed the collapse evolution of 26 different models, with both Newtonian and general-relativistic simulations. As in the work of Zwerger and Müller, the different models are characterized by varying degrees of differential rotation, initial rotation rates, and adiabatic indices. They use the conformally-flat metric to approximate the spacetime geometry [57] in their relativistic hydrodynamics simulations. This approximation gives the exact solution to Einstein's equations in the case of spherical symmetry. Thus, as long as the collapse is not significantly aspherical, the approximation is relatively accurate. However, the conformally-flat condition does eliminate GW emission from the spacetime. Because of this, Dimmelmeier, Font, and Müller used the quadrupole approximation to compute the characteristics of the emitted GW signal (see [350] for details).

The general-relativistic simulations of Dimmelmeier et al. showed the three different types of collapse evolution (and corresponding gravitational radiation signal) seen in the Newtonian simulations of Zwerger and Müller (regular collapse - Type I signal; multiple bounce collapse Type II signal; and rapid collapse - Type III signal). Obergaulinger et al. [227, 226] identified a fourth type caused by the effects of magnetic fields. However, relativistic effects sometimes led to a different collapse type than in the Newtonian case. This is because general relativity did indeed counteract the stabilizing effects of rotation and led to much higher bounce densities (up to $700 \%$ higher $)^{11}$. They found that multiple bounce collapse is much rarer in general-relativistic simulations (occurring in only two of their models). When multiple bounces do occur, relativistic effects shorten the time interval between bounces by up to a factor of four. Movies of the simulations of four models from Dimmelmeier et al. [67] are shown in Figures 13, 14, 15, and 16. The four evolutions shown include a regular collapse (Movie 13), a rapid collapse (Movie 14), a multiple bounce collapse (Movie 15), and a very rapidly- and differentially-rotating collapse (Movie 16). The left frames of each movie contain the $2 \mathrm{D}$ evolution of the logarithmic density. The upper and lower right frames display the evolutions of the GW amplitude and the maximum density, respectively. These movies can also be viewed at [124].

Dimmelmeier et al. found that models for which the collapse type was the same in both Newtonian and relativistic simulations had lower GW amplitudes $h_{\mathrm{pk}}$ in the relativistic case. This is because the Newtonian models were less compact at bounce and thus had material with higher densities and velocities at larger radii. Both higher and lower values of $h_{\mathrm{pk}}$ were observed in mod-

11 This "softening" of the equation of state has been studied for three decades [322]. 

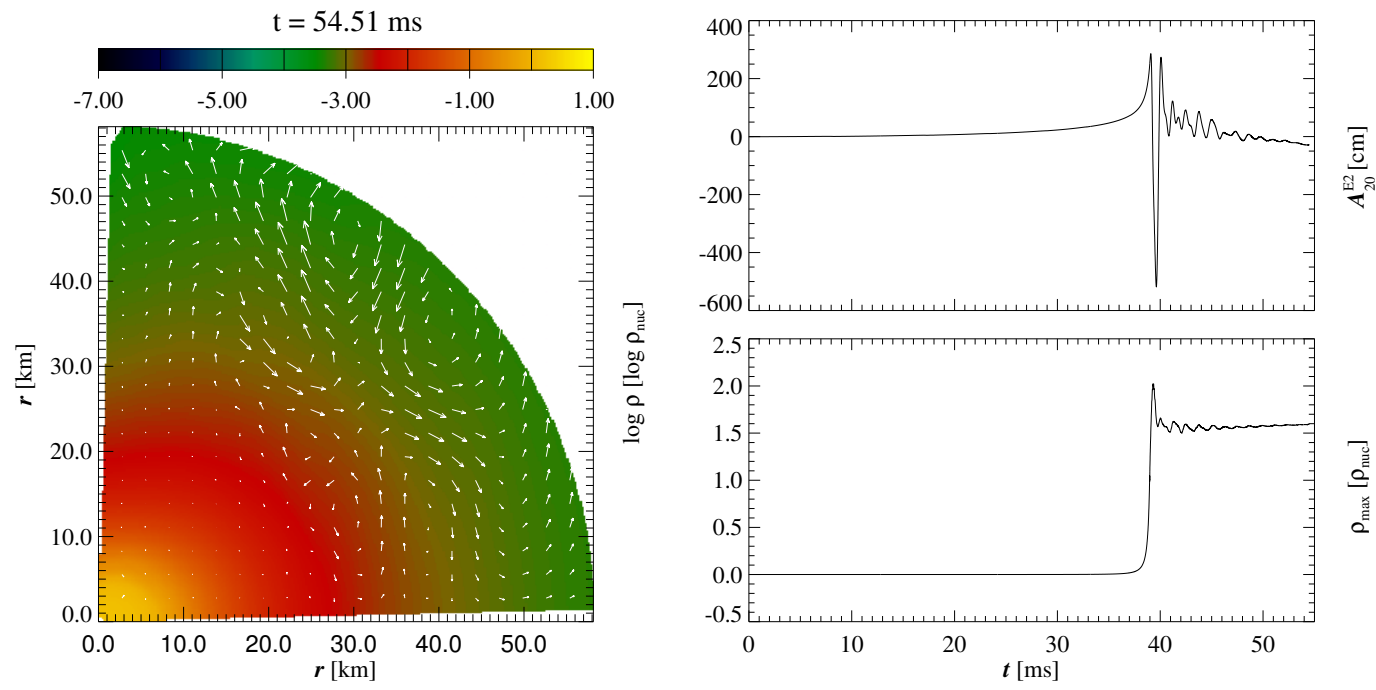

Figure 13: Still from a movie showing The evolution of the regular collapse model A3B2G4 of Dimmelmeier et al. [67]. The left frame contains the 2D evolution of the logarithmic density. The upper and lower right frames display the evolutions of the gravitational wave amplitude and the maximum density, respectively. (To watch the movie, please go to the online version of this review article at http://www.livingreviews.org/lrr-2011-1.)
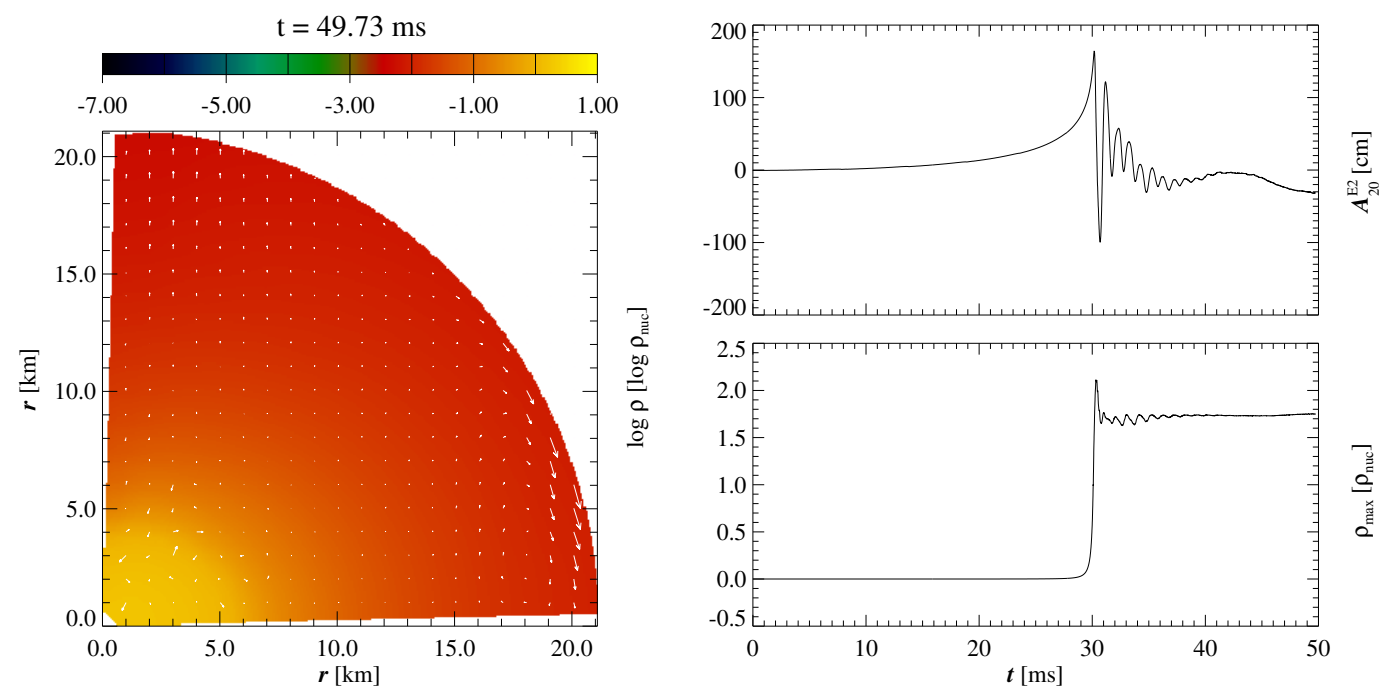

Figure 14: Still from a movie showing Same as Movie 13, but for rapid collapse model A3B2G5 of Dimmelmeier et al. [67]. (To watch the movie, please go to the online version of this review article at http://www. livingreviews .org/lrr-2011-1.) 

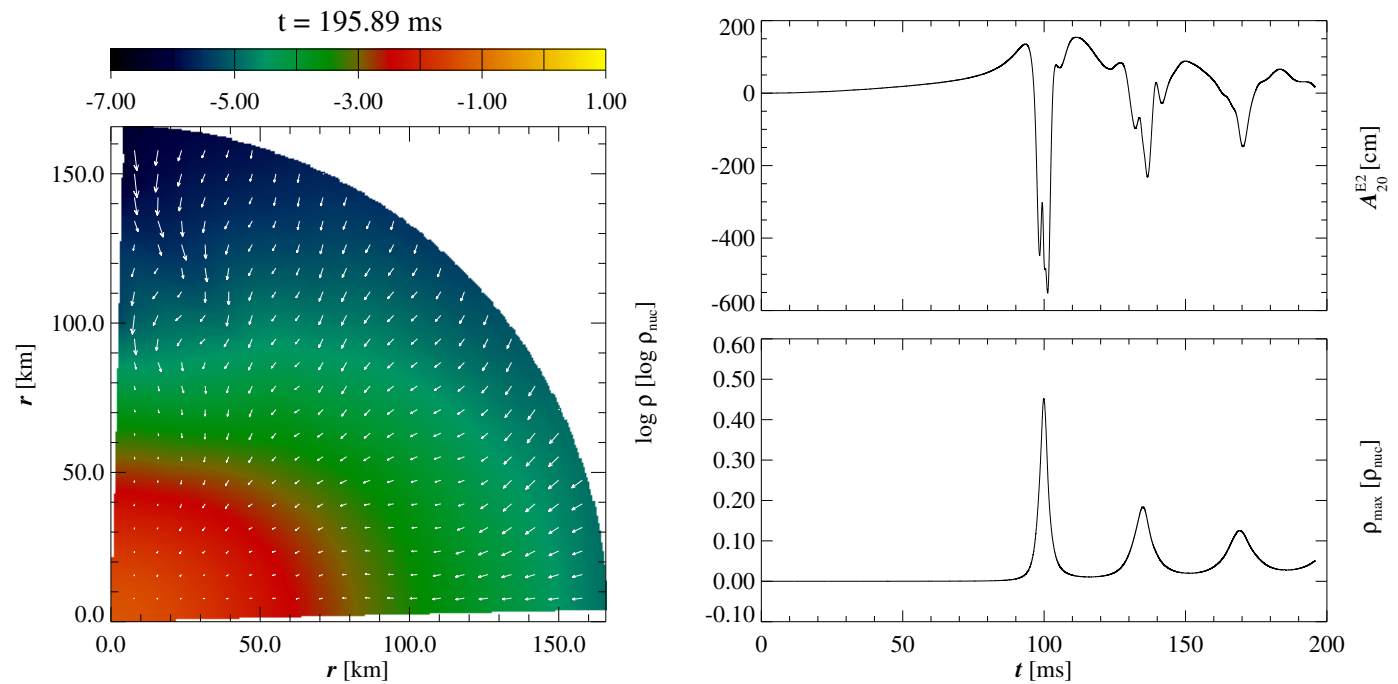

Figure 15: Still from a movie showing Same as Movie 13, but for multiple collapse model A2B4G1 of Dimmelmeier et al. [67]. (To watch the movie, please go to the online version of this review article at http://www. livingreviews . org/lrr-2011-1.)
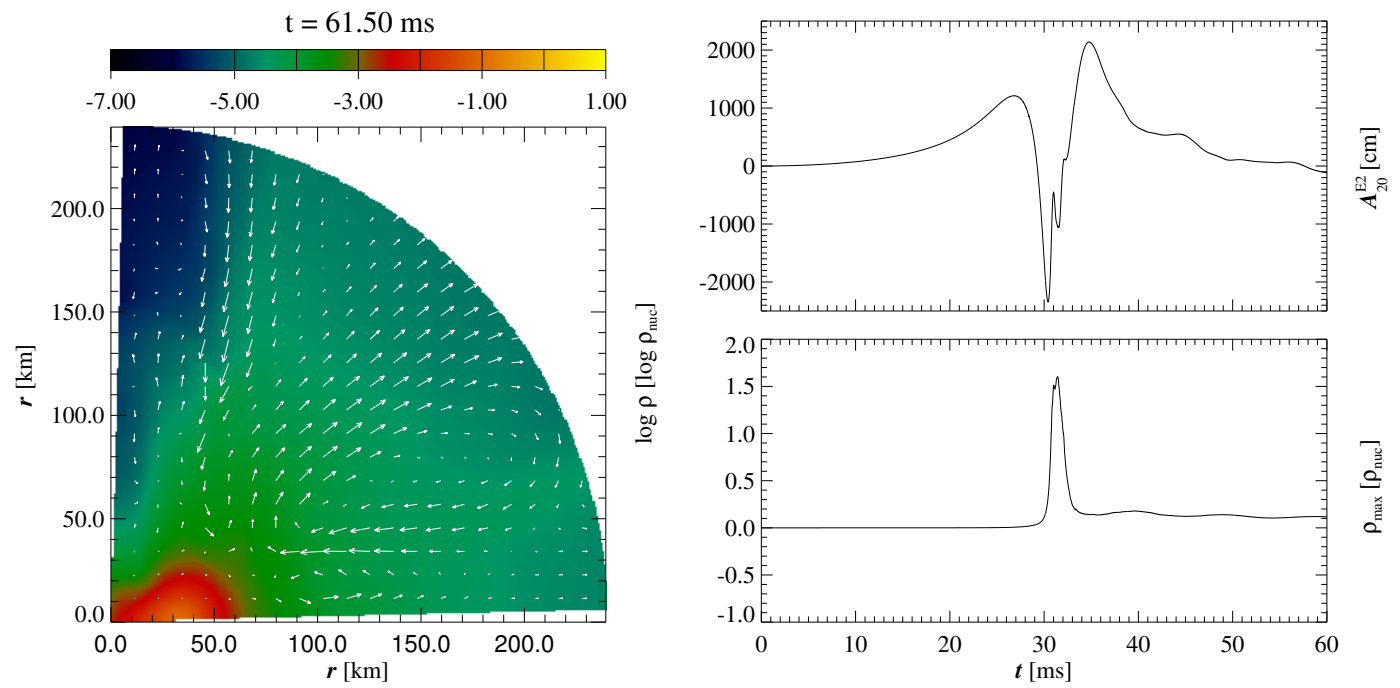

Figure 16: Still from a movie showing Same as Movie 13, but for rapid, differentially-rotating collapse model A4B5G5 of Dimmelmeier et al. [67]. (To watch the movie, please go to the online version of this review article at http://www.livingreviews.org/lrr-2011-1.) 
els for which the collapse type changed. Overall, the range of $h_{\mathrm{pk}}\left(4 \times 10^{-24}-3 \times 10^{-23}\right.$, for a source located at $10 \mathrm{Mpc}$ ) seen in the relativistic simulations was quite close to the corresponding Newtonian range. The average $E_{\mathrm{GW}}$ was somewhat higher in the relativistic case $\left(1.5 \times 10^{47} \mathrm{erg}\right.$ compared to the Newtonian value of $6.4 \times 10^{46} \mathrm{erg}$ ). The overall range of GW frequencies observed in their relativistic simulations $(60-1000 \mathrm{~Hz})$ was close to the Newtonian range. They did note that relativistic effects always caused the characteristic frequency of emission, $f_{\mathrm{GW}}$, to increase (up to five-fold). Studies of non-linear pulsations in neutron stars expect high frequencies between $1.8-3.6 \mathrm{kHz}$ [293]. For most of their models, this increase in $f_{\mathrm{GW}}$ was not accompanied by an increase in $h_{\mathrm{pk}}$. This means that relativistic effects could decrease the detectability of GW signals from some core collapses. However, the GW emission from the models of Dimmelmeier et al. could be detected by the first generation of ground-based interferometric detectors if the sources were fortuitously located in the Local Group of galaxies. A catalog containing the signals and spectra of the GW emission from all of their models can be found at [292].

Ott et al. [232, 233] argue that the GW signal from the collapse, bounce, and early postbounce phases of the core collapse evolution is much more generic than many of these past results show, arguing that variations in the rotation (within the limits studied in their calculations) do not alter the signal significantly. If these results are confirmed, firm estimates can be provided to the GW detector community. 


\subsection{Convection}

As we discussed at the beginning of Section 3, it is becoming increasingly accepted that convection above the proto neutron star plays a major role in the supernova explosion mechanism (e.g., Figure 1). Convectively-driven inhomogeneities in the density distribution of the outer regions of the nascent neutron star and anisotropic neutrino emission are other sources of GW emission during the collapse/explosion [37, 206]. Inside the proto neutron star, GW emission from these processes results from small-scale asphericities, unlike the large-scale motions responsible for GW emission from aspherical collapse and non-axisymmetric global instabilities. But the SASI and low-mode convection do produce large-scale accelerated mass motions [165, 213]. Note that Rayleigh-Taylor instabilities in the exploding star also induce time-dependent quadrupole moments at composition interfaces in the stellar envelope. However, the resultant GW emission is too weak to be detected because the Rayleigh-Taylor instabilities occur at very large radii (with low velocities) [206].

\subsubsection{Asymmetric collapse}

Since convection was suggested as a key ingredient in the explosion, it has been postulated that asymmetries in the convection can produce the large proper motions observed in the pulsar population [139]. Convection asymmetries can either be produced by asymmetries in the progenitor star that grow during collapse or by instabilities in the convection itself. Burrows and Hayes [37] proposed that asymmetries in the collapse could produce pulsar velocities. The idea behind this work was that asymmetries present in the star prior to collapse (in part due to convection during silicon and oxygen burning) will be amplified during the collapse [13, 172]. These asymmetries will then drive asymmetries in the convection and ultimately, the supernova explosion. Burrows and Hayes [37] found that not only could they produce strong motions in the nascent neutron star, but detectable GW signals. The peak amplitude calculated was $h_{\mathrm{pk}} \sim 3 \times 10^{-24}$, for a source located at $10 \mathrm{Mpc}$.

Fryer [98] was unable to produce the large neutron star velocities seen by Burrows and Hayes [37] even after significantly increasing the level of asymmetry in the initial star in excess of $25 \%$. More recent results by Burrows' group [212] suggest that the Burrows and Hayes results were not quantitatively correct. However, the GW signal produced by the more recent and the original simulations is comparable. Figure 17 shows the gravitational waveform from the Burrows \& Hayes simulation (including separate matter and neutrino contributions). Note that the neutrinos dominate the amplitude of the GW signal. As we shall see in Section 4.4, the neutrino contribution may dominate the GW signal from many asymmetric collapse simulations. Figure 18 shows the matter contribution to the gravitational waveforms for the Fryer results.

The study by Nazin and Postnov [220] predicts a lower limit for $E_{\mathrm{GW}}$ emitted during an asymmetric core-collapse SN (where such asymmetries could be induced by both aspherical mass motion and neutrino emission). They assume that observed pulsar kicks are solely due to asymmetric collapse. They suggest that the energy associated with the kick $\left(M v^{2} / 2\right.$, where $M$ and $v$ are the mass and velocity of the neutron star) can be set as a lower limit for $E_{\mathrm{GW}}$ (which can be computed without having to know the mechanism behind the asymmetric collapse). From observed pulsar proper motions, they estimate the degree of asymmetry $\epsilon$ present in the collapse and the corresponding characteristic GW amplitude $(h \propto \sqrt{\epsilon})$. This amplitude is $3 \times 10^{-25}$ for a source located at $10 \mathrm{Mpc}$ and emitting at $f_{\mathrm{GW}}=1 \mathrm{kHz}$.

\subsubsection{Proto neutron star convection}

Müller and Janka [208] performed both 2D and 3D simulations of convective instabilities in the proto neutron star and hot bubble regions during the first second of the explosion phase of a Type II SN. They numerically computed the GW emission from the convection-induced aspherical 


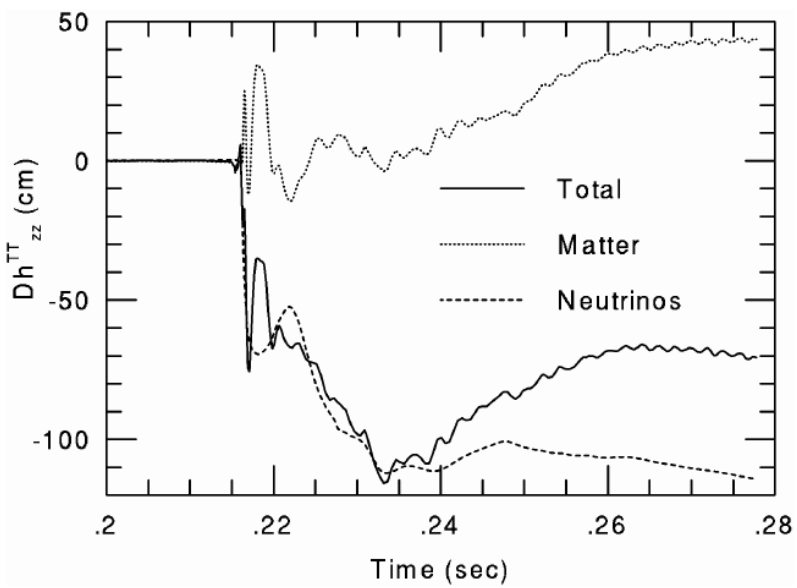

Figure 17: The gravitational waveform (including separate matter and neutrino contributions) from the collapse simulations of Burrows and Hayes [37]. The curves plot the GW amplitude of the source as a function of time. (Figure 3 of [37]; used with permission.)
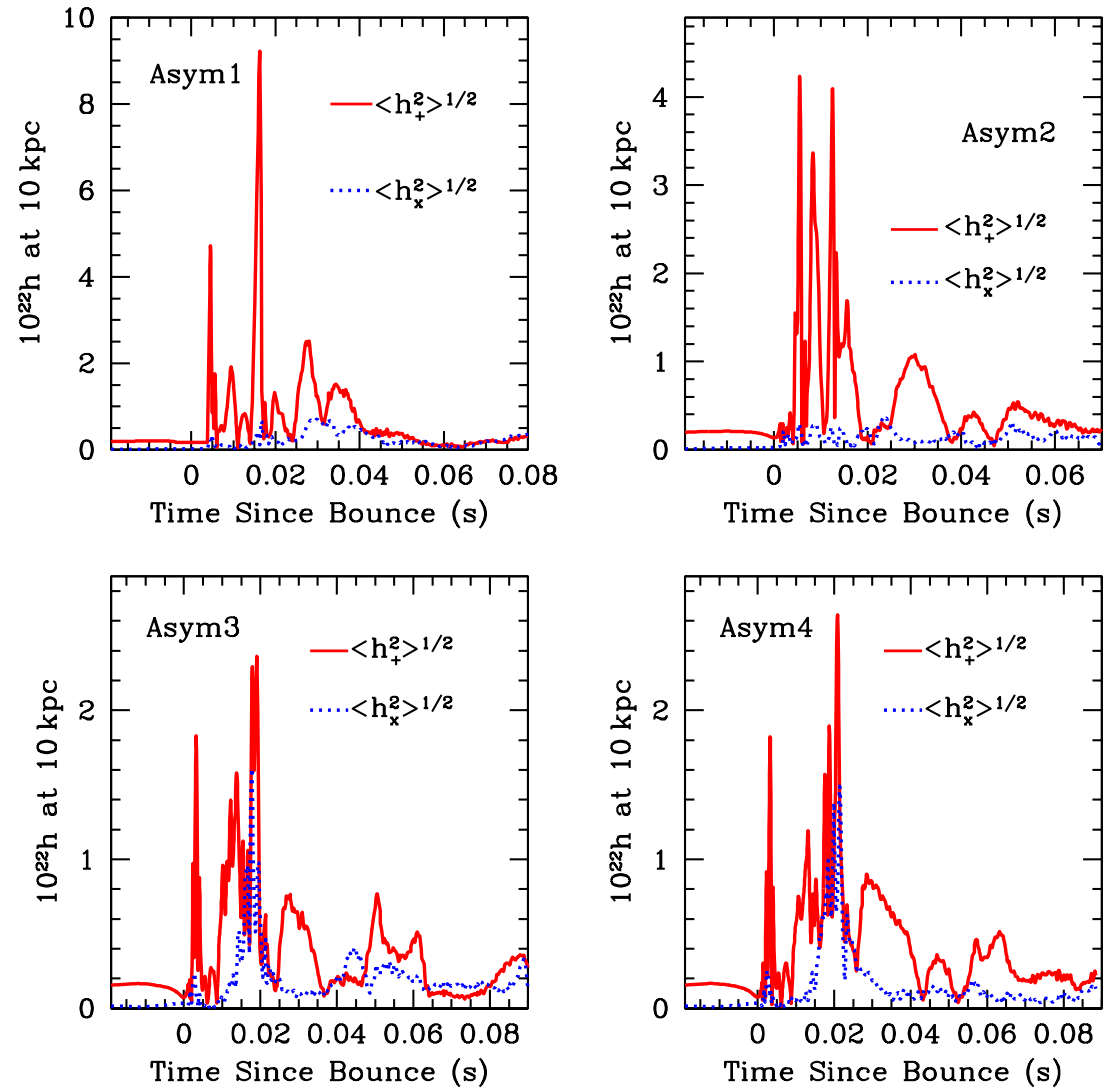

Figure 18: The gravitational waveform for matter contributions from the asymmetric collapse simulations of Fryer et al. [107]. The curves plot the GW amplitude of the source as a function of time. (Figure 3 of [107]; used with permission.) 
mass motion and neutrino emission in the quadrupole approximation (for details, see Section 3 of their paper [208]).

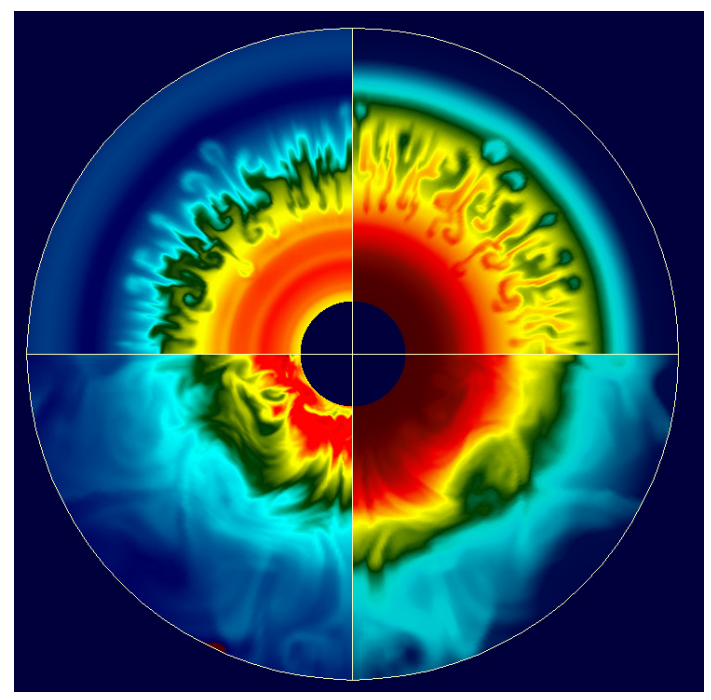

Figure 19: Convective instabilities inside the proto neutron star in the $2 \mathrm{D}$ simulation of Müller and Janka [208]. The evolutions of the temperature (left panels) and logarithmic density (right panels) distributions are shown for the radial region $15-95 \mathrm{~km}$. The upper and lower panels correspond to times 12 and $21 \mathrm{~ms}$, respectively, after the start of the simulation. The temperature values range from $2.5 \times 10^{10}$ to $1.8 \times 10^{11} \mathrm{~K}$. The values of the logarithm of the density range from 10.5 to $13.3 \mathrm{~g} \mathrm{~cm}^{-3}$. The temperature and density both increase as the colors change from blue to green, yellow, and red. (Figure 7 of [208]; used with permission.)

For typical iron core masses, the convectively unstable region in the proto neutron star extends over the inner $0.7-1.20 M_{\odot}$ of the core mass (this corresponds to a radial range of $\sim 10-50 \mathrm{~km}$ ). The convection in this region, which begins approximately $10-20 \mathrm{~ms}$ after the shock forms and may last for $\sim 20 \mathrm{~ms}-1 \mathrm{~s}$, is caused by unstable gradients in entropy and/or lepton number resulting from the stalling of the prompt shock and deleptonization outside the neutrino sphere. Müller and Janka's simulations of convection in this region began with the 1D, non-rotating, $12 \mathrm{~ms}$ post-bounce model of Hillebrandt [142]. This model included general-relativistic corrections that had to be relaxed away prior to the start of the Newtonian simulations. Neutrino transport was neglected in these runs (see Section 2.1 of [208] for justification); however, a sophisticated equation of state was utilized. Figure 19 shows the evolution of the temperature and density distributions in the 2D simulation of Müller and Janka.

The peak GW amplitude resulting from convective mass motions in these simulations of the proto neutron star was $\sim 3 \times 10^{-24}$ in $2 \mathrm{D}$ and $\sim 2 \times 10^{-25}$ in 3D, for $d=10 \mathrm{Mpc}$. More recent calculations get amplitudes of $\sim 10^{-26}$ in $2 \mathrm{D}$ [209] and $\sim 3-5 \times 10^{-26}$ in 3D [107]. The emitted energy was $9.8 \times 10^{44} \mathrm{erg}$ in $2 \mathrm{D}$ and $1.3 \times 10^{42} \mathrm{erg}$ in $3 \mathrm{D}$. The power spectrum peaked at frequencies of $200-600 \mathrm{~Hz}$ in $2 \mathrm{D}$ and $100-200 \mathrm{~Hz}$ in 3D. Such signals would not be detectable with LIGO-II. The reasons for the differences between the $2 \mathrm{D}$ and $3 \mathrm{D}$ results include smaller convective elements and less under and overshooting in 3D. The relatively low angular resolution of the 3D simulations may also have played a role. The quadrupole GW amplitude $A_{20}^{\mathrm{E} 2}$ from the $2 \mathrm{D}$ simulation is shown in the upper left panel of Figure 20 (see $[350,306]$ for expressions relating $A_{20}^{\mathrm{E} 2}$ to $h$ ). 

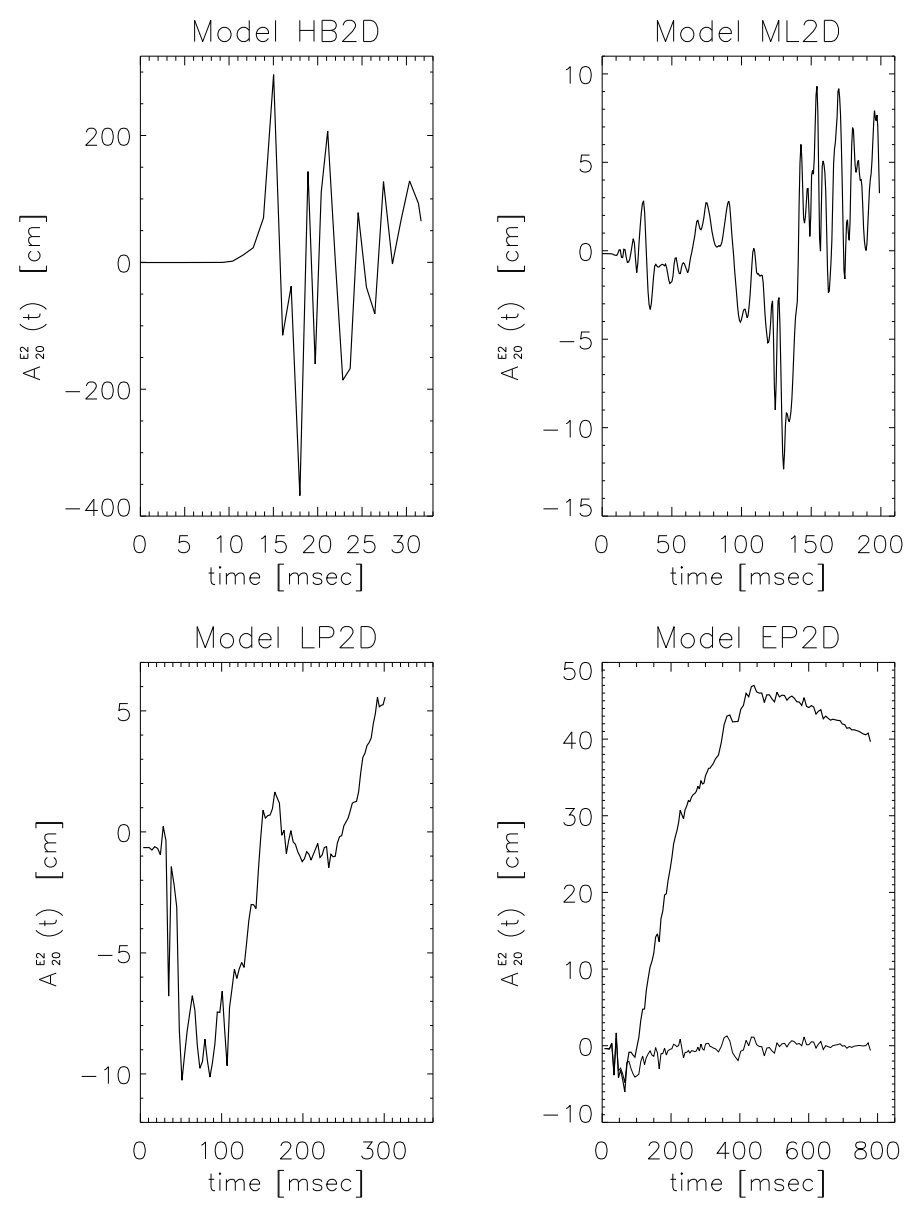

Figure 20: Quadrupole amplitudes $A_{20}^{\mathrm{E} 2}[\mathrm{~cm}]$ from convective instabilities in various models of [208]. The upper left panel is the amplitude from a $2 \mathrm{D}$ simulation of proto neutron star convection. The other three panels are amplitudes from $2 \mathrm{D}$ simulations of hot bubble convection. The imposed neutrino flux in the hot bubble simulations increases from the top right model through the bottom right model. (Figure 18 of [208]; used with permission.) 


\subsubsection{Convection above the proto neutron star}

Convection in the hot bubble region between the shock and neutrino sphere arises because of an unstable entropy gradient resulting from the outward moving shock and subsequent neutrino heating. Figure 21 shows a movie of the development of this entropy-driven convection. This unstable region extends over the inner mass range 1.25-1.40 $M_{\odot}$ (corresponding to a radial range of $\sim 100-1000 \mathrm{~km}$ ). Convection in the hot bubble begins $\sim 50-80 \mathrm{~ms}$ after shock formation and lasts for $\sim 100-500 \mathrm{~ms}$. Only 2D simulations were performed in this case. These runs started with a 25 ms post-bounce model provided by Bruenn [32]. A simple neutrino transport scheme was used in the runs and an imposed neutrino flux was located inside the neutrino sphere. Due to computational constraints, the computational domain did not include the entire convectively unstable region inside the proto neutron star (thus, this set of simulations only accurately models the convection in the hot bubble region, not in the proto neutron star).

The peak GW amplitude resulting from these $2 \mathrm{D}$ simulations of convective mass motions in the hot bubble region was $h_{\mathrm{pk}} \approx 5 \times 10^{-25}$, for $d=10 \mathrm{Mpc}$. The emitted energy was $\lesssim 2 \times 10^{42} \mathrm{erg}$. The energy spectrum peaked at frequencies of $50-200 \mathrm{~Hz}$. As the explosion energy was increased (by increasing the imposed neutrino flux), the violent convective motions turn into simple rapid expansion. The resultant frequencies drop to $f_{\mathrm{GW}} \sim 10 \mathrm{~Hz}$. The amplitude of such a signal would be too low to be detectable with LIGO-II.

The case for GWs from convection-induced asymmetric neutrino emission has also varied with time. Müller and Janka estimated the GW emission from the convection-induced anisotropic neutrino radiation in their simulations (see [208] for details). They found that the amplitude of the GWs emitted can be a factor of 5-10 times higher than the GW amplitudes resulting from convective mass motion. More recent simulations by Müller et al. [209] argue that the GWs produced by asymmetric neutrino emission is weaker than that of the convective motions. But Marek et al. [196] have argued strongly that calculating the GW signal from asymmetries in neutrinos is extremely difficult and detailed neutrino transport is required to determine the GW signal from neutrinos.

\subsubsection{Low-mode convection and the standing accretion-shock instability}

Our understanding of the convective engine is deepening with time. The latest focus of attention has been the SASI instability, which produces, at late times, extremely low mode convection. This topic is currently a matter of heated debate. Whether this instability dominates at late times, or whether the late-time, low-mode convection is simply the merger of convective cells [139] is, in the opinion of these authors, yet to be conclusively determined. Convection is very difficult to simulate and has been studied for many decades on a variety of applications from the combustion engine to astrophysics, with no accepted resolution. But many groups are now finding low-mode convection above the proto neutron star (which can heighten the GW emission) and all agree that this occurs at late times (more than a few hundred milliseconds after bounce). As we have shown in Figure 5, such late explosions will be weak and, if the assumptions of that analysis are correct, these late explosion mechanisms cannot explain the observed core-collapse supernovae. Marek \& Janka [195] believe otherwise. Certainly, if material can continue to accrete onto the neutron star after the launch of the explosion, which it does in some of the recent results of the Mezzacappa team (in preparation), stronger explosions may be produced.

In addition, Burrows et al. [40] found that the downstreams in the SASI can drive oscillations in the neutron star, which may also be a source for GWs (see Figure 22). Many groups have shown that the convection does not, and can not in semi-analytic studies, excite the strong oscillations observed [116, 343, 195], but see Weinberg \& Quataert [327]. When low-mode convection does occur as in the SASI, a GW signal is produced and has been modeled by several groups [85, $229,228,196,165]$. Most find that the actual matter signal is quite weak: $h_{\mathrm{pk}} \approx 5 \times 10^{-25}$, for 

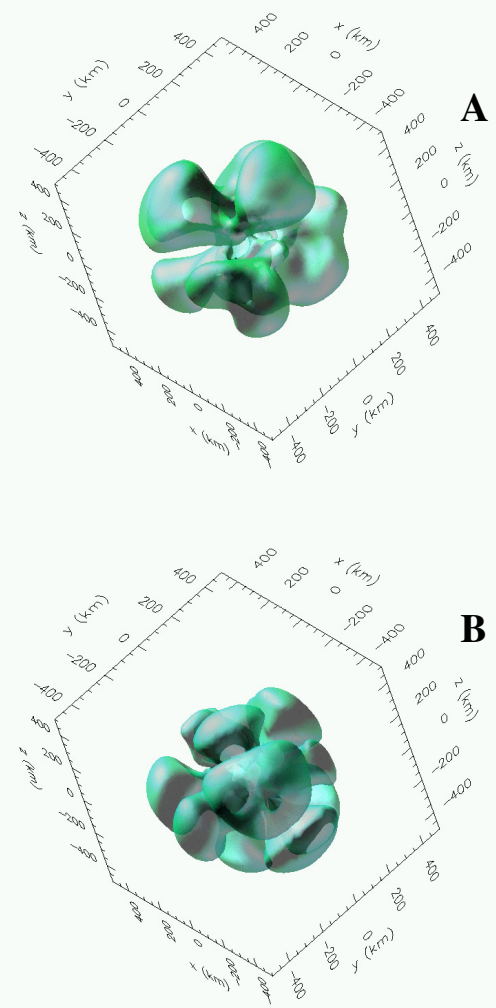

B

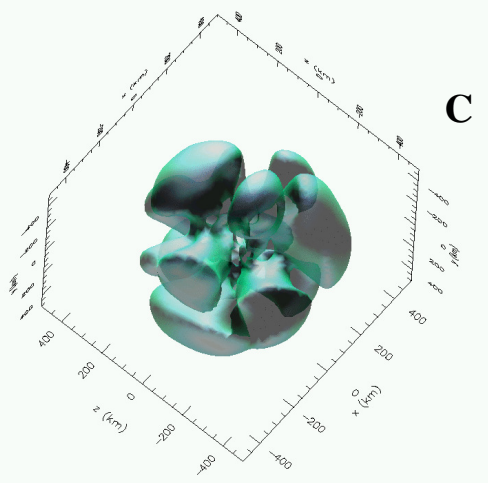

Figure 21: Still from a movie showing Isosurface of material with radial velocities of $1000 \mathrm{~km} \mathrm{~s}^{-1}$ for three different simulation resolutions. The isosurface outlines the outward moving convective bubbles. The open spaces mark the downflows. Note that the upwelling bubbles are large and have very similar size scales to the two-dimensional simulations. From Fryer \& Warren [112]. (To watch the movie, please go to the online version of this review article at http://www.livingreviews.org/lrr-2011-1.) 
$d=10 \mathrm{Mpc}$, but the SASI can drive oscillations in the neutron star that produce a potentially important neutrino signal.

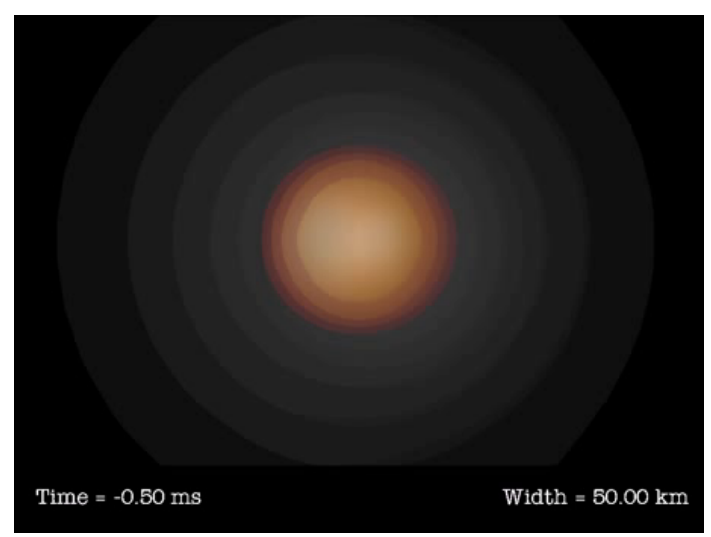

Figure 22: Still from a movie showing The oscillation of the proto neutron star caused by downstreams in the SASI-induced convective region above the proto neutron star. From Burrows et al. [40]. (To watch the movie, please go to the online version of this review article at http://www.livingreviews.org/ $\operatorname{lrr}-2011-1$. 


\subsection{Bar modes}

Rotational instabilities in proto neutron stars, if they exist, could be very powerful GW sources. Global rotational instabilities in a collapsed core lead to rapid variations in the quadrupole moment, and hence, strong GW emission. Global rotational instabilities in fluids arise from nonaxisymmetric modes $e^{ \pm i m \phi}$, where $m=2$ is known as the "bar mode" [302, 5]. It is convenient to parameterize a system's susceptibility to these modes by the stability parameter $\beta=T_{\text {rot }} /|W|$. Here, $T_{\text {rot }}$ is the rotational kinetic energy and $W$ is the gravitational potential energy. Dynamical rotational instabilities, driven by Newtonian hydrodynamics and gravity, develop on the order of the rotation period of the object. For the uniform-density, incompressible, uniformly-rotating MacLaurin spheroids, the dynamical bar-mode instability sets in at $\beta_{\mathrm{d}} \approx 0.27$. These instabilities have been confirmed by a number of studies $[276,192,74,313,331,314,147,289,148,146,237$, $315,154,221,30,184,232,233]$, and we will discuss some of these results here. At lower rotation rates, a secular instability may develop that produces bar modes if viscous or gravitational radiation reaction forces can redistribute the angular momentum [52, 173, 171, 236].

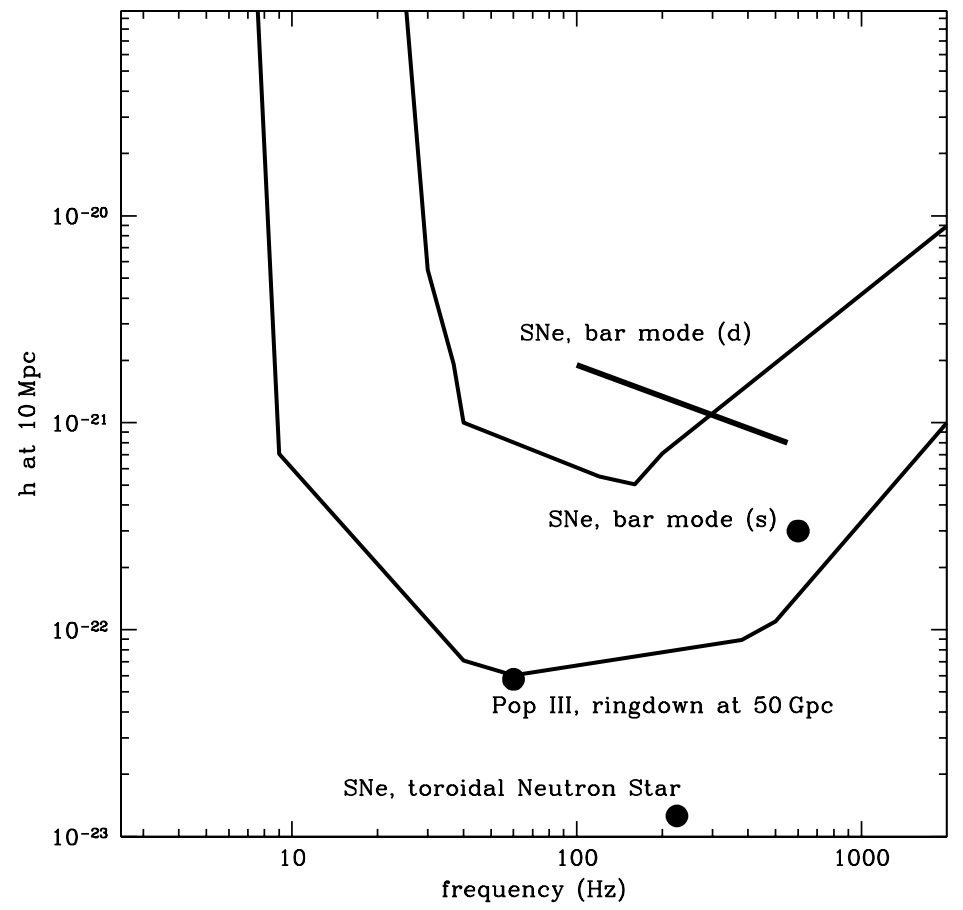

Figure 23: A comparison between the GW amplitude $h(f)$ for various sources and the LIGO-II sensitivity curve based on the analytic estimates from FHH [106]. See the text for details regarding the computations of $h$. The SNe sources at $10 \mathrm{Mpc}$; and the Population III sources at a luminosity distance of $\sim 50 \mathrm{Gpc}$. Secular bar-mode sources are identified with an (s), dynamical bar modes with a (d). This assumes strong bar modes exist, which has not been demonstrated robustly.

FHH [106] (and [108]) compute an upper limit (via Equation (9)) to the emitted amplitude from their dynamically unstable model of $h \sim 3 \times 10^{-22}$ (if coherent emission from a bar located at $10 \mathrm{Mpc}$ persists for 100 cycles). The corresponding frequency and maximum power are $f_{\mathrm{GW}} \approx$ $10^{3} \mathrm{~Hz}$ and $P_{\mathrm{GW}}=10^{53} \mathrm{erg} \mathrm{s}^{-1}$. LIGO-II should be able to detect such a signal (see Figure 23, where FHH's upper limit to $h$ for this dynamical bar mode is identified). 
The GW emission from proto neutron stars that are secularly unstable to the bar mode has been examined by Lai and Shapiro $[173,171]$. They predict that such a bar located at $10 \mathrm{Mpc}$ would emit GWs with a peak characteristic amplitude $h \sim 10^{-21}$, if the bar persists for $10^{2}-10^{4}$ cycles. The maximum $f_{\mathrm{GW}}$ of the emitted radiation is in the range of $10^{2}-10^{3} \mathrm{~Hz}$. This type of signal should be easily detected by LIGO-I (although detection may require a technique like the fast chirp transform method of Jenet and Prince [157], due to the complicated phase evolution of the emission). Ou et al. [236] found that a bar instability was maintained for several orbits before sheer flows disintegrate the instability, producing GW emissions that would have a signal-to-noise ratio greater than 8 for LIGO-II out to $32 \mathrm{Mpc}$. A movie of this simulation is shown in Figure 24.

Many stellar models models (e.g., [135, 138]) do not produce stars with sufficiently high spin rates to produce fast-rotating cores [245, 103, 113, 325, 231, 69]. Fryer \& Heger [103] argued that the explosion phase could eject a good deal of low angular-momentum material along the poles in their evolutions. Roughly $1 \mathrm{~s}$ after the collapse, the angular momentum in the faster cores will exceed the secular bar instability limit. It is also possible that standard stellar evolution models underestimate the rotation rate. Many scenarios for GRBs require much higher rotation rates than these models predict. This requirement has led to a host of new models predicting much faster rotation rates: mixing in single stars [338, 340], binary scenarios [114, 240, 104, 319, 42], and the collaspe of merging white dwarfs [341, 339, 342, 118, 63, 61]. For example, Fryer \& Heger [104] merged helium stars in an effort to increase this angular momentum (Figure 9). For Population III $300 M_{\odot}$ stars, the situation may be better. Figure 25 shows the $\beta$ value and growth time for such a $300 M_{\odot}$ star at bounce and just prior to the collapse to a black hole. This bounce is caused by a combination of thermal and rotational support and hence the bounce is very soft. Fryer et al. [115] argued that bar modes could well develop in these systems.

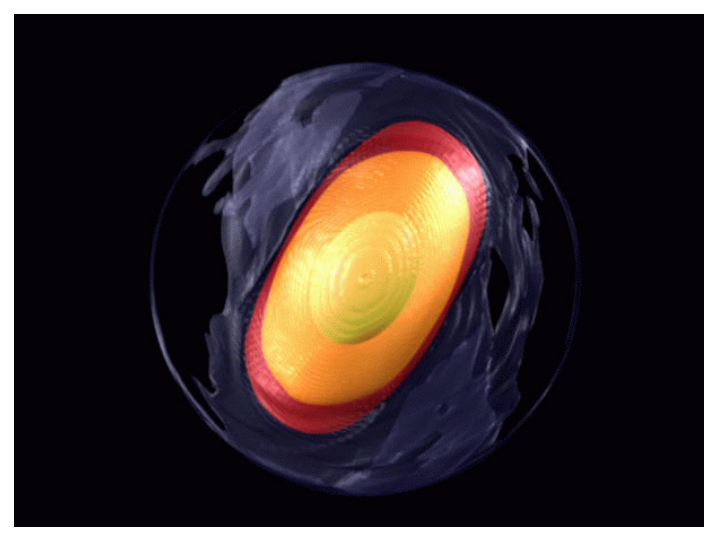

Figure 24: Still from a movie showing Evolution of a secular bar instability, see Ou et al. [236] for details. (To watch the movie, please go to the online version of this review article at http://www. livingreviews . org/lrr-2011-1.)

First, scientists have learned that dynamical instabilities can excite $m=1$ modes as well as the well-studied, $m=2$ bar modes [46, 258, 234, 235, 260]. Second, scientists have discovered that non-axisymmetric instabilities can occur at much lower values of $\beta$ when the differential rotation is very high $[278,279,282,326,257,260]$. For some models, cores with high differential rotation have exhibited non-axisymmetric instabilities for values of $\beta \approx 0.01$. These results may drastically change the importance of these modes in astrophysical observations. 


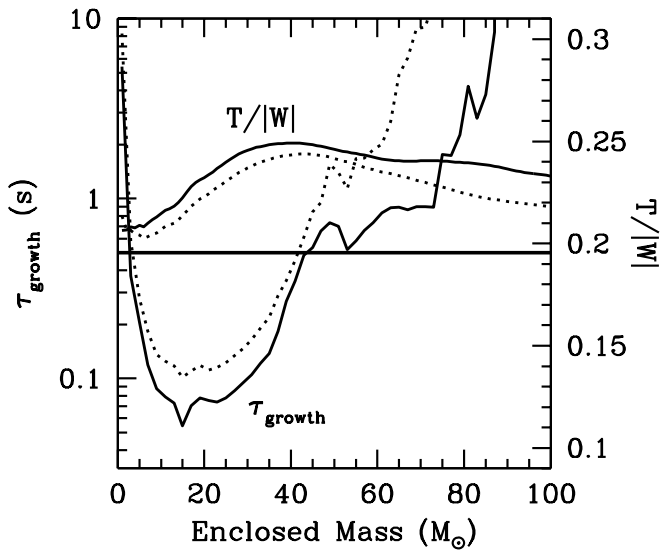

Figure 25: $\beta$ and instability growth time versus mass in the proto black hole formed in the collapse of a $300 M_{\odot}$ star at bounce (dotted line) and just prior to black-hole formation (solid line). Figure 10 of [115].

\subsubsection{Equilibrium models to study instabilities}

One way to determine whether a specific star collapses to develop bar modes is through equilibrium models as initial conditions for hydrodynamical simulations (e.g., [289, 237, 221, 46]). Such simulations represent the approximate evolution of a model beginning at some intermediate phase during collapse or the evolution of a collapsed remnant. These studies do not typically follow the intricate details of the collapse itself. Instead, their goals include determining the stability of models against the development of non-axisymmetric modes and estimation of the characteristics of any resulting GW emission.

Liu and Lindblom $[184,183]$ have applied this equilibrium approach to AIC. Their investigation began with a study of equilibrium models built to represent neutron stars formed from AIC [184]. These neutron star models were created via a two-step process, using a Newtonian version of Hachisu's self-consistent field method [128]. Hachisu's method ensures that the forces due to the centrifugal and gravitational potentials and the pressure are in balance in the equilibrium configuration.

Liu and Lindblom's process of building the nascent neutron stars began with the construction of rapidly-rotating, pre-collapse white dwarf models. Their Models I and II are C-O white dwarfs with central densities $\rho_{\mathrm{c}}=10^{10}$ and $6 \times 10^{9} \mathrm{~g} \mathrm{~cm}^{-3}$, respectively (recall this is the range of densities for which AIC is likely for C-O white dwarfs). Their Model III is an O-Ne-Mg white dwarf that has $\rho_{\mathrm{c}}=4 \times 10^{9} \mathrm{~g} \mathrm{~cm}^{-3}$ (recall this is the density at which collapse is induced by electron capture). All three models are uniformly rotating, with the maximum allowed angular velocities. The models' values of total angular momentum are roughly $3-4$ times that of Fryer et al.'s AIC progenitor Model 3 [102]. The realistic equation of state used to construct the white dwarfs is a Coulomb-corrected, zero temperature, degenerate gas equation of state [261, 51].

In the second step of their process, Liu and Lindblom [184] built equilibrium models of the collapsed neutron stars themselves. The mass, total angular momentum, and specific angular momentum distribution of each neutron-star remnant is identical to that of its white dwarf progenitor (see Section 3 of [184] for justification of the specific angular momentum conservation assumption). These models were built with two different realistic neutron-star equations of state.

Liu and Lindblom's cold neutron-star remnants had values of the stability parameter $\beta$ ranging from $0.23-0.26$. It is interesting to compare these results with those of Villain et al. [323] or of Zwerger and Müller [350]. Villain et al. [323] found maximum $\beta$ values of 0.2 for differentially- 
rotating models and 0.11 for rigidly-rotating models. Zwerger and Müller performed axisymmetric hydrodynamics simulations of stars with polytropic equations of state $\left(P \propto \rho^{\Gamma}\right)$. Their initial models were $\Gamma=4 / 3$ polytropes, representative of massive white dwarfs. All of their models started with $\rho_{\mathrm{c}}=10^{10} \mathrm{~g} \mathrm{~cm}^{-3}$. Their model that was closest to being in uniform rotation (A1B3) had $22 \%$ less total angular momentum than Liu and Lindblom's Model I. The collapse simulations of Zwerger and Müller that started with model A1B3 all resulted in remnants with values of $\beta<0.07$. Comparison of the results of these two studies could indicate that the equation of state may play a significant role in determining the structure of collapsed remnants. Or it could suggest that the assumptions employed in the simplified investigation of Liu and Lindblom are not fully appropriate.

In a continuation of the work of Liu and Lindblom, Liu [183] used linearized hydrodynamics to perform a stability analysis of the cold neutron-star AIC remnants of Liu and Lindblom [184]. He found that only the remnant of the O-Ne-Mg white dwarf (Liu and Lindblom's Model III) developed the dynamical bar mode $(m=2)$ instability. This model had an initial $\beta=0.26$. Note that the $m=1$ mode, observed by others to be the dominant mode in unstable models with values of $\beta$ much lower than 0.27 [314, 332, 237, 46], did not grow in this simulation. Because Liu and Lindblom's Models I and II had lower values of $\beta$, Liu identified the onset of instability for neutron stars formed via AIC as $\beta_{\mathrm{d}} \approx 0.25$.

Liu estimated the peak amplitude of the GWs emitted by the Model III remnant to be $h_{\mathrm{pk}} \approx$ $1.4 \times 10^{-24}$ and the LIGO-II signal-to-noise ratio (for a persistent signal like that seen in the work of [221] and [30]) to be $S / N \leq 3$ (for $f_{\mathrm{GW}} \approx 450 \mathrm{~Hz}$ ). These values are for a source located at $100 \mathrm{Mpc}$. He also predicted that the timescale for gravitational radiation to carry away enough angular momentum to eliminate the bar mode is $\tau_{\mathrm{GW}} \approx 7 \mathrm{~s}\left(\sim 3 \times 10^{3}\right.$ cycles $)$. Thus, $h \approx 8 \times 10^{-23}$. (Note that this value for $h$ is merely an upper limit as it assumes that the amplitude and frequency of the GWs do not change over the $7 \mathrm{~s}$ during which they are emitted. Of course, they will change as angular momentum is carried away from the object via GW emission.) Such a signal may be marginally detectable with LIGO-II (see Figure 23). Details of the approximations on which these estimates are based can be found in [183].

Liu cautions that his results hold if the magnetic field of the proto neutron star is $B \leq 10^{12} \mathrm{G}$. If the magnetic field is larger, then it may have time to suppress some of the neutron star's differential rotation before it cools. This would make bar formation less likely. Such a large field could only result if the white dwarf progenitor's $B$ field was $\geq 10^{8} \mathrm{G}$. Observation-based estimates suggest that about $25 \%$ of white dwarfs in interacting close binaries (cataclysmic variables) are magnetic and that the field strengths for these stars are $\sim 10^{7}-3 \times 10^{8} \mathrm{G}$ [330]. Strong toroidal magnetic fields of $B \geq 10^{14} \mathrm{G}$ will also suppress the bar instabilities [119].

\subsubsection{Hydrodynamic models}

The GW emission from non-axisymmetric hydrodynamics simulations of stellar collapse was first studied by Bonazzola and Marck [194, 23]. They used a Newtonian, pseudo-spectral hydrodynamics code to follow the collapse of polytropic models. Their simulations covered only the pre-bounce phase of the collapse. They found that the magnitudes of $h_{\mathrm{pk}}$ in their 3D simulations were within a factor of two of those from equivalent $2 \mathrm{D}$ simulations and that the gravitational radiation efficiency did not depend on the equation of state.

The first use of 3D hydrodynamics collapse simulations to study the GW emission well beyond the core bounce phase was performed by Rampp, Müller, and Ruffert [245]. These authors started their Newtonian simulations with the only model (A4B5G5) of Zwerger and Müller [350] that had a post-bounce value for the stability parameter $\beta=0.35$ that significantly exceeded 0.27 (recall this is the value at which the dynamical bar instability sets in for MacLaurin spheroid-like models). This model had the softest equation of state $\left(\Gamma_{\mathrm{r}}=1.28\right)$, highest $\beta_{\mathrm{i}}=0.04$, and largest degree of 
differential rotation of all of Zwerger and Müller's models. The model's initial density distribution had an off-center density maximum (and therefore a torus-like structure). Rampp, Müller, and Ruffert evolved this model with a $2 \mathrm{D}$ hydrodynamics code until its $\beta$ reached $\sim 0.1$. At that point, 2.5 ms prior to bounce, the configuration was mapped onto a 3D nested cubical grid structure and evolved with a 3D hydrodynamics code.

Before the 3D simulations started, non-axisymmetric density perturbations were imposed to seed the growth of any non-axisymmetric modes to which the configuration was unstable. When the imposed perturbation was random ( $5 \%$ in magnitude), the dominant mode that arose was $m=4$. The growth of this particular mode was instigated by the cubical nature of the computational grid. When an $m=3$ perturbation was imposed (10\% in magnitude), three clumps developed during the post-bounce evolution and produced three spiral arms. These arms carried mass and angular momentum away from the center of the core. The arms eventually merged into a bar-like structure (evidence of the presence of the $m=2$ mode). Significant non-axisymmetric structure was visible only within the inner $40 \mathrm{~km}$ of the core. Their simulations were carried out to $\sim 14 \mathrm{~ms}$ after bounce.

The amplitudes of the emitted gravitational radiation (computed in the quadrupole approximation) were only $\sim 2 \%$ different from those observed in the 2D simulation of Zwerger and Müller. Because of low angular resolution in the 3D runs, the energy emitted was only $65 \%$ of that emitted in the corresponding $2 \mathrm{D}$ simulation.

The findings of Centrella et al. [46] indicate it is possible that some of the post-bounce configurations of Zwerger and Müller, which have lower values of $\beta$ than the model studied by Rampp, Müller, and Ruffert [245], may also be susceptible to non-axisymmetric instabilities. Centrella et al. have performed 3D hydrodynamics simulations of $\Gamma=1.3$ polytropes to test the stability of configurations with off-center density maxima (as are present in many of the models of Zwerger and Müller [350]). The simulations carried out by Centrella and collaborators were not full collapse simulations, but rather began with differentially-rotating equilibrium models. These simulations tracked the growth of any unstable non-axisymmetric modes that arose from the initial $1 \%$ random density perturbations that were imposed. Their results indicate that such models can become dynamically unstable at values of $\beta \gtrsim 0.14$. The observed instability had a dominant $m=1$ mode. Centrella et al. estimate that if a stellar core of mass $M \sim 1.4 M_{\odot}$ and radius $R \sim 200 \mathrm{~km}$ encountered this instability, the values of $h_{\mathrm{pk}}$ from their models would be $\sim 2 \times 10^{-24}-2 \times 10^{-23}$, for $d=10 \mathrm{Mpc}$. The frequency at which $h_{\mathrm{pk}}$ occurred in their simulations was $\sim 200 \mathrm{~Hz}$. This instability would have to persist for at least $\sim 15$ cycles to be detected with LIGO-II.

Brown [31] carried out an investigation of the growth of non-axisymmetric modes in postbounce cores that was similar in many respects to that of Rampp, Müller, and Ruffert [245]. He performed 3D hydrodynamical simulations of the post-bounce configurations resulting from 2D simulations of core collapse. His pre-collapse initial models are $\Gamma=4 / 3$ polytropes in rotational equilibrium. The differential rotation laws used to construct Brown's initial models were motivated by the stellar evolution study of Heger, Langer, and Woosley [137]. The angular velocity profiles of their pre-collapse progenitors were broad and Gaussian-like. Brown's initial models had peak angular velocities ranging from 0.8 -2.4 times those of [137]. The model evolved by Rampp, Müller, and Ruffert [245] had much stronger differential rotation than any of Brown's models. To induce collapse, Brown reduced the adiabatic index of his models to $\Gamma=1.28$, the same value used by [245].

Brown found that $\beta$ increased by a factor $\lesssim 2$ during his $2 \mathrm{D}$ collapse simulations. This is much less than the factor of $\sim 9$ observed in the model studied by Rampp, Müller, and Ruffert [245]. This is likely a result of the larger degree of differential rotation in the model of Rampp et al.

Brown performed 3D simulations of the two most rapidly-rotating of his post-bounce models (models $\Omega 24$ and $\Omega 20$, both of which had $\beta>0.27$ after bounce) and of the model of Rampp et al. (which, although it starts out with $\beta=0.35$, has a sustained $\beta<0.2$ ). Brown refers to the Rampp et al. model as model RMR. Because Brown's models do not have off-center density maxima, they 
are not expected to be unstable to the $m=1$ mode observed by Centrella et al. [46]. He imposed random $1 \%$ density perturbations at the start of all three of these 3D simulations (note that this perturbation was of a much smaller amplitude than those imposed by [245]).

Brown's simulations determined that both his most rapidly-rotating model $\Omega 24$ (with postbounce $\beta>0.35$ ) and model RMR are unstable to growth of the $m=2$ bar mode. However, his model $\Omega 20$ (with post-bounce $\beta>0.3$ ) was stable. Brown observed no dominant $m=3$ or $m=4$ modes growing in model RMR at the times at which they were seen in the simulations of Rampp et al. This suggests that the mode growth in their simulations was a result of the large perturbations they imposed. The $m=2$ mode begins to grow in model RMR at about the same time as Rampp et al. stopped their evolutions. No substantial $m=1$ growth was observed.

The results of Brown's study indicate that the overall $\beta$ of the post-bounce core may not be a good diagnostic for the onset of instability. He found, as did Rampp, Müller, and Ruffert [245], that only the innermost portion of the core (with $\rho>10^{10} \mathrm{~g} \mathrm{~cm}^{-3}$ ) is susceptible to the bar mode. This is evident in the stability of his model $\Omega 20$. This model had an overall $\beta>0.3$, but an inner core with $\beta_{\text {ic }}=0.15$. Brown also observed that the $\beta$ of the inner core does not have to exceed 0.27 for the model to encounter the bar mode. Models $\Omega 24$ and RMR had $\beta_{\mathrm{ic}} \approx 0.19$. He speculates that the inner cores of these later two models may be bar-unstable because interaction with their outer envelopes feeds the instability or because $\beta_{\mathrm{d}}<0.27$ for such configurations.

The GW emission from non-radial quasinormal mode oscillations in proto neutron stars has been examined by Ferrari, Miniutti, and Pons [85]. They found that the frequencies of emission $f_{\mathrm{GW}}$ during the first second after formation $(600-1100 \mathrm{~Hz}$ for the first fundamental and gravity modes) are significantly lower than the corresponding frequencies for cold neutron stars and thus reside in the bandwidths of terrestrial interferometers. However, for first generation interferometers to detect the GW emission from an oscillating proto neutron star located at $10 \mathrm{Mpc}$, with a signalto-noise ratio of $5, E_{\mathrm{GW}}$ must be $\sim 10^{-3}-10^{-2} M_{\odot} c^{2}$. It is unlikely that this much energy is stored in these modes (the collapse itself may only emit $\sim 10^{-7} M_{\odot} c^{2}$ in gravitational waves [67]).

Shibata et al. [278, 279] found that, with extremely differentially-rotating cores, a bar mode instability can occur at $\beta$ values of 0.01 . They found that such an instability was weakly dependent on the polytropic index describing the equation of state and on the velocity profile (as long as the differential rotation is high). They predict an effective amplitude of roughly $10^{-22}$ at a distance of $100 \mathrm{Mpc}$.

Studies of systems with extreme differential rotation have also discovered the development of one-arm $(m=1)$ instabilities. The work of Centrella et al. [46] has been followed by a large set of results, varying the density and angular velocity profiles [46, 258, 234, 235, 260]. Ou \& Tohline [235] argued that these instabilities are akin to the Rossby wave instabilities studied in black-hole accretion disks [176]. These one-armed spirals could also produce a considerable GW signal.

Watts et al. [326] argue that these low- $\beta$ instabilities can be explained if the corotating $\mathrm{f}$ mode develops a dynamical shear instability. This occurs when the corotating f-mode enters the corotation band and when the degree of differential rotation exceeds a threshold value (that is within those produced in some collapse progenitors). These new instabilities are drastically changing our view of non-axisymmetric modes. 


\subsection{Neutrinos}

Up until now, we have focused on the GWs from a changing quadrupole moment of the baryonic matter. Anisotropic neutrino emission may also produce GWs. Although less studied than baryonic motions, GW emission from aniostropic neutrino emission [76, 317] has been investigated in proto neutron star convection, convection above the proto neutron star, and in asymmetric collapse (caused either by asymmetries in the collapse or by rotation).

Müller et al. [209] have modeled the emission of GWs from anisotropic neutrino emission from convection both inside and above the proto neutron star for non-rotating models. The signal from the proto neutron star is shown in Figure 26. Note that although the quadrupole amplitude for the neutrino emission is much higher, it varies slower than the mass motions. Hence, it only dominates the signal at low frequencies. This model is of a cooling, isolated proto neutron star that developed much stronger convection than is seen in proto neutron star models of full systems. As such, it represents an upper limit for the GW signal of non-rotating proto neutron stars. Figure 26 shows the signal for the convection above the proto neutron star. Like in the case of neutrino emission for proto neutron star convection, anisotropic neutrino emission from convection above the proto neutron star dominates the low-frequency part of the signal.

For mass motions, rotation strongly increases the signal at bounce and during the convective phase. But the neutrino signal does not increase as dramatically. Kotake et al. [166] found that the signal from a rotating collapse is dominated at nearly all frequencies by the matter contribution (Figure 27). This is because the neutrino signal is axially symmetric and the variation in the quadrupole moment is fairly weak. However, within the SASI paradigm, Kotake et al. [165] found that the amplitude of the GW signal is two orders of magnitude larger than those from convective matter motions outside the proto neutron star. This result has been confirmed by several groups [195, 228].

The asymmetric collapse simulations discussed in Section 4.2 are one way to increase the GW signal from anisotropic neutrino emission. Recall from Figure 17 that Burrows \& Hayes found that the neutrino-induced term dominated the GW signal for their asymmetric collapse simulations. Fryer et al. [107] found the same result. The GW amplitude is dominated by the neutrino component and can exceed $h_{\mathrm{pk}} \sim 6 \times 10^{-24}$, for a source located at $10 \mathrm{Mpc}$ in Fryer's most extreme example. A caveat in this result is that Fryer et al. were artificially increasing the level of the asymmetry in the collapse in an attempt to obtain strong neutron star kicks and it is unlikely that any stellar system will have such large asymmetries in nature. This signal estimate should be seen as an extreme upper limit.

Anisotropic neutrino emission has several distinguishing features that will allow us to differentiate it from a matter-driven GW signal. It has much less variation in the time structure and will produce a stronger signal at lower frequencies. But much more work must be done to understand fully the neutrino-driven GW signal. For example, GWs can be produced by asymmetric neutrino emission from the core (e.g., from oscillations with sterile neutrinos [187]). If magnetic fields induce asymmetries in this oscillation and the neutron star is rotating, the sterile neutrino emission can produce a neutrino-driven GW signature that is very different from what was shown here. 

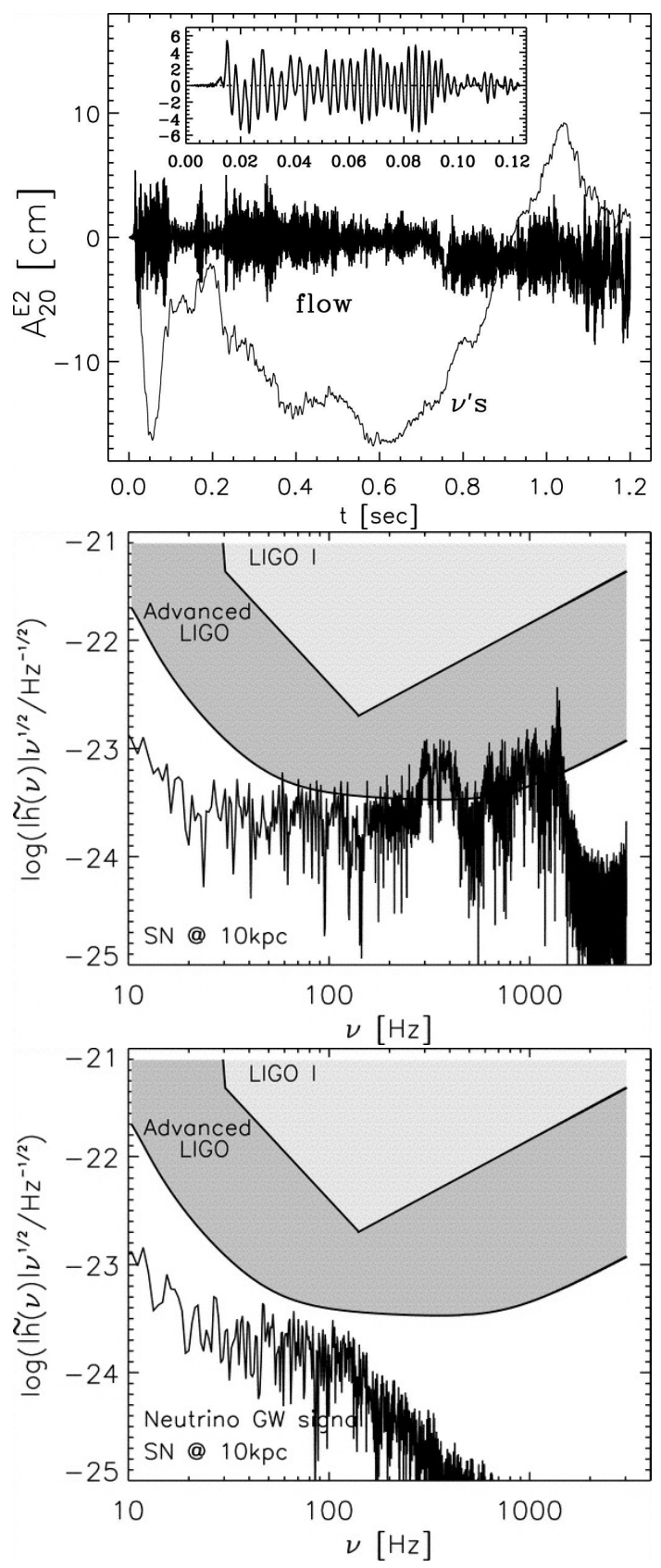

Figure 26: The GW signal from convection in a proto neutron star. The top panel shows the GW quadrupole amplitude $A_{20}^{\mathrm{E} 2}$ as a function of time for both the convective mass flow (thick line) and anisotropic neutrino emission (thin line). The combined spectral energy distribution of the quadrupole radiation for both sources is shown in the middle figure, with just the neutrino component alone in the bottom panel. Note that although the quadrupole amplitude for the neutrino emission is much higher, it varies slower than the mass motions. Hence, it only dominates the signal at low frequencies. (Figures from [209]; used with permission.) 

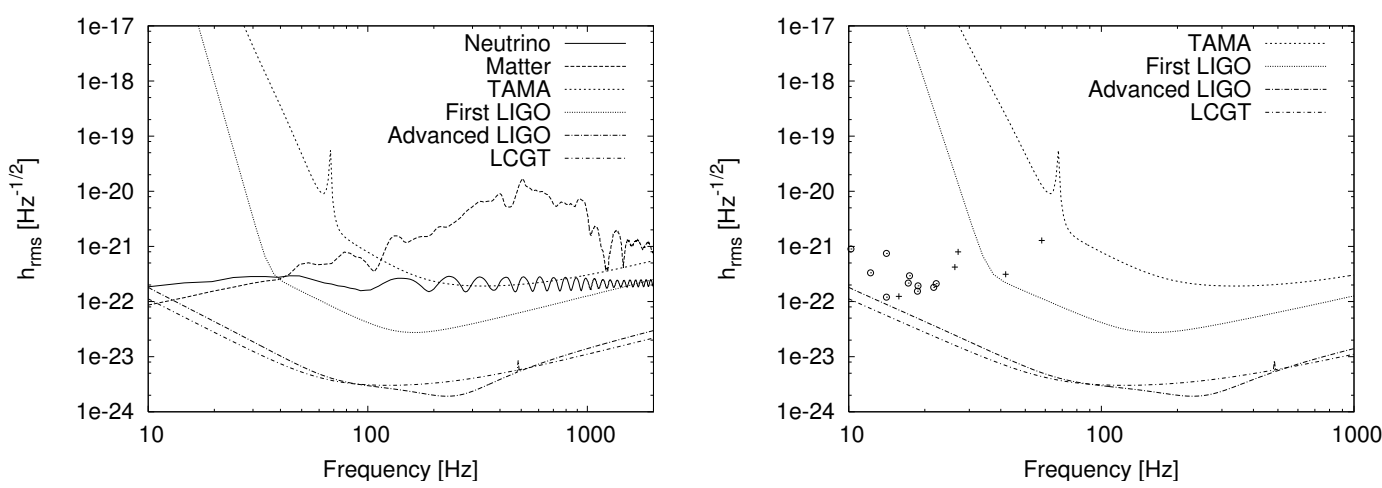

Figure 27: Detection limits of TAMA, first LIGO, advanced LIGO, and Large-scale Cryogenic Gravitational wave Telescope (LCGT) with the expected GW spectrum obtained from the numerical simulations. The left panel shows the GW spectrum contributed from neutrinos (solid) and from the matter (dashed) in a rotating model with $\Omega=4 \mathrm{rad} \mathrm{s}^{-1}$ imposed initially on a $15 M_{\odot}$ progenitor model. In the right panel, the open circles and the pluses represent the amplitudes of $h_{\nu, \text { eq }}$ with the characteristic frequencies of $\nu_{\mathrm{eq}}$ for the models with the cylindrical and the shell-type rotation profiles, respectively. Under the frequency of $\nu_{\mathrm{eq}}$, the GWs from the neutrinos dominate over those from the matter contributions. From the panel, it is seen that the GWs from neutrinos dominate over the ones from the matter in a lower frequency $(f \leq 100 \mathrm{~Hz})$. Note that the source is assumed to be located at the distance of $10 \mathrm{kpc}$. (Figures from [166]; used with permission.)
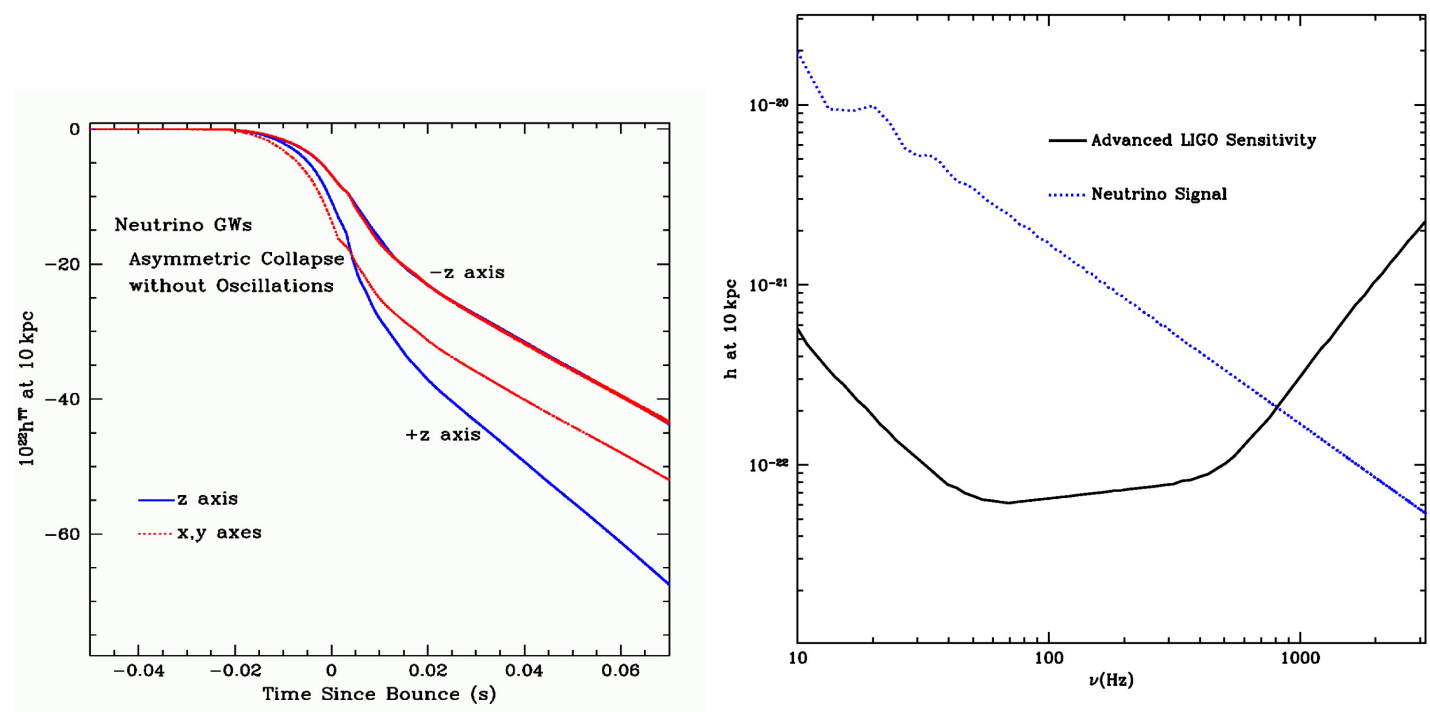

Figure 28: The gravitational waveform for neutrino contributions from the asymmetric collapse simulations of Fryer et al. [107]. The curves plot the product of the GW amplitude to the source as a function of time. (Figures from [107]; used with permission.) 


\section{$4.5 r$-Modes}

$r$-modes are quasitoroidal oscillations in rotating fluids that occur because of the Coriolis effect (akin to the Rossby modes studied in planetary "spots"). In GWs, these modes are driven unstable by gravitational radiation reaction. The primary questions surrounding these instabilities arise in calculating the saturation amplitude of $r$-modes. The GW emission from $r$-mode unstable neutronstar remnants of core-collapse SNe would be easily detectable if $\alpha_{\max } \sim 1$. In rotating stars, gravitational radiation reaction drives the $r$-modes toward unstable growth $[4,96]$. In hot, rapidlyrotating neutron stars, this instability may not be suppressed by internal dissipative mechanisms (such as viscosity and magnetic fields) [179]. If not limited, the dimensionless amplitude $\alpha$ of the dominant $(m=2) r$-mode will grow to order unity within ten minutes of the formation of a neutron star rotating with a millisecond period. The emitted GWs carry away angular momentum, and will cause the newly-formed neutron star to spin down over time. The spin-down timescale and the strength of the GWs themselves are directly dependent on the maximum value $\alpha_{\max }$ to which the amplitude is allowed to grow [180, 181].

Originally, it was thought that $\alpha_{\max } \sim 1$. In such a case, we can estimate the GW signal from stellar collapse. We expect multiple GW bursts to occur as material falls back onto the neutron star and results in repeat episodes of $r$-mode growth (note that a single $r$-mode episode can have multiple amplitude peaks [180]). Using Equation (10), FHH [106] calculate that the characteristic amplitude of the GW emission from this $r$-mode evolution tracks from $6-1 \times 10^{-22}$, over a frequency range of $10^{3}-10^{2} \mathrm{~Hz}$ for a source driven by fallback at $10 \mathrm{Mpc}$. They estimate the emitted energy to exceed $10^{52} \mathrm{erg}$.

Later work indicated that $\alpha_{\max }$ may be $\geq 3$ [180, 294, 268, 181]. But most recent research suggests that magnetic fields, hyperon cooling, and hyperon bulk viscosity may limit the growth of the $r$-mode instability, even in nascent neutron stars [159, 158, 248, 249, 181, 178, 132, 5] (significant uncertainties remain regarding the efficacy of these dissipative mechanisms). One way to reduce this viscosity is to invoke non-standard physics in the dense equation of state, e.g., quark material, anti-kaon core [53, 71]. Even with this more exotic physics, the reduction in viscosity is limited to specific regions in the neutron star and in the spin/temperature phase space. More work is needed to determine if such modifications can allow $r$-modes to make a detectable signal.

In addition, a study of a simple barotropic neutron star model by Arras et al. [8] argue that multimode couplings could limit $\alpha_{\max }$ to values $\ll 1$. If $\alpha_{\max }$ is indeed $\ll 1$ (see also [125, 27, 26, 28, 252, 24]), GW emission from $r$-modes in collapsed remnants is likely undetectable. From Equation (10), we can see that the GW signal is proportional to the mode amplitude, so a decrease in the maximum amplitude by an order of magnitude corresponds to an order of magnitude decrease in the GW strain. If correct, and much of the community believes the maximum of the mode amplitude may be even smaller than 0.1, the GW signal from $r$-modes is much lower than any other GW source and will not contribute significantly to the observed signal in stellar collapse. Because of this, $r$-mode sources are omitted from figures comparing source strengths and detector sensitivities and from discussions of likely detectable sources in the concluding section.

$r$-modes may still produce signals in accreting systems such as low-mass X-ray binaries. Work continues in this subject for these systems, but this is beyond the subject of this review. 


\subsection{Fragmentation}

Fragmentation requires even more extreme spin rates than bar-mode instabilities. It is unlikely that any neutron-star-forming systems will have enough rotation to produce fragmentation. For most stellar models, the angular momentum increases with radius (or enclosed mass). When black holes form, material further out in the star (with higher angular momentum) becomes part of the compact remnant and it is possible to form proto black holes with higher spin rates than proto neutron stars. These proto black holes may be more likely to fragment. Even so, fragmentation requires extreme levels of rotation. As we shall see, some cases of GW emission from fragmentation is intimately related to black-hole ringing and some of the GW sources discussed here will also be discussed in Section 4.7.

Duez et al. [73] found that if a black hole does form, but the disk is spinning rapidly, that the disk will fragment and its subsequent accretion will be in spurts, causing a "splash" onto the black hole, producing ringing and GW emission. Their result implies very strong GW amplitudes $\gtrsim 10^{-21}$ at distances of $10 \mathrm{Mpc}$. Black hole ringing was also estimated by FHH [106], where they too assumed discrete accretion events. They found that, even with very optimistic accretion scenarios, such radiation will be of very low amplitude and beyond the upper frequency reach of LIGO-II (see [106] for details).

The general-relativistic hydrodynamics simulations of Zanotti, Rezzolla, and Font [344] suggest that a torus of neutron-star matter surrounding a black-hole remnant may be a stronger source of GWs than the collapse itself. They used a high-resolution shock-capturing hydrodynamics method in conjunction with a static (Schwarzschild) spacetime to follow the evolution of "toroidal neutron stars". Their results indicate that if a toroidal neutron star (with constant specific angular momentum) is perturbed, it could undergo quasi-periodic oscillations. They estimate that the resulting GW emission would have a characteristic amplitude $h_{\mathrm{c}}$ ranging from $6 \times 10^{-24}-5 \times 10^{-23}$, for ratios of torus mass to black-hole mass in the range $0.1-0.5$. (These amplitude values are likely underestimated because the simulations of Zanotti et al. are axisymmetric.) The corresponding frequency of emission is $f_{\mathrm{GW}} \approx 200 \mathrm{~Hz}$. The values of $h_{\mathrm{c}}$ and $f_{\mathrm{GW}}$ quoted here are for a source located at $10 \mathrm{Mpc}$. This emission would be just outside the range of LIGO-II (see Figure 23). Further numerical investigations, which study tori with non-constant angular momenta and include the effects of self-gravity and black hole rotation, are needed to confirm these predictions. Movies from the simulations of Zanotti et al. can be viewed at [247].

Magnetized tori around rapidly-spinning black holes (formed either via core collapse or neutronstar-black-hole coalescence) have been examined in the theoretical study of van Putten and Levinson [321]. They find that such a torus-black-hole system can exist in a suspended state of accretion if the ratio of poloidal magnetic-field energy to kinetic energy $E_{B} / E_{\mathrm{k}}$ is less than 0.1 . They estimate that $\sim 10 \%$ of the spin energy of the black hole will be converted to gravitational-radiation energy through multipole mass-moment instabilities that develop in the torus. If a magnetized torus-black-hole system located at $10 \mathrm{Mpc}$ is observed for $2 \times 10^{4}$ rotation periods, the characteristic amplitude of the GW emission is $\sim 6 \times 10^{-20}$. It is possible that this emission could take place at several frequencies. Observations of x-ray lines from GRBs (which are possibly produced by these types of systems) could constrain these frequencies by providing information regarding the angular velocities of the tori: preliminary estimates from observations suggest $f_{\mathrm{GW}} \approx 500 \mathrm{~Hz}$, placing the radiation into a range detectable by LIGO-I [321].

By studying the results of current stellar-collapse models, FHH [106] predict that a fragmentation instability is unlikely to develop during core-collapse SNe because the cores have central density maxima (see also [108]). However, they do give estimates [calculated via Equations (7) and (6)] for the amplitude, power, and frequency of the emission from such an instability: $h_{\mathrm{pk}} \approx 2 \times 10^{-22}$, $P_{\mathrm{GW}}=10^{54} \mathrm{erg} \mathrm{s}^{-1}, f_{\mathrm{GW}} \approx 2 \times 10^{3} \mathrm{~Hz}$. Again, this signal would fall just beyond the upper limit of LIGO-II's frequency range. 
Three-dimensional models of black-hole formation in a rotating stellar collapse are needed to truly study fragmentation. Zink et al. [348, 349] have found that with appropriate initial conditions, fragmentation can occur with even modest values of $T /|W|: \sim 0.2$. But for current stellar models, fragmentation remains difficult to achieve. Rockefeller et al. [250] modeled in three dimensions the collapse of a $60 M_{\odot}$ star using a range of angular momenta to study the effects of the spin on the GW signal. For moderate spin rates, the instabilties in the disk grow and form pockets of denser material, but strong fragmentation does not occur (Figure 29). The GW signal is far lower than the upper limits from FHH or those predicted by van Putten. Liu et al. [185] have performed axisymmetric calculations of the collapse of an SMS using a magneto-hydrodynamic code. As this group moves to 3 -dimensional calculations, we will be able to test the development of these instabilities within strong magnetic fields.

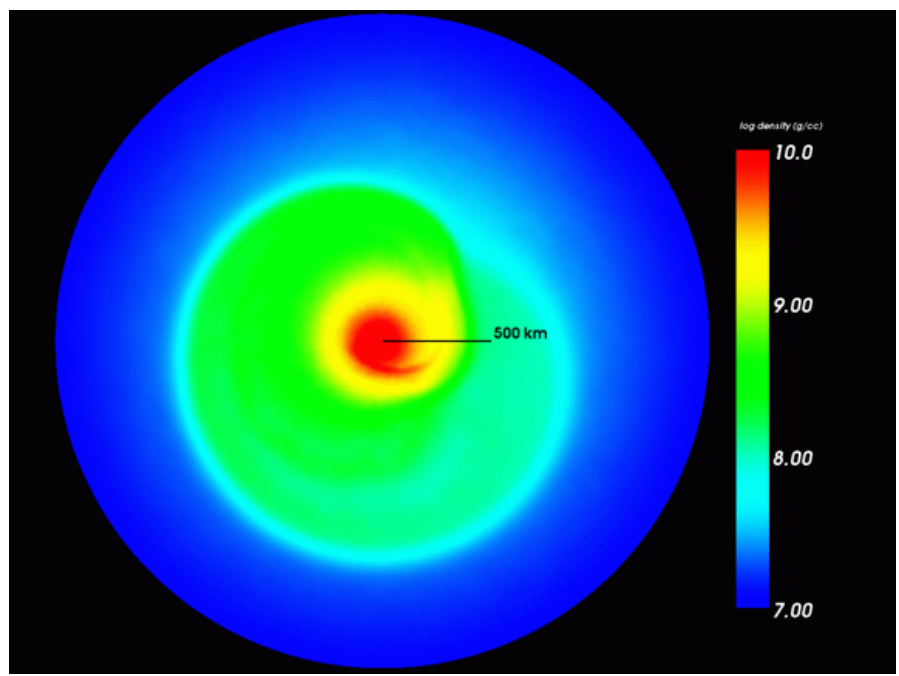

Figure 29: The matter density in the equatorial plane of the rapidly-rotating collapsar simulation, $0.44 \mathrm{~s}$ after collapse. The spiral wave forms near the center of the collapsar $0.29 \mathrm{~s}$ after collapse and moves outward through the star. (Figure 4 of [250]; used with permission.) 


\subsection{Ringing}

Most studies of quasi-normal modes in black holes (black-hole ringing) have focused on the collapse of SMSs. A number of reviews already exist on this topic $[162,18,17,84]$ and we refer the reader to these texts for more details. For stellar-massed black holes, ringing is intimately connected to fragmentation. As we discussed in Section 4.6, although Duez et al. [73] found that fragmentation could lead to black-hole ringing with very strong GW amplitudes $\gtrsim 10^{-21}$ at distances of $10 \mathrm{Mpc}$, FHH found that even with very optimistic accretion scenarios, such radiation will be of very low amplitude and beyond the upper frequency reach of LIGO-II (see [106] for details). In numerical studies, Nagar et al. [215, 216] argue that this accretion is not a superposition of quasi-normal modes (although see [9], indicating that the signal would be even lower than simple analytic estimates might predict). The strongest black-hole ringing signal will occur in SMBHs and the rest of this section will focus on the GW signal from these objects.

The outcome of SMS collapse can be determined only with numerical, relativistic 3D hydrodynamics simulations. Until recently, such simulations had been published only for nearly spherical collapse. The spherical simulations of Shapiro and Teukolsky [275] produced collapse evolutions that were nearly homologous. In this case, the collapse time $\tau_{\text {coll }}$ is roughly the free-fall time at the horizon

$$
\tau_{\text {coll }}=\left(\frac{R^{3}}{4 \pi M}\right)^{1 / 2}=14 \mathrm{~s}\left(\frac{M}{10^{6} M_{\odot}}\right)^{-1} .
$$

The peak GW frequency $f_{\mathrm{GW}}=\tau_{\text {coll }}^{-1}$ is then $10^{-2} \mathrm{~Hz}$, if the mass of the star is $10^{6} M_{\odot}$. This is in the middle of LISA's frequency band of $10^{-4}-1 \mathrm{~Hz}[308,95]$.

The amplitude $h$ of this burst signal can be roughly estimated in terms of the star's quadrupole moment

$$
\begin{aligned}
h & \leq \epsilon \frac{2 M^{2}}{R d} \\
& \leq \epsilon \cdot 1 \times 10^{-18}\left(\frac{M}{10^{6} M_{\odot}}\right)\left(\frac{d}{50 \mathrm{Gpc}}\right)^{-1} .
\end{aligned}
$$

Here $d$ is the distance to the star and $\epsilon \sim T /|W|$ is a measure of the star's deviation from spherical symmetry. In this case, $\epsilon$ will be much less than one near the horizon, since the collapse is nearly spherical.

There are two possible aspherical collapse outcomes that have been studied. The first outcome is direct collapse to a SMBH. In this case, $\epsilon$ will be on the order of one near the horizon. Thus, according to Equation (31), the peak amplitude of the GW burst signal will be

$$
h_{\mathrm{pk}} \approx 1 \times 10^{-18}\left(\frac{M}{10^{6} M_{\odot}}\right)\left(\frac{d}{50 \mathrm{Gpc}}\right)^{-1} .
$$

Alternatively, the star may encounter the dynamical bar mode instability prior to complete collapse. Baumgarte and Shapiro [12] have estimated that a uniformly-rotating SMS will reach $\beta \approx 0.27$ when $R / M=15$. The frequency of the quasiperiodic gravitational radiation emitted by the bar can be estimated in terms of its rotation frequency to be

$$
\begin{aligned}
f_{\mathrm{GW}} & =2 f_{\mathrm{bar}} \sim 2\left(\frac{G M}{R^{3}}\right)^{1 / 2} \\
& =2 \times 10^{-3} \mathrm{~Hz}\left(\frac{M}{10^{6} M_{\odot}}\right)^{-1}
\end{aligned}
$$


when $R / M=15$. The corresponding $h_{\mathrm{pk}}$, again estimated in terms of the star's quadrupole moment, is

$$
\begin{aligned}
h_{\mathrm{pk}} & \leq \frac{2 M^{2}}{R d} \\
& \leq 1 \times 10^{-19}\left(\frac{M}{10^{6} M_{\odot}}\right)\left(\frac{d}{50 \mathrm{Gpc}}\right)^{-1} .
\end{aligned}
$$

The LISA sensitivity curve is shown in Figure 30 (see [149] for details on the computation of this curve; a mission time of three years has been assumed). The GW signal from this dynamical bar mode could be detected with LISA.

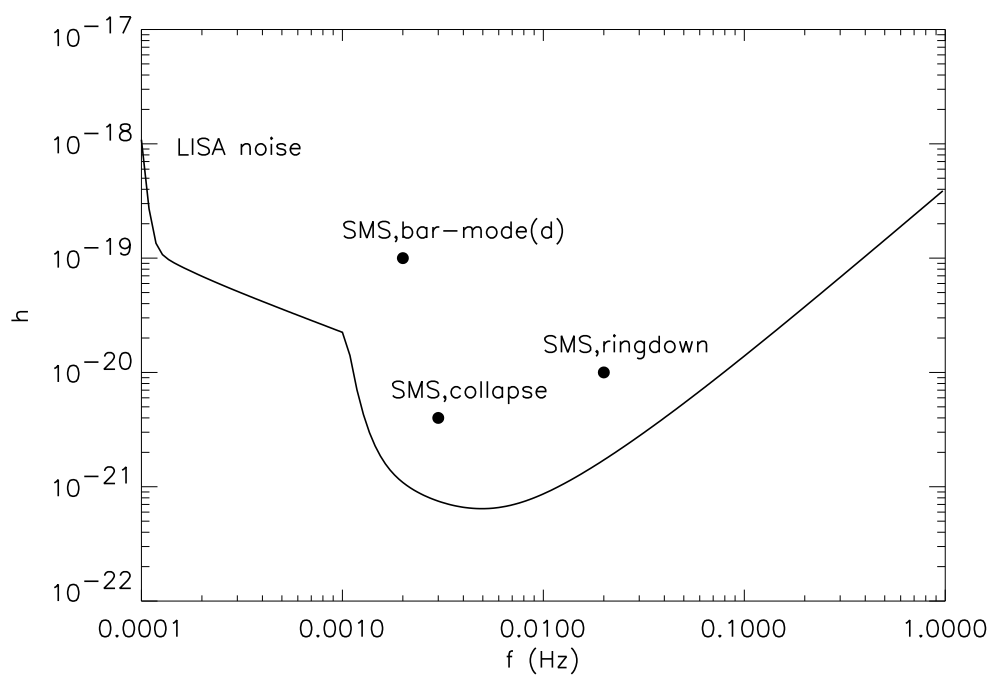

Figure 30: A comparison between the GW amplitude $h(f)$ for various sources and the LISA noise curve. See the text for details regarding the computations of $h$. The SMS sources are assumed to have masses of $\sim 10^{6} M_{\odot}$ and be located at a luminosity distance of $50 \mathrm{Gpc}$. The bar-mode source is a dynamical bar mode.

Shibata and Shapiro [284] have published a fully general-relativistic, axisymmetric simulation of the collapse of a rapidly, rigidly-rotating SMS. They found that the collapse remained homologous during the early part of the evolution. An apparent horizon does appear in their simulation, indicating the formation of a black hole. Because of the symmetry condition used in their run, non-axisymmetric instabilities did not develop.

The collapse of a uniformly-rotating SMS has been investigated with post-Newtonian hydrodynamics, in 3+1 dimensions, by Saijo, Baumgarte, Shapiro, and Shibata [259]. Their numerical scheme used a post-Newtonian approximation to the Einstein equations, but solved the fully relativistic hydrodynamics equations. Their initial model was an $n=3$ polytrope.

The results of Saijo et al. (confirmed in conformally-flat simulations [256]) indicate that the collapse of a uniformly-rotating SMS is coherent (i.e., no fragmentation instability develops). The collapse evolution of density contours from their model is shown in Figure 31. Although the work of Baumgarte and Shapiro [12] suggests that a bar instability should develop prior to black-hole formation, no bar development was observed by Saijo et al. They use the quadrupole approximation to estimate a mean GW amplitude from the collapse itself: $h=4 \times 10^{-21}$, for a $10^{6} M_{\odot}$ star located at a distance of $50 \mathrm{Gpc}$. Their estimate for $f_{\mathrm{GW}}$ at the time of black-hole formation is $3 \times 10^{-3} \mathrm{~Hz}$. This signal would be detectable with LISA (see Figure 30). 


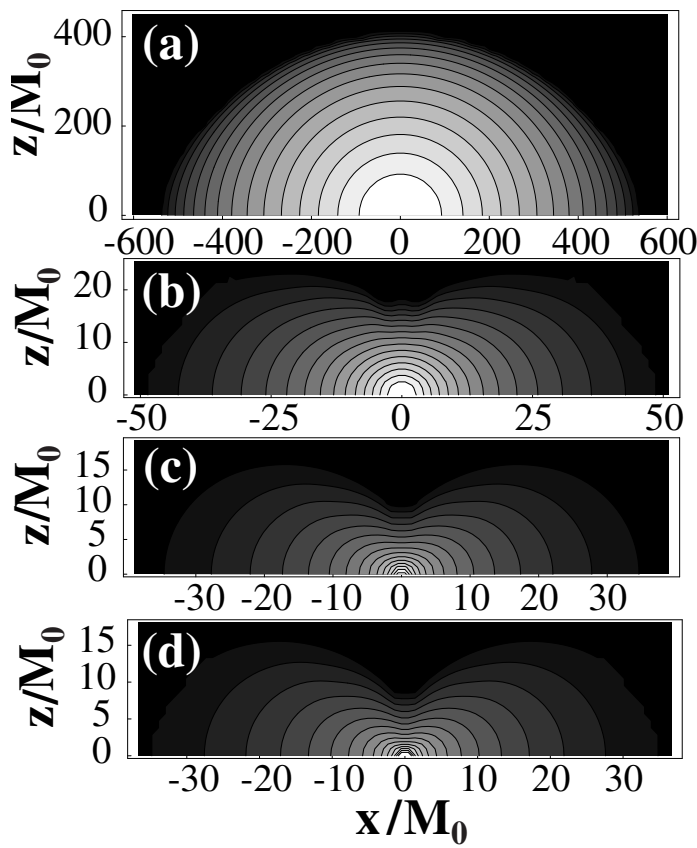

Figure 31: Meridional plane density contours from the SMS collapse simulation of Saijo, Baumgarte, Shapiro, and Shibata [259]. The contour lines denote densities $\rho=\rho_{\mathrm{c}} \times d^{(1-i / 16)}$, where $\rho_{\mathrm{c}}$ is the central density. The frames are plotted at $\left(t / t_{\mathrm{D}}, \rho_{\mathrm{c}}, d\right)=(\mathrm{a})\left(5.0628 \times 10^{-4}, 8.254 \times 10^{-9}, 10^{-7}\right)$, (b) $(2.50259$, $\left.1.225 \times 10^{-4}, 10^{-5}\right)$, (c) $\left(2.05360,8.328 \times 10^{-3}, 5.585 \times 10^{-7}\right)$, (d) $\left(2.50405,3.425 \times 10^{-2}, 1.357 \times 10^{-7}\right)$. Here $t, t_{\mathrm{D}}$, and $M_{0}$ are the time, dynamical time $\left(=\sqrt{R_{\mathrm{e}}^{3} / M}\right.$, where $R_{\mathrm{e}}$ is the initial equatorial radius and $M$ is the mass), and rest mass. (Figure 15 of [259]; used with permission.) 
Saijo et al. also consider the GW emission from the ringdown of the black-hole remnant. For the $l=m=2$ quasi-normal mode of a Kerr black hole with $a / M=0.9$, they estimate the characteristic amplitude of emission to be $h \approx 1.2 \times 10^{-20}\left[\left(\triangle E_{\mathrm{GW}} / M\right) / 10^{-4}\right]^{1 / 2}$ at $f_{\mathrm{GW}} \sim 2 \times 10^{-2} \mathrm{~Hz}$ for an $M=10^{6} M_{\odot}$ source located at a luminosity distance of $50 \mathrm{Gpc}$ (see [174, 307, 285] for details). Here, $\triangle E_{\mathrm{GW}} / M$ is the radiated energy efficiency and may be $\lesssim 7 \times 10^{-4}$ [291]. This GW signal is within LISA's range of sensitivity (see Figure 30). 


\section{Summary}

How likely are we to detect GWs from stellar collapse? And if we detect them, will we be able to use the observation to constrain our understanding of their source? The former question has been at the head of almost every investigation of GWs from gravitational collapse. But with the almost certain detection of neutron-star mergers with advanced $\mathrm{LIGO}^{12}$, the question of detection is gradually being superseded by the question of what we will learn from the detection. To answer either question, a key first step is to determine how nearby the stellar collapse must be to be detectable by current or up-and-coming instruments. Improvements on both the LIGO and VIRGO detectors as well as new observatories, such as LCGT, will soon make GW astronomy a reality. For SMSs, low-frequency detectors like LISA will also play a role. For our discussion, we will use LIGO and LISA as our guides, but bear in mind that these other detectors have comparable detection limits and will be crucial for the success of GW astronomy.

\subsection{Detection of collapse GW signals}

Let's review some of the GW sources and mechanisms discussed in this paper. Figure 32 shows the GW signal from matter motions from bounce and convection in normal stellar collapse assuming a source at $10 \mathrm{kpc}$ (within the Milky Way). The strong signals presented here are upper limits, assuming asymmetries and stellar spin rates that are greater than the values that are produced in the current best-estimates from stellar models (current models are better at placing upper limits than quantitative results). The corresponding signal from neutrinos is shown in Figure 33. For asymmetric collapse, the neutrino-induced GW signal may dominate the signal detected by LIGO. But, in general, it is likely that the matter-induced signal will dominate the signal observed by LIGO.

In general, the signal at bounce is strongest for the rotating (and most asymmetric) explosions. The strong bounce signal is possible in AICs, low-mass collapse and normal core-collapse supernovae. The rest of the signals in Figures 32 and 33 are only appropriate for normal core-collapse supernovae. Except in the fastest-rotating cases, the signals from AICs, low-mass collapse and normal supernovae are only observable if they occur within local group galaxies. We are unable to observe stellar collapse in the nearby Virgo cluster. A detection of a stellar collapse in this cluster would argue that either extreme rotation does occur in stars or that bar modes exist in these cores.

If bar modes do develop in the proto neutron star, the GW signal may be orders of magnitude higher amplitude than that produced in the bounce or convective phase. Figure 23 shows the signal for bar-mode sources assuming the source is at $10 \mathrm{Mpc}$ instead of the $10 \mathrm{kpc}$. Dynamical bar modes could be easily detected out to the Virgo cluster. Even secular bar modes should be detected out to Virgo. If such modes are produced in even 1/10th of all stellar collapses, advanced LIGO should detect multiple GW outbursts per year as soon as it reaches its design specifications. Non-detections place limits on the spin rate of stars.

Black hole forming systems have the potential to form stronger GWs. But the fragmentation claimed in many results did not occur in the 3-dimensional models by Rockefeller et al. [250]. Their predicted GW signal was on par with the upper limits to rotational collapse shown in Figure 32. Like normal stellar collapse, black-hole-forming objects are unlikely to be observed as far out as the Virgo cluster.

Although the signal for very massive stars $\left(\sim 300 M_{\odot}\right)$ is expected to be much larger than other black-hole-forming systems, these objects are believed to only form in the early universe (modest metallicities cause these stars to lose most of their mass in winds, in case they behave more like lower-mass black-hole-forming systems). At such distances, these collapses will be difficult to

\footnotetext{
12 Even the more pessimistic/realistic estimates of the rate of neutron-star mergers predict that such a detection will occur within a few years of operation.
} 


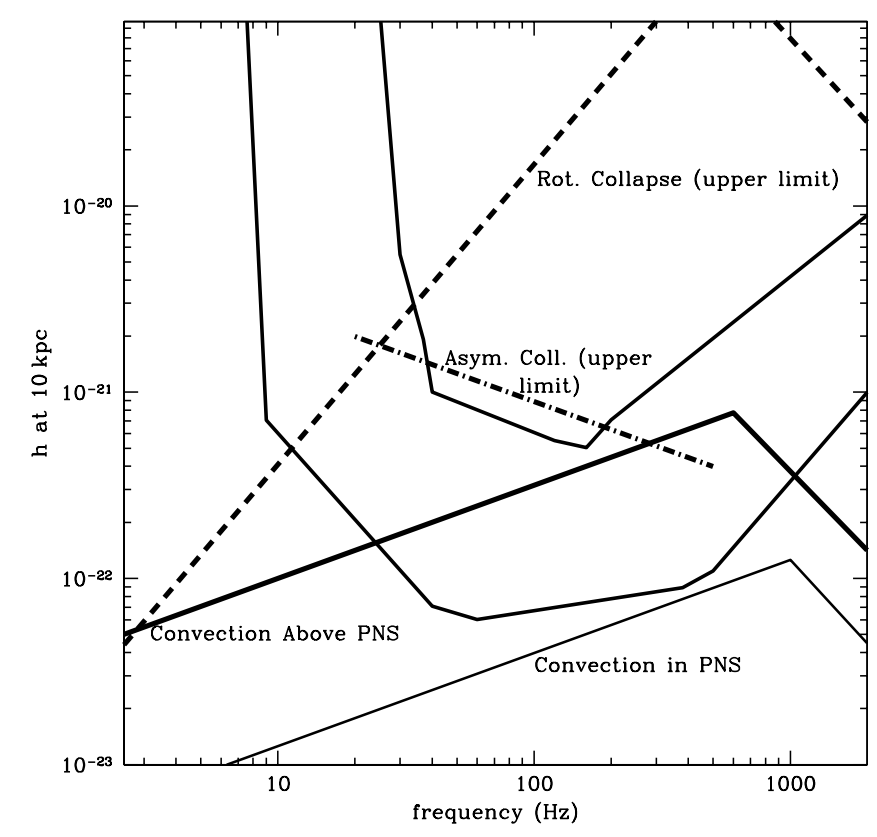

Figure 32: GW signals from matter motions in the bounce and convection phases of stellar collapse. These limits and estimates have not changed from the previous publication of this Living Reviews article [111]. Uncertainties in the estimates still allow variations within an order of magnitude of these results. The LIGO and advanced LIGO sensitivities are included for reference. The limits are not upper limits of a single signal, but upper limits of all possible signals (varying the conditions of the collapse). This figure does not represent a single signal. 


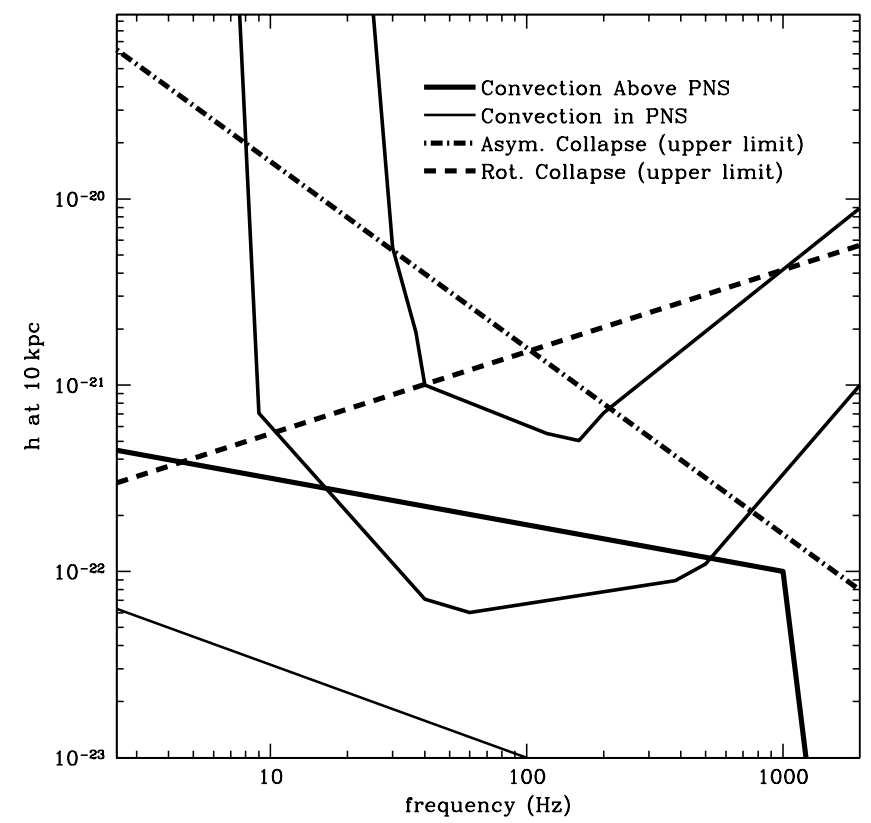

Figure 33: GW signals from asymmetric neutrino emission in the bounce and convection phases of stellar collapse. These limits and estimates have not changed from the previous publication of this Living Reviews article [111]. Uncertainties in the estimates still allow variations within an order of magnitude of these results. The LIGO and advanced LIGO sensitivities are included for reference. The limits are not upper limits of a single signal, but upper limits of all possible signals (varying the conditions of the collapse). This figure does not represent a single signal. 
observe. However, if the rate of these objects is high, it may be that future detectors such as the DECi-hertz Interferometer Gravitational wave Observatory (DECIGO) and the Big Bang Observer (BBO) may be able to detect GW emission from these objects [298].

The possible exception for detectable GW emission from black-hole formation is the formation of SMBHs. SMBHs exist. If they are formed in the collapse of an SMS, a number of observational sources should allow us to observe these objects with LISA (Figure 30).

\subsection{Using GWs to study core collapse}

Beyond the detection of GWs, GW observations of stellar collapse allow us to probe the mechanism behind core-collapse explosions. Along with neutrinos, GWs provide one of the few windows into the heart of the core-collapse supernova explosion mechanism. As we have already discussed, the detection (or lack thereof) of a GW signal can tell us something about the spin rate of the core. For instance, a strong signal from a supernova at the Virgo cluster would require bar-mode instabilities and, hence, a rotation rate in the core that is much faster than we currently believe. Likewise, a strong GRB GW signal would imply fragmentation in the disk that we, as yet, do not observe. This would lead to a major rethinking in the GRB engine.

Scientists also argue that the strength and form of the GW signal in a galactic supernova would allow us to distinguish between asymmetric and rotating collapse and determine the magnitude of rotation (or degree of asymmetry) in these collapse models (e.g., [107, 228, 297]). If we knew the theoretical signal unambiguously, scientists could determine the progenitor rate and degree of differential rotation [297]. Unfortunately, at this time, the range of results discussed in this review is too large for such a study; it points out the potential for GW to address very specific astronomy questions if collapse models reach some agreement on the exact GW signal.

But precision measurements in gravitational collapse have been hampered by the lack of true verification and validation studies of the existing codes. Although the amount of angular momentum in the core is critical to determining the exact wave signal, very few solid tests of angular momentum conservation (and artificial angular-momentum transport) have been made on most codes. This issue is even more important for three-dimensional calculations. Until this source of error can be quantified and minimized, the errors in the GW signal will remain large, stagnating this field and limiting what we can learn from an observed signal.

The detection of GWs from weak or dim supernovae will provide clues to these rarely-observed classes of supernovae. Dim supernovae can be produced both by the collapse of low-mass stars (electron-capture supernovae) or the collapse of massive stars that have considerable fallback. Models argue that these explosions are difficult to observe and such supernovae may occur nearby without detection in the optical. GWs may provide the only direct means to study these supernovae. Similarly, different GRB models predict very different GW signals and the GW detection of a GRB will place strong constraints on the current engine models.

Finally, detectors such as LIGO will build up a sample of merging systems consisting of neutron stars or black holes. From these detections, we can derive strong observational constraints on the birth mass distributions of both neutron stars and stellar-massed black holes. This set of observations will indirectly constrain the explosion mechanisms for core collapse.

Current studies have begun to probe the tip of the iceberg of what we will be able to study in core collapse with GWs and we expect the number of papers probing this topic to increase dramatically in the next few years. 


\section{Acknowledgements}

It is a pleasure to thank John Blondin, Adam Burrows, Thierry Foglizzo, Scott Hughes, Kei Kotake, Paolo Mazzali, Ewald Müller, Ken'ichi Nomoto, Shangli Ou, Gabriel Rockefeller, and Stuart Shapiro for helpful conversations and/or permission to reprint figures/movies from their published works. This work was carried out in part under the auspices of the National Nuclear Security Administration of the Department of Energy (DOE) at Los Alamos National Laboratory and supported by a LANL/LDRD program. 


\section{References}

[1] Abel, T., Bryan, G.L. and Norman, M.L., "The Formation and Fragmentation of Primordial Molecular Clouds", Astrophys. J., 540, 39-44, (2000). [DOI], [ADS], [arXiv:astro-ph/0002135]. (Cited on page 29.)

[2] Akiyama, S. and Wheeler, J.C., "Magnetic Fields in Supernovae", in Fryer, C.L., ed., Stellar Collapse, Proceedings of 'Core Collapse of Massive Stars', 200th AAS meeting, Albuquerque, NM, June 2002, Astrophysics and Space Science Library, 302, pp. 259-275, (Kluwer Academic Publishers, Dordrecht; Boston, 2004). [ADS], [astro-ph/0211458], [Google Books]. (Cited on pages 18,27 , and 37 .)

[3] Akiyama, S., Wheeler, J.C., Meier, D.L. and Lichtenstadt, I., "The Magnetorotational Instability in Core-Collapse Supernova Explosions", Astrophys. J., 584, 954-970, (2003). [DOI], [ADS], [arXiv:astro-ph/0208128]. (Cited on pages 18 and 27.)

[4] Andersson, N., "A New Class of Unstable Modes of Rotating Relativistic Stars", Astrophys. J., 502, 708-713, (1998). [DOI], [ADS], [arXiv:gr-qc/9706075]. (Cited on pages 9 and 57.)

[5] Andersson, N., "Gravitational waves from instabilities in relativistic stars", Class. Quantum Grav., 20, R105-R144, (2003). [DOI], [ADS], [arXiv:astro-ph/0211057]. (Cited on pages 9, 48, and 57.)

[6] Ardeljan, N.V., Bisnovatyi-Kogan, G.S. and Moiseenko, S.G., "Magnetorotational supernovae", Mon. Not. R. Astron. Soc., 359, 333-344, (2005). [DOI], [ADS]. (Cited on pages 18 and 27.)

[7] Arnaud, N. et al., "Detection of a close supernova gravitational wave burst in a network of interferometers, neutrino and optical detectors", Astropart. Phys., 21, 201-221, (2004). [DOI], [ADS]. (Cited on page 5.)

[8] Arras, P., Flanagan, É.É., Morsink, S.M., Schenk, A.K., Teukolsky, S.A. and Wasserman, I., "Saturation of the $r$-mode instability", Astrophys. J., 591, 1129-1151, (2002). [DOI], [ADS], [arXiv:astro-ph/0202345]. (Cited on page 57.)

[9] Baiotti, L., Hawke, I. and Rezzolla, L., "On the gravitational radiation from the collapse of neutron stars to rotating black holes", Class. Quantum Grav., 24, S187-S206, (2007). [DOI], [ADS]. (Cited on page 60.)

[10] Bardeen, J.M. and Piran, T., "General relativistic axisymmetric rotating systems: Coordinates and equations", Phys. Rep., 96, 205-250, (1983). [DOI], [ADS]. (Cited on page 32.)

[11] Baron, E., Cooperstein, J., Kahana, S. and Nomoto, K., "Collapsing white dwarfs", Astrophys. J., 320, 304-307, (1987). [DOI], [ADS]. (Cited on pages 25 and 26.)

[12] Baumgarte, T.W. and Shapiro, S.L., "Evolution of Rotating Supermassive Stars to the Onset of Collapse", Astrophys. J., 526, 941-952, (1999). [DOI], [ADS], [arXiv:astro-ph/9909237]. (Cited on pages 30,60, and 61.)

[13] Bazan, G. and Arnett, D., "Convection, Nucleosynthesis, and Core Collapse", Astrophys. J. Lett., 433, L41-L43, (1994). [DOI], [ADS]. (Cited on page 41.)

[14] Begelman, M.C. and Rees, M.J., "The fate of dense stellar systems", Mon. Not. R. Astron. Soc., 185, 847-859, (1978). [ADS]. (Cited on page 29.) 
[15] Begelman, M.C., Volunteri, M. and Rees, M.J., "Formation of supermassive black holes by direct collapse in pre-galactic haloes", Mon. Not. R. Astron. Soc., 370, 289-298, (2006). [ADS]. (Cited on page 30.)

[16] Benz, W., Bowers, R.L., Cameron, A.G.W. and Press, W.H., "Dynamic Mass Exchange in Doubly Degenerate Binaries. I. 0.9 and $1.2 M_{\odot}$ Stars", Astrophys. J., 348, 647-667, (1990). [DOI], [ADS]. (Cited on page 25.)

[17] Berti, E. and Cardoso, V., "Quasinormal ringing of Kerr black holes: The excitation factors", Phys. Rev. D, 74, 104020, (2006). [DOI], [ADS]. (Cited on page 60.)

[18] Berti, E., Cardoso, V. and Will, C.M., "Gravitational-wave spectroscopy of massive black holes with the space interferometer LISA", Phys. Rev. D, 73, 064030, (2006). [DOI], [ADS], [arXiv:gr-qc/0512160]. (Cited on page 60.)

[19] Bethe, H.A. and Wilson, J.R., "Revival of a stalled supernova shock by neutrino heating", Astrophys. J., 295, 14-23, (1985). [DOI], [ADS]. (Cited on page 13.)

[20] Blondin, J.M. and Mezzacappa, A., "The Spherical Accretion Shock Instability in the Linear Regime", Astrophys. J., 642, 401-409, (2006). [DOI], [ADS]. (Cited on page 18.)

[21] Blondin, J.M., Mezzacappa, A. and DeMarino, C., "Stability of Standing Accretion Shocks, with an Eye toward Core-Collapse Supernovae", Astrophys. J., 584, 971-980, (2003). [DOI], [ADS]. (Cited on pages 18 and 22.)

[22] Bodenheimer, P. and Ostriker, J.P., "Rapidly Rotating Stars. VIII. Zero-viscosity Polytropic Sequences", Astrophys. J., 180, 159-169, (1973). [DOI], [ADS]. (Cited on page 30.)

[23] Bonazzola, S. and Marck, J.-A., "Efficiency of gravitational radiation from axisymmetric and 3D stellar collapse. I. Polytropic case", Astron. Astrophys., 267, 623-633, (1993). [ADS]. (Cited on page 51.)

[24] Bondarescu, R., Teukolsky, S.A. and Wasserman, I., "Spinning down newborn neutron stars: Nonlinear development of the $r$-mode instability", Phys. Rev. D, 79, 104003, (2009). [DOI], [ADS], [arXiv:0809.3448]. (Cited on page 57.)

[25] Braginsky, V.B. and Thorne, K.S., "Gravitational-wave bursts with memory and experimental prospects", Nature, 327, 123-125, (1987). [DOI]. (Cited on page 12.)

[26] Brink, J., Teukolsky, S.A. and Wasserman, I., "Nonlinear coupling network to simulate the development of the $\mathrm{r}$ mode instability in neutron stars. I. Construction", Phys. Rev. D, 70, 124017, (2004). [DOI], [ADS], [arXiv:gr-qc/0409048]. (Cited on page 57.)

[27] Brink, J., Teukolsky, S.A. and Wasserman, I., "Nonlinear couplings of R-modes: Energy transfer and saturation amplitudes at realistic timescales", Phys. Rev. D, 70, 121501, (2004). [DOI], [ADS], [arXiv:gr-qc/0406085]. (Cited on page 57.)

[28] Brink, J., Teukolsky, S.A. and Wasserman, I., "Nonlinear coupling network to simulate the development of the $\mathrm{r}$ mode instability in neutron stars. II. Dynamics", Phys. Rev. D, 71, 064029, (2005). [DOI], [ADS]. (Cited on page 57.)

[29] Bromm, V., Coppi, P.S. and Larson, R.B., "Forming the First Stars in the Universe: The Fragmentation of Primordial Gas", Astrophys. J., 527, L5-L8, (1999). [DOI], [ADS], [arXiv:astro-ph/9910224]. (Cited on page 29.) 
[30] Brown, J.D., "Gravitational waves from the dynamical bar instability in a rapidly rotating star", Phys. Rev. D, 62, 084024, 1-11, (2000). [DOI], [ADS], [arXiv:gr-qc/0004002]. (Cited on pages 48 and 51.)

[31] Brown, J.D., "Rotational instabilities in post-collapse stellar cores", in Centrella, J.M., ed., Astrophysical Sources for Ground-Based Gravitational Wave Detectors, Philadelphia, Pennsylvania, 30 October -1 November 2000, AIP Conference Proceedings, 575, pp. 234-245, (American Institute of Physics, Melville, 2001). [gr-qc/0012084]. (Cited on page 52.)

[32] Bruenn, S.W., "Numerical simulations of core collapse supernovae", in Guidry, M.W. and Strayer, M.R., eds., Nuclear Physics in the Universe, Proceedings of the First Symposium on Nuclear Physics in the Universe held in Oak Ridge, Tennessee, USA, 24-26 September 1992, pp. 31-50, (Institute of Physics, Bristol; Philadelphia, 1993). [ADS]. (Cited on page 45.)

[33] Bruenn, S.W., De Nisco, K.R. and Mezzacappa, A., "General Relativistic Effects in the Core Collapse Supernova Mechanism", Astrophys. J., 560, 326-338, (2001). [DOI], [ADS], [arXiv:astro-ph/0101400]. (Cited on page 36.)

[34] Buras, R., Janka, H.-T., Rampp, M. and Kifonidis, K., "Two-dimensional hydrodynamic core-collapse supernova simulations with spectral neutrino transport. II. Models for different progenitor stars", Astron. Astrophys., 457, 281-308, (2006). [ADS]. (Cited on pages 22 and 36.)

[35] Burrows, A., Dessart, L., Livne, E., Ott, C.D. and Murphy, J., "Simulations of Magnetically Driven Supernova and Hypernova Explosions in the Context of Rapid Rotation", Astrophys. J., 664, 416-434, (2007). [DOI], [ADS]. (Cited on pages 18 and 27.)

[36] Burrows, A. and Goshy, J., "A Theory of Supernova Explosions", Astrophys. J. Lett., 416, L75-L78, (1993). [ADS]. (Cited on page 13.)

[37] Burrows, A. and Hayes, J., "Pulsar recoil and gravitational radiation due to asymmetrical stellar collapse and explosion", Phys. Rev. Lett., 76, 352-355, (1996). [DOI], [ADS], [arXiv:astro-ph/9511106]. (Cited on pages 41 and 42.)

[38] Burrows, A., Livne, E., Dessart, L., Ott, C.D. and Murphy, J., "An acoustic mechanism for core-collapse supernova explosions", New Astron. Rev., 50, 487-491, (2006). [DOI], [ADS]. (Cited on page 18.)

[39] Burrows, A., Livne, E., Dessart, L., Ott, C.D. and Murphy, J., "Features of the Acoustic Mechanism of Core-Collapse Supernova Explosions", Astrophys. J., 655, 416-433, (2006). [DOI], [ADS]. (Cited on page 18.)

[40] Burrows, A., Livne, E., Dessart, L., Ott, C.D. and Murphy, J., "A New Mechanism for Core-Collapse Supernova Explosions", Astrophys. J., 640, 878-890, (2006). [DOI], [ADS], [arXiv:astro-ph/0510687]. (Cited on pages 18, 22, 45, and 47.)

[41] Calder, A.C. et al., "On Validating an Astrophysical Simulation Code", Astrophys. J. Suppl. Ser., 143, 201-229, (2002). [DOI], [ADS], [arXiv:astro-ph/0206251]. (Cited on page 19.)

[42] Cantiello, M., Yoon, S.-C., Langer, N. and Livio, M., "Binary star progenitors of long gammaray bursts", Astron. Astrophys., 465, L29-L33, (2007). [DOI], [ADS]. (Cited on pages 9 and 49.)

[43] Cappellaro, E., Evans, R. and Turatto, M., "A new determination of supernova rates and a comparison with indicators for galactic star formation", Astron. Astrophys., 351, 459-466, (1999). [ADS]. (Cited on page 19.) 
[44] Cappellaro, E. et al., "Death rate of massive stars at redshift $~ 0.3 "$, Astron. Astrophys., 430, 83-93, (2005). [DOI], [ADS]. (Cited on page 20.)

[45] Centrella, J.M. and McMillan, S.L.W., "Gravitational Radiation from Nonaxisymmetric Collisions of Neutron Stars", Astrophys. J., 416, 719-732, (1993). [DOI], [ADS]. (Cited on page 10.)

[46] Centrella, J.M., New, K.C.B., Lowe, L.L. and Brown, J.D., "Dynamical rotational instability at low T/W", Astrophys. J. Lett., 550, L193-L196, (2001). [DOI], [ADS]. (Cited on pages 49, $50,51,52$, and 53.)

[47] Cerdá-Durán, P., Font, J.A., Antón, L. and Müller, E., "A new general relativistic magnetohydrodynamics code for dynamical spacetimes", Astron. Astrophys., 492, 937-953, (2008). [DOI], [ADS]. (Cited on page 36.)

[48] Cerdá-Durán, P., Quilis, V. and Font, J.A., "AMR simulations of the low $T /|W|$ bar-mode instability of neutron stars", Comput. Phys. Commun., 177, 288-297, (2007). [DOI], [ADS]. (Cited on page 36.)

[49] Chandrasekhar, S., "Dynamical Instability of Gaseous Masses Approaching the Schwarzschild Limit in General Relativity", Phys. Rev. Lett., 12, 114-116, (1964). [DOI]. (Cited on page 30.)

[50] Chandrasekhar, S., "The Dynamical Instability of Gaseous Masses Approaching the Schwarzschild Limit in General Relativity", Astrophys. J., 140, 417-433, (1964). [DOI], [ADS]. (Cited on page 30.)

[51] Chandrasekhar, S., An Introduction to the Study of Stellar Structure, (Dover, New York, 1967). (Cited on page 50.)

[52] Chandrasekhar, S., "The Effect of Gravitational Radiation on the Secular Stability of the Maclaurin Spheroid", Astrophys. J., 161, 561-569, (1970). [DOI], [ADS]. (Cited on page 48.)

[53] Chatterjee, D. and Bandyopadhyay, D., "Role of antikaon condensation in r-mode instability", in Santra, A.B., ed., Physics and Astrophysics of Hadrons and Hadronic Matter, Shantiniketan, India, 6-10 Nov 2006, p. 237, (Narosa Publishing House, New Delhi, 2008). [ADS], [arXiv:0712.4347]. (Cited on page 57.)

[54] Chevalier, R.A., "Neutron star accretion in a supernova", Astrophys. J., 346, 847-859, (1989). [DOI], [ADS]. (Cited on page 27.)

[55] Colgate, S.A., "Neutron-Star Formation, Thermonuclear Supernovae, and Heavy-Element Reimplosion", Astrophys. J., 163, 221-230, (1971). [DOI], [ADS]. (Cited on page 27.)

[56] Colgate, S.A. and White, R.H., "The Hydrodynamic Behavior of Supernovae Explosions", Astrophys. J., 143, 626-681, (1966). [DOI], [ADS]. (Cited on page 13.)

[57] Cook, G.B., Shapiro, S.L. and Teukolsky, S.A., "Testing a simplified version of Einstein's equations for numerical relativity", Phys. Rev. D, 53, 5533-5540, (1996). [DOI], [ADS], [arXiv:astro-ph/9512009]. (Cited on page 37.)

[58] Couch, R.G. and Arnett, W.D., "On the Thermal Properties of the Convective URCA Process", Astrophys. J., 194, 537-539, (1974). [DOI], [ADS]. (Cited on page 25.)

[59] Dahlen, T. et al., "High-Redshift Supernova Rates", Astrophys. J., 613, 189-199, (2004). [DOI], [ADS]. (Cited on page 20.) 
[60] Dessart, L., Burrows, A., Livne, E. and Ott, C.D., "Multidimensional Radiation/Hydrodynamic Simulations of Proto-Neutron Star Convection", Astrophys. J., 645, 534-550, (2006). [DOI], [ADS]. (Cited on page 22.)

[61] Dessart, L., Burrows, A., Livne, E. and Ott, C.D., "Magnetically Driven Explosions of Rapidly Rotating White Dwarfs Following Accretion-Induced Collapse", Astrophys. J., 669, 585-599, (2007). [DOI], [ADS]. (Cited on pages 9, 18, 24, 25, 26, and 49.)

[62] Dessart, L., Burrows, A., Livne, E. and Ott, C.D., "The Proto-Neutron Star Phase of the Collapsar Model and the Route to Long-Soft Gamma-Ray Bursts and Hypernovae", Astrophys. J., 673, L43-L46, (2008). [DOI], [ADS]. (Cited on page 18.)

[63] Dessart, L., Burrows, A., Ott, C.D., Livne, E., Yoon, S.-C. and Langer, N., "Multidimensional Simulations of the Accretion-induced Collapse of White Dwarfs to Neutron Stars", Astrophys. J., 644, 1063-1084, (2006). [DOI], [ADS]. (Cited on pages 9, 25, 26, and 49.)

[64] Detweiler, S. and Lindblom, L., "On the Evolution of the Homogeneous Ellipsoidal Figures. II. Gravitational Collapse and Gravitational Radiation", Astrophys. J., 250, 739-749, (1981). [DOI], [ADS]. (Cited on pages 6 and 32.)

[65] Dimmelmeier, H., Font, J.A. and Müller, E., "Gravitational Waves from Relativistic Rotational Core Collapse", Astrophys. J. Lett., 560, L163-L166, (2001). [DOI], [ADS], [arXiv:astroph/0103088]. (Cited on page 37.)

[66] Dimmelmeier, H., Font, J.A. and Müller, E., "Relativistic simulations of rotational core collapse I. Methods, initial models, and code tests", Astron. Astrophys., 388, 917-935, (2002). [DOI], [ADS], [arXiv:astro-ph/0204288]. (Cited on page 37.)

[67] Dimmelmeier, H., Font, J.A. and Müller, E., "Relativistic simulations of rotational core collapse II. Collapse dynamics and gravitational radiation", Astron. Astrophys., 393, 523542, (2002). [DOI], [ADS], [arXiv:astro-ph/0204289]. (Cited on pages 6, 37, 38, 39, and 53.)

[68] Dimmelmeier, H., Ott, C.D., Janka, H.T., Marek, A. and Müller, E., "Generic GravitationalWave Signals from the Collapse of Rotating Stellar Cores", Phys. Rev. D, 98, 251101, (2007). [DOI], [ADS], [arXiv:astro-ph/0702305]. (Cited on pages 6, 36, and 37.)

[69] Dimmelmeier, H., Ott, C.D., Marek, A. and Janka, H.-T., "Gravitational wave burst signal from core collapse of rotating stars", Phys. Rev. D, 78, 064056, (2008). [DOI], [ADS]. (Cited on pages $9,19,36,37$, and 49 .)

[70] Dimonte, G. et al., "A comparative study of turbulent Rayleigh-Taylor instability using high-resolution three-dimensional numerical simulations: The Alpha-Group collaboration", Phys. Fluids, 16, 1668-1693, (2002). [DOI], [ADS]. (Cited on page 19.)

[71] Drago, A., Pagliara, G. and Parenti, I., "A Compact Star Rotating at $1122 \mathrm{~Hz}$ and the $r$-Mode Instability", Astrophys. J. Lett., 678, L117-L120, (2008). [DOI], [ADS], [arXiv:0704.1510]. (Cited on page 57.)

[72] D'Souza, M.C.R., Motl, P.M., Tohline, J.E. and Frank, J., "Numerical Simulatons of the Onset and Stability of Dynamical Mass Transfer in Binaries", Astrophys. J., 643, 381-401, (2006). [DOI], [arXiv:astro-ph/0512137]. (Cited on page 25.)

[73] Duez, M.D., Shapiro, S.L. and Yo, H.-J., "Relativistic hydrodynamic evolutions with black hole excision", Phys. Rev. D, 69, 104016, 1-16, (2004). [DOI], [ADS]. (Cited on pages 58 and 60.) 
[74] Durisen, R.H. and Tohline, J.E., "Fission of rapidly rotating fluid systems", in Black, D. and Matthews, M., eds., Protostars and Planets II, pp. 534-575, (University of Arizona Press, Tucson, 1985). [ADS]. (Cited on page 48.)

[75] Eisenstein, D.J. and Loeb, A., "Origin of Quasar Progenitors From The Collapse of Low-spin Cosmological Perturbations", Astrophys. J., 443, 11-17, (1995). [DOI], [ADS], [arXiv:astroph/9401016]. (Cited on page 29.)

[76] Epstein, R., "The generation of gravitational radiation by escaping supernova neutrinos", Astrophys. J., 223, 1037-1045, (1976). [DOI], [ADS]. (Cited on pages 11 and 54.)

[77] Epstein, R., The post-Newtonian theory of the generation of gravitational radiation and its application to stellar collapse, Ph.D. Thesis, (Stanford University, Stanford, 1976). [ADS]. (Cited on page 32.)

[78] Epstein, R. and Wagoner, R.V., "Post-Newtonian Generation of Gravitational Waves", Astrophys. J., 197, 717-723, (1975). [DOI], [ADS]. (Cited on page 32.)

[79] Eriguchi, Y. and Müller, E., "Equilibrium models of differentially rotating polytropes and the collapse of rotating stellar cores", Astron. Astrophys., 147, 161-168, (1985). [ADS]. (Cited on page 33.)

[80] Favata, M., "Post-Newtonian corrections to the gravitational-wave memory for quasicircular, inspiralling compact binaries", Phys. Rev. D, 80, 024002, (2009). [DOI], [ADS]. (Cited on page 12.)

[81] Fernández, R. and Thompson, C., "Dynamics of a Spherical Accretion Shock with Neutrino Heating and Alpha-Particle Recombination", Astrophys. J., 703, 1464-1485, (2009). [DOI], [ADS]. (Cited on page 18.)

[82] Fernández, R. and Thompson, C., "Stability of a Spherical Accretion Shock with Nuclear Dissociation", Astrophys. J., 697, 1827-1841, (2009). [DOI], [ADS]. (Cited on page 18.)

[83] Ferrarese, L. and Merritt, D., "A fundamental relation between supermassive black holes and their host galaxies", Astrophys. J. Lett., 539, L9-L12, (2000). [DOI], [ADS]. (Cited on page 29.)

[84] Ferrari, V. and Galtieri, L., "Quasi-normal modes and gravitational wave astronomy", Gen. Relativ. Gravit., 40, 945-970, (2008). [DOI], [ADS]. (Cited on page 60.)

[85] Ferrari, V., Miniutti, G. and Pons, J.A., "Gravitational waves from newly born, hot neutron stars", Mon. Not. R. Astron. Soc., 342, 629-638, (2003). [DOI], [ADS]. (Cited on pages 22, 23,45 , and 53.)

[86] Finn, L.S., "Supernovae, Gravitational Radiation, and the Quadrupole Formula", in Evans, C.R., Finn, L.S. and Hobill, D.W., eds., Frontiers in Numerical Relativity, International workshop devoted to research in numerical relativity, held in Urbana-Champaign in May 1988, pp. 126-145, (Cambridge University Press, Cambridge; New York, 1989). [Google Books]. (Cited on page 33.)

[87] Finn, L.S., "Detectability of gravitational radiation from stellar-core collapse", in Buchler, J.R., Detweiler, S. and Ipser, J.R., eds., Nonlinear problems in relativity and cosmology, 6th Florida Workshop in Nonlinear Astronomy, held on October 2-4, 1990 in Gainesville, Florida, Ann. N.Y. Acad. Sci., 631, pp. 156-172, (New York Academy of Sciences, New York, 1991). [DOI]. (Cited on page 32.) 
[88] Finn, L.S., "Gravitional Radiation Sources and Signatures", in Dixon, L.J., ed., Gravity: From the Hubble Length to the Planck Length, Proceedings of the 26th SLAC Summer Institute on Particle Physics (SSI 98), Stanford, USA, 3-14 August 1998, SLAC-R, 538, (SLAC, Springfield, 2001). URL (accessed 30 March 1999):

http://www.slac.stanford.edu/pubs/confproc/ssi98/ssi98-007.html. (Cited on page 5.)

[89] Finn, L.S. and Evans, C.R., "Determining Gravitational Radiation from Newtonian SelfGravitating Systems", Astrophys. J., 351, 588-600, (1990). [DOI], [ADS]. (Cited on pages 6 and 33.)

[90] Fischer, T., Whitehouse, S.C., Mezzacappa, A., Thielemann, F.-K. and Liebendörfer, M., "The neutrino signal from protoneutron star accretion and black hole formation", Astron. Astrophys., 499, 1-15, (2009). [DOI], [ADS]. (Cited on page 27.)

[91] Fisker, J.L., Balsara, D.S. and Burger, T., "The accretion and spreading of matter on white dwarfs", New Astron. Rev., 50, 509-515, (2006). [DOI], [ADS]. (Cited on page 25.)

[92] Foglizzo, T., "A Simple Toy Model of the Advective-Acoustic Instability. I. Perturbative Approach", Astrophys. J., 694, 820-832, (2009). [DOI], [ADS]. (Cited on page 18.)

[93] Foglizzo, T., Galletti, P., Scheck, L. and Janka, H.-T., "Instability of a Stalled Accretion Shock: Evidence for the Advective-Acoustic Cycle", Astrophys. J., 654, 1006-1021, (2007). [DOI], [ADS]. (Cited on page 18.)

[94] Foglizzo, T., Scheck, L. and Janka, H.-T., "Neutrino-driven Convection versus Advection in Core-Collapse Supernovae", Astrophys. J., 652, 1436-1450, (2006). [DOI], [ADS], [arXiv:astroph/0507636]. (Cited on page 18.)

[95] Folkner, W.M., ed., Laser Interferometer Space Antenna: Second International LISA Symposium on the Detection and Observation of Gravitational Waves in Space, Pasadena 1998, AIP Conference Proceedings, 456, (Springer, New York, 1993). (Cited on page 60.)

[96] Friedman, J.L. and Morsink, S.M., "Axial Instability of Rotating Relativistic Stars", Astrophys. J., 502, 714-720, (1998). [DOI], [ADS], [arXiv:gr-qc/9706073]. (Cited on pages 9 and 57.)

[97] Fryer, C.L., "Mass Limits For Black Hole Formation", Astrophys. J., 522, 413-418, (1999). [DOI], [ADS]. (Cited on pages 13, 14, 27, and 31.)

[98] Fryer, C.L., "Neutron Star Kicks from Asymmetric Collapse", Astrophys. J. Lett., 601, L175-L178, (2004). [DOI], [ADS]. (Cited on pages 36 and 41.)

[99] Fryer, C.L., "Fallback in stellar collapse", New Astron. Rev., 50, 492-495, (2006). [DOI], [ADS]. (Cited on pages 15, 17, and 22.)

[100] Fryer, C.L., "Neutrinos from Fallback onto Newly Formed Neutron Stars", Astrophys. J., 699, 409-420, (2009). [DOI], [ADS]. (Cited on pages 18 and 27.)

[101] Fryer, C.L., Benz, W. and Herant, M., "The Dynamics and Outcomes of Rapid Infall onto Neutron Stars", Astrophys. J., 460, 801-826, (1996). [DOI], [ADS]. (Cited on pages 15 and 18.)

[102] Fryer, C.L., Benz, W., Herant, M. and Colgate, S.A., "What can the accretion-induced collapse of white dwarfs really explain?", Astrophys. J., 516, 892-899, (1999). [DOI], [ADS]. (Cited on pages 24, 25, 26, and 50.) 
[103] Fryer, C.L. and Heger, A., "Core-Collapse Simulations of Rotating Stars", Astrophys. J., 541, 1033-1050, (2000). [DOI], [ADS], [arXiv:astro-ph/9907433]. (Cited on pages 36, 37, and 49.)

[104] Fryer, C.L. and Heger, A., "Binary Merger Progenitors for Gamma-Ray Bursts and Hypernovae", Astrophys. J., 623, 302-313, (2005). [DOI], [ADS]. (Cited on pages 9, 28, and 49.)

[105] Fryer, C.L., Herwig, F., Hungerford, A. and Timmes, F.X., "Supernova Fallback: A Possible Site for the $r$-Process", Astrophys. J. Lett., 646, L131-L134, (2006). [DOI], [ADS], [arXiv:astro$\mathrm{ph} / 0606450]$. (Cited on page 27.)

[106] Fryer, C.L., Holz, D.E. and Hughes, S.A., "Gravitational Wave Emission from Core Collapse of Massive Stars", Astrophys. J., 565, 430-446, (2002). [DOI], [ADS]. (Cited on pages 6, 7, $8,9,36,37,48,57,58$, and 60.$)$

[107] Fryer, C.L., Holz, D.E. and Hughes, S.A., "Gravitational Waves from Stellar Collapse: Correlations to Explosion Asymmetries", Astrophys. J., 609, 288-300, (2004). [DOI], [ADS]. (Cited on pages $5,11,36,42,43,54,56$, and 67 .)

[108] Fryer, C.L., Holz, D.E., Hughes, S.A. and Warren, M.S., "Stellar collapse and gravitational waves", in Fryer, C.L., ed., Stellar Collapse, Proceedings of 'Core Collapse of Massive Stars', 200th AAS meeting, Albuquerque, NM, June 2002, Astrophysics and Space Science Library, 302, pp. 373-401, (Kluwer Academic Publishers, Dordrecht; Boston, 2004). [ADS], [astroph/0211609]. (Cited on pages 5, 6, 36, 48, and 58.)

[109] Fryer, C.L. and Kalogera, V., "Theoretical Black Hole Mass Distributions", Astrophys. J., 554, 548-560, (2001). [DOI], [ADS]. (Cited on pages 13, 15, and 29.)

[110] Fryer, C.L. and Kusenko, A., "Effects of Neutrino-driven Kicks on the Supernova Explosion Mechanism", Astrophys. J., 163, 335-343, (2006). [DOI], [ADS], [arXiv:astro-ph/0512033]. (Cited on page 36.)

[111] Fryer, C.L. and New, K.C.B, "Gravitational Waves from Gravitational Collapse", Living Rev. Relativity, 6, lrr-2003-2, (2003). URL (accessed 18 May 2009): http://www.livingreviews.org/lrr-2003-2. (Cited on pages 65 and 66.)

[112] Fryer, C.L. and Warren, M.S., "Modeling Core-Collapse Supernovae in Three Dimensions", Astrophys. J. Lett., 574, L65-L68, (2002). [DOI], [ADS], [arXiv:astro-ph/0206017]. (Cited on pages 22,36 , and 46.$)$

[113] Fryer, C.L. and Warren, M.S., "The Collapse of Rotating Massive Stars in Three Dimensions", Astrophys. J., 601, 391-404, (2004). [DOI], [ADS]. (Cited on pages 8, 9, 19, 22, 36, and 49.)

[114] Fryer, C.L., Woosley, S.E. and Hartmann, D.H., "Formation Rates of Black Hole Accretion Disk Gamma-Ray Bursts", Astrophys. J., 526, 152-177, (1999). [DOI], [ADS]. (Cited on pages 9,27 , and 49 .)

[115] Fryer, C.L., Woosley, S.E. and Heger, A., "Pair-Instability Supernovae, Gravity Waves, and Gamma-Ray Transients", Astrophys. J., 550, 372-382, (2001). [DOI], [ADS], [arXiv:astroph/0007176]. (Cited on pages 13, 15, 29, 49, and 50.)

[116] Fryer, C.L. and Young, P.A., "Late-Time Convection in the Collapse of a $23 M_{\odot}$ Star", Astrophys. J., 659, 1438-1448, (2007). [DOI], [ADS]. (Cited on pages 18, 19, 21, 22, and 45.)

[117] Fryer, C.L. et al., "The Supernova Gamma-Ray Burst Connection", Publ. Astron. Soc. Pac., 119, 1211-1232, (2007). [DOI], [ADS]. (Cited on page 18.) 
[118] Fryer, C.L. et al., "Spectra and Light Curves of Failed Supernovae", Astrophys. J., 707, 193-207, (2009). [DOI], [ADS]. (Cited on pages 9 and 49.)

[119] Fu, W. and Lai, D., "Low $=T /|W|$ instabilities in differentially rotating proto-neutron stars with magnetic fields", Mon. Not. R. Astron. Soc., submitted, (2010). [arXiv:1011.4887]. (Cited on page 51.)

[120] Fuller, G.M., Kusenko, A., Mociouiu, I. and Pascoli, S., "Pulsar kicks from a dark-matter sterile neutrino", Phys. Rev. D, 68, 103002, (2003). [DOI], [ADS]. (Cited on pages 22 and 23.)

[121] Gentle, A.P. and Miller, W.A., "A fully (3+1)-dimensional Regge calculus model of the Kasner cosmology", Class. Quantum Grav., 15, 389-405, (1965). [DOI], [ADS]. (Cited on page 12.)

[122] "GEO600: The German-British Gravitational Wave Detector", project homepage, MPI for Gravitational Physics (Albert Einstein Institute). URL (accessed 19 January 2010): http://www.geo600.org/. (Cited on page 5.)

[123] Goldreich, P. and Lynden-Bell, D., "I. Gravitational stability of uniformly rotating disks", Mon. Not. R. Astron. Soc., 130, 97-124, (1965). [ADS]. (Cited on page 8.)

[124] "Gravitational Radiation from General Relativistic Rotational Core Collapse", project homepage, Max Planck Institute for Astrophysics, (2002). URL (accessed 7 January 2009): http://www.mpa-garching.mpg.de/rel_hydro/axi_core_collapse/index.shtml. (Cited on page 37.)

[125] Gressman, P., Lin, L.-M., Suen, W.-M., Stergioulas, N. and Friedman, J.L., "Nonlinear $r$ modes in neutron stars: Instability of an unstable mode", Phys. Rev. D, 66, 041303, 1-5, (2002). [DOI], [ADS], [arXiv:gr-qc/0301014]. (Cited on page 57.)

[126] Guerrero, J., García-Berro, E. and Isern, J., "Smoothed Particle Hydrodynamics simulations of merging white dwarfs", Astron. Astrophys., 413, 257-272, (2004). [DOI], [ADS]. (Cited on page 25.)

[127] Gutiérrez, J., Canal, R. and García-Berro, E., "The gravitational collapse of ONe electrondegenerate cores and white dwarfs: The role of ${ }^{24} \mathrm{Mg}$ and ${ }^{12} \mathrm{C}$ revisited", Astron. Astrophys., 435, 231-237, (2005). [DOI], [ADS], [arXiv:astro-ph/0502021]. (Cited on page 24.)

[128] Hachisu, I., "A versatile method for obtaining structures of rapidly rotating stars", Astrophys. J. Suppl. Ser., 61, 479-507, (1986). [DOI], [ADS]. (Cited on page 50.)

[129] Haehnelt, M.G., "Low-frequency gravitational waves from supermassive black holes", Mon. Not. R. Astron. Soc., 269, 199-208, (1994). [ADS]. (Cited on page 30.)

[130] Haehnelt, M.G., Natarajan, P. and Rees, M.J., "High-redshift galaxies, their active nuclei and central black holes", Mon. Not. R. Astron. Soc., 300, 817-827, (1998). [DOI], [ADS], [arXiv:astro-ph/9712259]. (Cited on page 29.)

[131] Haehnelt, M.G. and Rees, M.J., "The formation of nuclei in newly formed galaxies and the evolution of the quasar population", Mon. Not. R. Astron. Soc., 263, 168-178, (1993). [ADS]. (Cited on page 29.)

[132] Haensel, P., Levenfish, K.P. and Yakovlev, D.G., "Bulk viscosity in superfluid neutron star cores. III. Effects of $\Sigma^{-}$hyperons", Astron. Astrophys., 381, 1080-1089, (2002). [DOI], [ADS], [arXiv:astro-ph/0110575]. (Cited on pages 9 and 57.) 
[133] Hayashi, A., Eriguchi, Y. and Hashimoto, M., "On the Possibility of the Nonexplosive Core Contraction of Massive Stars: New Evolutionary Paths from Rotating White Dwarfs to Rotating Neutron Stars", Astrophys. J., 492, 286-297, (1998). [DOI], [ADS]. (Cited on page 33.)

[134] Hayashi, A., Eriguchi, Y. and Hashimoto, M., "On the Possibility of the Nonexplosive Core Contraction of Massive Stars. II. General Relativistic Analysis", Astrophys. J., 521, 376-381, (1999). [DOI], [ADS]. (Cited on page 33.)

[135] Heger, A., The presupernova evolution of rotating massive stars, Ph.D. Thesis, (Technische Universität München, Munich, 1998). (Cited on pages 37 and 49.)

[136] Heger, A., Fryer, C.L., Woosley, S.E., Langer, N. and Hartmann, D.H., "How Massive Single Stars End Their Life", Astrophys. J., 591, 288-300, (2003). [DOI], [ADS]. (Cited on pages 5, $13,16,26$, and 27.)

[137] Heger, A., Langer, N. and Woosley, S.E., "Presupernova Evolution of Rotating Massive Stars. I. Numerical Method and Evolution of the Internal Stellar Structure", Astrophys. J., 528, 368-396, (2000). [DOI], [ADS], [arXiv:astro-ph/9904132]. (Cited on page 52.)

[138] Heger, A., Woosley, S.E. and Spruit, H.C., "Presupernova Evolution of Differentially Rotating Massive Stars Including Magnetic Fields", Astrophys. J., 626, 350-363, (2005). [DOI], [ADS]. (Cited on pages 9, 37, and 49.)

[139] Herant, M., "The convective engine paradigm for the supernova explosion mechanism and its consequences", Phys. Rep., 256, 117-133, (1995). [DOI], [ADS]. (Cited on pages 18, 41, and 45.)

[140] Herant, M., Benz, W., Hix, W.R., Fryer, C.L. and Colgate, S.A., "Inside the supernova: A powerful convective engine", Astrophys. J., 435, 339-361, (1994). [DOI], [ADS]. (Cited on pages 13,18 , and 22.)

[141] Herwig, F., "Evolution of Asymptotic Giant Branch Stars", Annu. Rev. Astron. Astrophys., 43, 435-479, (2005). [DOI], [ADS]. (Cited on pages 24 and 26.)

[142] Hillebrandt, W., "Stellar Collapse and Supernova Explosions", in Pacini, F., ed., High Energy Phenomena around Collapsed Stars, Proceedings of the NATO Advanced Study Institute, Cargèse, Corsica, France, September 2-13, 1985, NATO Science Series, 195, pp. 73-104, (Reidel, Dordrecht; Boston, 1987). (Cited on pages 25, 26, and 43.)

[143] Ho, W.C.G. and Lai, D., " $r$-Mode Oscillations and Spin-down of Young Rotating Magnetic Neutron Stars", Astrophys. J., 543, 386-394, (2000). [DOI], [ADS], [arXiv:astro-ph/9912296]. (Cited on page 9.)

[144] Houck, J.C. and Chevalier, R.A., "Linear stability analysis of spherical accretion flows onto compact objects", Astrophys. J., 395, 592-603, (1992). [DOI], [ADS]. (Cited on pages 15 and 18.)

[145] Hough, J. and Rowan, S., "Laser interferometry for the detection of gravitational waves", $J$. Opt. A, 7, S257-S264, (2005). [DOI], [ADS]. (Cited on page 5.)

[146] Houser, J.L., "The effect of rotation on the gravitational radiation and dynamical stability of stiff stellar cores", Mon. Not. R. Astron. Soc., 299, 1069-1086, (1998). [DOI], [ADS]. (Cited on page 48.) 
[147] Houser, J.L., Centrella, J.M. and Smith, S.C., "Gravitational radiation from nonaxisymmetric instability in a rotating star", Phys. Rev. Lett., 72, 1314-1317, (1994). [DOI], [ADS]. (Cited on page 48.)

[148] Houser, J.L., Centrella, J.M. and Smith, S.C., "Gravitational radiation from rotational instabilities in compac stellar cores with stiff equations of state", Phys. Rev. D, 54, 7278-7297, (1996). [DOI], [ADS]. (Cited on page 48.)

[149] Hughes, S.A., "Untangling the merger history of massive black holes with LISA", Mon. Not. R. Astron. Soc., 331, 805-816, (2002). [DOI], [ADS], [arXiv:astro-ph/0108483]. (Cited on page 61.)

[150] Hughes, S.A., Márka, S., Bender, P.L. and Hogan, C.J., "New physics and astronomy with the new gravitational-wave observatories", in Graf, N., ed., Proceedings of Snowmass 2001, The Future of Particle Physics, 30 June-21 July 2001, Snowmass Village, Colorado, C010630, p. P402, (SLAC eConf, Stanford, 2001). URL (accessed 15 October 2001):

http://www.slac.stanford.edu/econf/C010630/proceedings.shtml. (Cited on page 5.)

[151] Iben Jr, I., "Massive stars in quasi-static equilibrium", Astrophys. J., 138, 1090-1096, (1963). [DOI], [ADS]. (Cited on page 30.)

[152] Iben Jr, I. and Renzini, A., "Asymptotic Giant Branch Evolution and Beyond", Annu. Rev. Astron. Astrophys., 21, 271-342, (1983). [DOI], [ADS]. (Cited on page 26.)

[153] Imamura, J.N. and Durisen, R.H., "The Dominance of Dynamic Barlike Instabilities in the Evolution of a Massive Stellar Core Collapse That 'Fizzles"', Astrophys. J., 549, 1062-1075, (2001). [DOI], [ADS]. (Cited on page 33.)

[154] Imamura, J.N., Durisen, R.H. and Pickett, B.K., "Nonaxisymmetric Dynamic Instabilities of Rotating Polytropes. II. Torques, Bars, and Mode Saturation with Applications to Protostars and Fizzlers", Astrophys. J., 528, 946-964, (2000). [DOI], [ADS]. (Cited on page 48.)

[155] Janka, H.-T., "Supermassive Stars: Fact or Fiction?", in Chui, C.K., Siuniaev, R.A. and Churazov, E., eds., Lighthouses of the Universe: The Most Luminous Celestial Objects and Their Use for Cosmology, Proceedings of the MPA/ESO/MPE/USM Joint Astronomy Conference, held in Garching, Germany, 6-10 August 2001, ESO Astrophysics Symposia, pp. 357-368, (Springer, Berlin; New York, 2002). [DOI], [ADS], [astro-ph/0202028]. (Cited on pages 29 and 30.)

[156] Janka, H.-T., Langanke, K., Marek, A., Martinez-Pinedo, G. and Müller, B., "Theory of Core-Collapse Supernovae", Phys. Rep., 442, 38-74, (2007). [DOI], [ADS]. (Cited on page 13.)

[157] Jenet, F.A. and Prince, T.A., "Detection of variable frequency signals using a fast chirp transform", Phys. Rev. D, 62, 122001, 1-10, (2000). [DOI], [ADS], [arXiv:gr-qc/0012029]. (Cited on page 49.)

[158] Jones, P.B., "Bulk viscosity of neutron-star matter", Phys. Rev. D, 64, 084003, 1-7, (2001). [DOI], [ADS]. (Cited on pages 9 and 57.)

[159] Jones, P.B., "Comment on 'Gravitational radiation instability in hot young neutron stars"', Phys. Rev. Lett., 86, 1384, (2001). [DOI], [ADS]. (Cited on pages 9 and 57.)

[160] Keil, W., Janka, H.-T. and Müller, E., "Ledoux Convection in Protoneutron Stars-A Clue to Supernova Nucleosynthesis?", Astrophys. J., 473, 111-114, (1996). [DOI], [ADS]. (Cited on page 22.) 
[161] Kitaura, F.S., Janka, H.-T. and Hillebrandt, W., "Explosions of O-Ne-Mg cores, the Crab supernova, and subluminous type II-P supernovae", Astron. Astrophys., 450, 345-350, (2006). [DOI], [ADS]. (Cited on pages 24 and 25.)

[162] Kokkotas, K.D. and Schmidt, B., "Quasi-Normal Modes of Stars and Black Holes", Living Rev. Relativity, 2, lrr-1999-2, (1999). URL (accessed 18 May 2009):

http://www.livingreviews.org/lrr-1999-2. (Cited on pages 7 and 60.)

[163] Kormendy, J., "Supermassive Black Holes in Disk Galaxies", in Funes, J.G. and Corsini, E.M., eds., Galaxy Disks and Disk Galaxies, Proceedings of a conference sponsored by the Vatican Observatory, held at the Pontifical Gregorian University in Rome, Italy, 12 - 16 June 2000, ASP Conference Series, 230, pp. 247-256, (Astronomical Society of the Pacific, San Francisco, 2001). [ADS]. (Cited on page 29.)

[164] Kotake, K., Iwakami, W., Ohnishi, N. and Yamada, S., "Gravitational Radiation from Standing Accretion Shock Instability in Core-Collapse Supernovae", Astrophys. J. Lett., 697, L133-L136, (2009). [DOI], [ADS]. (Cited on page 18.)

[165] Kotake, K., Ohnishi, N. and Yamada, S., "Gravitational Radiation from Standing Accretion Shock Instability in Core-Collapse Supernovae", Astrophys. J., 655, 406-415, (2007). [DOI], [ADS]. (Cited on pages $6,7,41,45$, and 54.)

[166] Kotake, K., Sato, K. and Takahashi, K., "Explosion mechanism, neutrino burst and gravitational wave in core-collapse supernovae", Rep. Prog. Phys., 69, 971-1143, (2006). [DOI], [ADS]. (Cited on pages 54 and 56.)

[167] Kotake, K., Sato, K. and Takahashi, K., "Gravitational Radiation from Standing Accretion Shock Instability in Core-Collapse Supernovae", Rep. Prog. Phys., 69, 971-1143, (2006). [ADS]. (Cited on pages 5, 32, and 33.)

[168] Kotake, K., Yamada, S. and Sato, K., "Gravitational radiation from axisymmetric rotational core collapse", Phys. Rev. D, 68, 044023, (2003). [DOI], [ADS]. (Cited on pages 33, 35, and 36.)

[169] Kotake, K., Yamada, S., Sato, K., Sumiyoshi, K., Ono, H. and Suzuki, H., "Gravitational radiation from rotational core collapse: Effects of magnetic fields and realistic equations of state", Phys. Rev. D, 69, 124004, 1-11, (2004). [DOI], [ADS]. (Cited on page 36.)

[170] Kusenko, A. and Segre, G., "Pulsar Velocities and Neutrino Oscillations", Phys. Rev. Lett., 77, 4872-4875, (1996). [DOI], [ADS]. (Cited on pages 22 and 23.)

[171] Lai, D., "Secular bar-mode evolution and gravitational waves from neutron stars", in Centrella, J.M., ed., Astrophysical Sources for Ground-based Gravitational Wave Detectors, Philadelphia, PA, USA, 30 October-1 November 2000, AIP Conference Proceedings, 575, pp. 246-257, (American Institute of Physics, Melville, N.Y., 2001). [DOI], [ADS]. (Cited on pages 48 and 49.)

[172] Lai, D. and Goldreich, P., "Growth of Perturbations in Gravitational Collapse and Accretion", Astrophys. J., 535, 402-411, (2000). [DOI], [ADS], [arXiv:astro-ph/9906400]. (Cited on page 41.)

[173] Lai, D. and Shapiro, S.L., "Gravitational Radiation from Rapidly Rotating Nascent Neutron Stars", Astrophys. J., 442, 259-272, (1995). [DOI], [ADS], [arXiv:astro-ph/9408053]. (Cited on pages 48 and 49.) 
[174] Leaver, E.W., "An analytic representation for the quasi-normal modes of Kerr black holes", Proc. R. Soc. London, Ser. A, 402, 285-298, (1985). [ADS]. (Cited on page 63.)

[175] LeBlanc, J.M. and Wilson, J.R., "An analytic representation for the quasi-normal modes of Kerr black holes", Astrophys. J., 161, 541-551, (1985). [DOI], [ADS]. (Cited on pages 18 and 27.)

[176] Li, H., Finn, J.M., Lovelace, R.V.E. and Colgate, S.A., "Rossby Wave Instability of Thin Accretion Disks. II. Detailed Linear Theory", Astrophys. J., 533, 1023-1034, (2000). [DOI], [ADS]. (Cited on page 53.)

[177] "LIGO Laboratory Home Page", project homepage, California Institute of Technology. URL (accessed 4 October 2002): http://www.ligo.caltech.edu. (Cited on page 5.)

[178] Lindblom, L. and Owen, B.J., "Effect of hyperon bulk viscosity on neutron-star $r$-modes", Phys. Rev. D, 65, 063006, 1-15, (2002). [DOI], [ADS], [arXiv:astro-ph/0110558]. (Cited on pages 9 and 57.)

[179] Lindblom, L., Owen, B.J. and Morinsk, S.M., "Gravitational Radiation Instability in Hot Young Neutron Stars", Phys. Rev. Lett., 80, 4843-4846, (1998). [DOI], [ADS], [arXiv:grqc/9803053]. (Cited on page 57.)

[180] Lindblom, L., Tohline, J.E. and Vallisneri, M., "Nonlinear Evolution of the $r$-Modes in Neutron Stars", Phys. Rev. Lett., 86, 1152-1155, (2001). [DOI], [ADS], [arXiv:astro-ph/0010653]. (Cited on page 57.)

[181] Lindblom, L., Tohline, J.E. and Vallisneri, M., "Numerical evolutions of nonlinear $r$-modes in neutron stars", Phys. Rev. D, 65, 084039, 1-15, (2002). [DOI], [ADS], [arXiv:astro-ph/0109352]. (Cited on pages 9 and 57.)

[182] "LISA: Laser Interferometer Space Antenna", project homepage, NASA. URL (accessed 4 October 2002):

http://lisa.nasa.gov. (Cited on page 5.)

[183] Liu, Y.T., "Dynamical instability of new-born neutron stars as sources of gravitational radiation", Phys. Rev. D, 65, 124003, 1-14, (2002). [DOI], [ADS], [arXiv:gr-qc/0109078]. (Cited on pages 50 and 51.)

[184] Liu, Y.T. and Lindblom, L., "Models of rapidly rotating neutron stars: remnants of accretioninduced collapse", Mon. Not. R. Astron. Soc., 324, 1063-1073, (2001). [DOI], [ADS]. (Cited on pages 48,50 , and 51 .)

[185] Liu, Y.T., Shapiro, S.L. and Stephens, B.C., "Magnetorotational collapse of very massive stars to black holes in full general relativity", Phys. Rev. D, 76, 084017, (2007). [DOI], [ADS]. (Cited on pages 30 and 59.)

[186] Loeb, A. and Rasio, F.A., "Collapse of Primordial Gas Clouds and the Formation of Quasar Black Holes", Astrophys. J., 432, 52-61, (1994). [DOI], [ADS], [arXiv:astro-ph/9401026]. (Cited on page 29.)

[187] Loveridge, L.C., "Gravitational waves from a pulsar kick caused by neutrino conversions", Phys. Rev. D, 69, 024008, 1-8, (2004). [DOI], [ADS]. (Cited on page 54.)

[188] Macchetto, F.D., "Supermassive Black Holes and Galaxy Morphology", Astrophys. Space Sci., 269, 269-291, (1999). [DOI], [arXiv:astro-ph/9910089]. (Cited on page 29.) 
[189] MacFadyen, A.I. and Woosley, S.E., "Collapsars: Gamma-Ray Bursts and Explosions in 'Failed Supernovae"', Astrophys. J., 524, 262-289, (1999). [DOI], [ADS], [arXiv:astroph/9810274]. (Cited on page 27.)

[190] MacFadyen, A.I., Woosley, S.E. and Heger, A., "Supernovae, Jets, and Collapsars", Astrophys. J., 550, 410-425, (2001). [DOI], [ADS]. (Cited on page 27.)

[191] Maeda, K. et al., "The Unique Type Ib Supernova 2005bf at Nebular Phases: A Possible Birth Event of a Strongly Magnetized Neutron Star", Astrophys. J., 666, 1069-1082, (2007). [DOI], [ADS]. (Cited on page 22.)

[192] Managan, R.A., "On the Secular Instability of Axisymmetric Rotating Stars to Gravitational Radiation Reaction", Astrophys. J., 294, 463-473, (1985). [DOI], [ADS]. (Cited on page 48.)

[193] Mannucci, F., Della Valle, M. and Panagia, N., "How many supernovae are we missing at high redshift", Mon. Not. R. Astron. Soc., 377, 1229-1235, (2007). [DOI], [arXiv:astro-ph/0702355]. (Cited on pages 19 and 20.)

[194] Marck, J.-A. and Bonazzola, S., "Gravitational radiation from three-dimensional gravitational stellar core collapse", in D'Inverno, R., ed., Approaches to Numerical Relativity, Proceedings of the International Workshop on Numerical Relativity, Southampton, England, 16-20 December 1991, p. 247, (Cambridge University Press, Cambridge, 1992). (Cited on page 51.)

[195] Marek, A. and Janka, H.-T., "Delayed Neutrino-Driven Supernova Explosions Aided by the Standing Accretion-Shock Instability", Astrophys. J., 694, 664-696, (2009). [DOI], [ADS]. (Cited on pages 18, 19, 22, 45, and 54.)

[196] Marek, A., Janka, H.-T. and Müller, E., "Equation-of-state dependent features in shockoscillation modulated neutrino and gravitational-wave signals from supernovae", Astron. Astrophys., 496, 475-494, (2009). [DOI], [ADS]. (Cited on pages 18 and 45.)

[197] Miyaji, S. and Nomoto, K., "On the collapse of 8-10 $M_{\odot}$ stars due to electron capture", Astrophys. J., 318, 307-315, (1987). [DOI], [ADS]. (Cited on page 26.)

[198] Mochkovitch, R. and Livio, M., "The coalescence of white dwarfs and type I supernovae", Astron. Astrophys., 209, 111-118, (1989). [ADS]. (Cited on page 25.)

[199] Mochkovitch, R. and Livio, M., "The coalescence of white dwarfs and type I supernovae. The merged configuration", Astron. Astrophys., 236, 378-384, (1990). [ADS]. (Cited on page 25.)

[200] Moe, M. and De Marco, O., "Do Most Planetary Nebulae Derive from Binaries? I. Population Synthesis Model of the Galactic Planetary Nebula Population Produced by Single Stars and Binaries", Astrophys. J., 650, 916-932, (2006). [DOI], [ADS]. (Cited on page 24.)

[201] Mönchmeyer, R., Schäfer, G., Müller, E. and Kates, R.E., "Gravitational waves from the collapse of rotating stellar cores", Astron. Astrophys., 246, 417-440, (1991). [ADS]. (Cited on pages $6,10,32,33$, and 35 .)

[202] Moncrief, V., "Reduction of the Einstein equations in $2+1$ dimensions to a Hamiltonian system over Teichmüller space", J. Math. Phys., 30, 2907-2914, (1989). [DOI], [ADS]. (Cited on page 12.)

[203] Motl, P.M., Tohline, J.E. and Frank, J., "Numerical Methods for the Simulation of Dynamical Mass Transfer in Binaries", Astrophys. J. Suppl. Ser., 138, 121-148, (2002). [DOI], [ADS]. (Cited on page 25.) 
[204] Müller, E., "Gravitational Radiation from Collapsing Rotating Stellar Cores", Astron. Astrophys., 114, 53-59, (1982). [ADS]. (Cited on pages 6, 32, and 33.)

[205] Müller, E., "Gravitational waves from core collapse supernovae", in Marck, J.-A. and Lasota, J.-P., eds., Relativistic Gravitation and Gravitational Radiation, Proceedings of the Les Houches School of Physics, held in Les Houches, Haute Savoie, 26 September-6 October, 1995, Cambridge Contemporary Astrophysics, pp. 273-308, (Cambridge University Press, Cambridge, 1997). (Cited on page 5.)

[206] Müller, E., "Simulation of Astrophysical Fluid Flow", in LeVeque, R.J., Mihalas, D., Dorfi, E.A., Müller, E., Steiner, O. and Gautschy, A., eds., Computational Methods for Astrophysical Fluid Flow, Lecture Notes of the Saas-Fee Advanced Course 27, Les Diablerets, Switzerland, March 3-8, 1997, Saas-Fee Advanced Courses, 27, pp. 343-494, (Springer, Berlin; New York, 1998). [ADS]. (Cited on pages 5 and 41.)

[207] Müller, E. and Hillebrandt, W., "The Collapse of Rotating Stellar Cores", Astron. Astrophys., 103, 358-366, (1981). [ADS]. (Cited on page 32.)

[208] Müller, E. and Janka, H.-T., "Gravitational radiation from convective instabilities in Type II supernova explosions", Astron. Astrophys., 317, 140-163, (1997). [ADS]. (Cited on pages 10, $11,41,43,44$, and 45.$)$

[209] Müller, E., Rampp, M., Buras, R., Janka, H.-T. and Shoemaker, D.H., "Toward Gravitational Wave Signals from Realistic Core-Collapse Supernova Models", Astrophys. J., 603, 221-230, (2004). [DOI], [ADS]. (Cited on pages 36, 43, 45, 54, and 55.)

[210] Müller, E., Rózyczka, M. and Hillebrandt, W., "Stellar Collapse: Adiabatic Hydrodynamics and Shock Wave Propagation", Astron. Astrophys., 81, 288-292, (1980). [ADS]. (Cited on page 33.)

[211] Murphy, J.W. and Burrows, A., "Criteria for Core-Collapse Supernova Explosions by the Neutrino Mechanism", Astrophys. J., 688, 1159-1175, (2008). [DOI], [ADS]. (Cited on page 13.)

[212] Murphy, J.W., Burrows, A. and Heger, A., "Pulsational Analysis of the Cores of Massive Stars and Its Relevance to Pulsar Kicks", Astrophys. J., 615, 460-474, (2004). [DOI], [ADS]. (Cited on page 41.)

[213] Murphy, J.W., Ott, C.D. and Burrows, A., "A Model for Gravitational Wave Emission from Neutrino-Driven Core-Collapse Supernovae", Astrophys. J., 707, 1173-1190, (2009). [DOI], [ADS]. (Cited on page 41.)

[214] Nagar, A. and Rezzolla, L., "Gauge-invariant non-spherical metric perturbations of Schwarzschild black-hole spacetimes", Class. Quantum Grav., 22, R167-R192, (2005). [DOI], [ADS]. (Cited on page 12.)

[215] Nagar, A., Zanotti, O., Font, J.A. and Rezzolla, L., "Accretion-driven gravitational radiation from nonrotating compact objects: Infalling quadrupolar shells", Phys. Rev. D, 69, 124028, (2004). [DOI], [ADS]. (Cited on page 60.)

[216] Nagar, A., Zanotti, O., Font, J.A. and Rezzolla, L., "Accretion-induced quasinormal mode excitation of a Schwarzschild black hole", Phys. Rev. D, 75, 044016, (2007). [DOI], [ADS]. (Cited on page 60.) 
[217] Nakazato, K., Sumiyoshi, K. and Yamada, S., "Gravitational Collapse and Neutrino Emission of Population III Massive Stars", Astrophys. J., 645, 519-533, (2006). [DOI], [ADS]. (Cited on pages 15 and 27.)

[218] Nakazato, K., Sumiyoshi, K. and Yamada, S., "Numerical Study of Stellar Core Collapse and Neutrino Emission: Probing the Spherically Symmetric Black Hole Progenitors with 3-30 $M_{\odot}$ Iron Cores", Astrophys. J., 666, 1140-1151, (2007). [DOI], [ADS]. (Cited on pages 15 and 27.)

[219] Narayan, R., Paczyński, B. and Piran, T., "Gamma-Ray Bursts as the Death Throes of Massive Binary Stars", Astrophys. J. Lett., 395, L83-L86, (1992). [DOI], [ADS]. (Cited on pages 18 and 27.)

[220] Nazin, S.N. and Postnov, K.A., "High neutron star birth velocities and gravitational radiation during supernova explosions", Astron. Astrophys., 317, L79-L81, (1997). [ADS], [arXiv:astroph/9701073]. (Cited on page 41.)

[221] New, K.C.B., Centrella, J.M. and Tohline, J.E., "Gravitational waves from long-duration simulations of the dynamical bar instability", Phys. Rev. D, 62, 064019, 1-16, (2000). [DOI], [ADS], [arXiv:astro-ph/9911525]. (Cited on pages 48, 50, and 51.)

[222] New, K.C.B. and Shapiro, S.L., "Evolution of Differentially Rotating Supermassive Stars to the Onset of Bar Instability", Astrophys. J., 548, 439-446, (2001). [DOI], [ADS], [arXiv:astroph/0010172]. (Cited on page 30.)

[223] New, K.C.B. and Shapiro, S.L., "The formation of supermassive black holes and the evolution of supermassive stars", Class. Quantum Grav., 18, 3965-3975, (2001). [DOI], [ADS], [arXiv:astro-ph/0009095]. (Cited on page 30.)

[224] Nomoto, K. and Kondo, Y., "Conditions for accretion-induced collapse of white dwarfs", Astrophys. J. Lett., 367, L19-L22, (1991). [DOI], [ADS]. (Cited on page 24.)

[225] Novikov, I.D., "Gravitational radiation from a star collapsing into a disk", Sov. Astron., 19, 398-399, (1976). [ADS]. (Cited on page 32.)

[226] Obergaulinger, M., Aloy, M.A., Dimmelmeier, H. and Müller, E., "Axisymmetric simulations of magnetorotational core collapse: approximate inclusion of general relativistic effects", Astron. Astrophys., 457, 209-222, (2006). [DOI], [ADS]. (Cited on pages 36 and 37.)

[227] Obergaulinger, M., Aloy, M.A. and Müller, E., "Axisymmetric simulations of magnetorotational core collapse: dynamics and gravitational wave signal", Astron. Astrophys., 450, 1107-1134, (2006). [DOI], [ADS]. (Cited on pages 36 and 37.)

[228] Ott, C.D., "The gravitational-wave signature of core-collapse supernovae", Class. Quantum Grav., 26, 063001, (2009). [DOI], [ADS]. (Cited on pages 5, 7, 22, 32, 33, 45, 54, and 67.)

[229] Ott, C.D., Burrows, A., Dessart, L. and Livne, E., "A New Mechanism for GravitationalWave Emission in Core-Collapse Supernovae", Phys. Rev. Lett., 96, 201102, (2006). [DOI], [ADS]. (Cited on pages 18, 23, and 45.)

[230] Ott, C.D., Burrows, A., Livne, E. and Walder, R., "Gravitational Waves from Axisymmetric, Rotating Stellar Core Collapse", Astrophys. J., 600, 834-864, (2004). [DOI], [ADS]. (Cited on pages 35 and 36. .) 
[231] Ott, C.D., Burrows, A., Thompson, T.A., Livne, E. and Walder, R., "The Spin Periods and Rotational Profiles of Neutron Stars at Birth", Astrophys. J., 164, 130-155, (2006). [DOI], [ADS]. (Cited on page 49.)

[232] Ott, C.D., Dimmelmeier, H., Marek, A., Janka, H.-T., Hawke, I., Zink, B. and Schnetter, E., "3D Collapse of Rotating Stellar Iron Cores in General Relativity Including Deleptonization and a Nuclear Equation of State", Phys. Rev. Lett., 98, 261101, (2007). [DOI], [ADS]. (Cited on pages $6,36,40$, and 48 .)

[233] Ott, C.D., Dimmelmeier, H., Marek, A., Janka, H.-T., Zink, B., Hawke, I. and Schnetter, E., "Rotating collapse of stellar iron cores in general relativity", Class. Quantum Grav., 24, S139-S154, (2007). [DOI], [ADS]. (Cited on pages 36, 40, and 48.)

[234] Ott, C.D., Ou, S., Tohline, J.E. and Burrows, A., "One-armed Spiral Instability in a Low$T /|W|$ Postbounce Supernova Core", Astrophys. J., 625, L119-L122, (2005). [DOI], [ADS]. (Cited on pages 49 and 53.)

[235] Ou, S. and Tohline, J.E., "Unexpected Dynamical Instabilities in Differentially Rotating Neutron Stars", Astrophys. J., 651, 1068-1078, (2006). [DOI], [ADS]. (Cited on pages 49 and 53.)

[236] Ou, S., Tohline, J.E. and Lindblom, L., "Nonlinear Development of the Secular Bar-Mode Instability in Rotating Neutron Stars", Astrophys. J., 617, 490-499, (2004). [DOI], [ADS]. (Cited on pages 48 and 49.)

[237] Pickett, B.K., Durisen, R.H. and Davis, G.A., "The Dynamic Stability of Rotating Protostars and Protostellar Disks. I. The Effects of the Angular Momentum Distribution", Astrophys. J., 458, 714-738, (1996). [DOI], [ADS]. (Cited on pages 48, 50, and 51.)

[238] Piran, T. and Stark, R.F., "Numerical relativity, rotating gravitational collapse, and gravitational radiation", in Centrella, J.M., ed., Dynamical Spacetimes and Numerical Relativity, Proceedings of a workshop held at Drexel University, October 7-11, 1985, pp. 40-73, (Cambridge University Press, Cambridge; New York, 1986). [ADS]. (Cited on page 32.)

[239] Piro, A.L. and Pfahl, E., "Fragmentation of Collapsar Disks and the Production of Gravitational Waves", Astrophys. J., 658, 1173-1176, (2007). [DOI], [ADS]. (Cited on page 8.)

[240] Podsiadlowski, Ph., Mazzali, P.A., Nomoto, K., Lazzati, D. and Cappellaro, E., "The Rates of Hypernovae and Gamma-Ray Brusts: Implications for Their Progenitors", Astrophys. J., 607, L17-L20, (2004). [DOI], [ADS]. (Cited on pages 9 and 49.)

[241] Poelarends, A.J.T., Herwig, F., Langer, N. and Heger, A., "The Supernova Channel of Super-AGB Stars", Astrophys. J., 675, 614-625, (2008). [DOI], [ADS]. (Cited on pages 24 and 26.)

[242] Popham, R., Woosley, S.E. and Fryer, C.L., "Hyperaccreting Black Holes and Gamma-Ray Bursts", Astrophys. J., 518, 356-374, (1999). [DOI], [ADS]. (Cited on pages 8 and 27.)

[243] Porter, D.H. and Woodward, P.R., "Using PPM to Model Turbulent Stellar Convection", in Grinstein, F., Margolin, L. and Rider, W., eds., Implicit Large Eddy Simulation: Computing Turbulent Fluid Dynamics, (Cambridge University Press, Los Alamos, NM, 2006). [ADS]. (Cited on page 19.)

[244] Proga, D., MacFadyen, A.I., Armitage, P.J. and Begelman, M.C., "Axisymmetric Magnetohydrodynamic Simulations of the Collapsar Model for Gamma-Ray Bursts", Astrophys. J. Lett., 599, L5-L8, (2003). [DOI], [ADS]. (Cited on page 27.) 
[245] Rampp, M., Müller, E. and Ruffert, M., "Simulations of non-axisymmetric rotational core collapse", Astron. Astrophys., 332, 969-983, (1998). [arXiv:astro-ph/9781112]. (Cited on pages $6,8,9,36,49,51,52$, and 53.$)$

[246] Rees, M.J., "Astrophysical Evidence for Black Holes", in Wald, R.M., ed., Black Holes and Relativistic Stars, Proceedings of the Symposium dedicated to the memory of Subrahmanyan Chandrasekhar, held in Chicago, December 14-15, 1996, pp. 79-101, (University of Chicago Press, Chicago; London, 1998). [ADS]. (Cited on page 29.)

[247] Rezzolla, L., "Relativistic Astrophysics movies at SISSA", personal homepage, SISSA / ISAS, (2002). URL (accessed 4 October 2002): http://people.sissa.it/ rezzolla/movies.html. (Cited on page 58.)

[248] Rezzolla, L., Lamb, F.K., Marković, D. and Shapiro, S.L., "Properties of $r$ modes in rotating magnetic neutron stars. I. Kinematic secular effects and magnetic evolution", Phys. Rev. D, 64, 104013, 1-12, (2001). [DOI], [arXiv:gr-qc/0107061]. (Cited on pages 9 and 57.)

[249] Rezzolla, L., Lamb, F.L., Marković, D. and Shapiro, S.L., "Properties of $r$ modes in rotating magnetic neutron stars. II. Evolution of the $r$ modes and stellar magnetic field", Phys. Rev. D, 64, 104014, 1-13, (2001). [DOI], [arXiv:gr-qc/0107062]. (Cited on pages 9 and 57.)

[250] Rockefeller, G., Fryer, C.L. and Li, H., "Collapsars in Three Dimensions", arXiv e-print, (2006). [arXiv:astro-ph/0608028]. (Cited on pages 27, 59, and 64.)

[251] Ruffini, R. and Wheeler, J.A., "Relativistic Cosmology from Space Platforms", in Hardy, V. and Moore, H., eds., Proceedings of the Conference on Space Physics, pp. 45-174, (ESRO, Paris, 1971). (Cited on pages 6 and 32.)

[252] Sá, P.M. and Tomé, B., "Gravitational waves from r-modes", Astrophys. Space Sci., 308, 557-561, (2007). [DOI], [ADS]. (Cited on page 57.)

[253] Saenz, R.A. and Shapiro, S.L., "Gravitational Radiation from Stellar Collapse: Ellipsoidal Models", Astrophys. J., 221, 286-303, (1978). [DOI], [ADS]. (Cited on pages 6 and 32.)

[254] Saenz, R.A. and Shapiro, S.L., "Gravitational and Neutrino Radiation from Stellar Core Collapse: Improved Ellipsoidal Model Calculations", Astrophys. J., 229, 1107-1125, (1979). [DOI], [ADS]. (Cited on page 32.)

[255] Saenz, R.A. and Shapiro, S.L., "Gravitational Radiation from Stellar Core Collapse. III. Damped Ellipsoidal Oscillations", Astrophys. J., 244, 1033-1038, (1981). [DOI], [ADS]. (Cited on page 32.)

[256] Saijo, M., "The Collapse of Differentially Rotating Supermassive Stars: Conformally Flat Simulations", Astrophys. J., 615, 866-879, (2004). [DOI], [ADS]. (Cited on page 61.)

[257] Saijo, M., "Dynamical bar instability in a relativistic rotational collapse", Phys. Rev. D, 71, 104038, (2005). [DOI], [ADS]. (Cited on page 49.)

[258] Saijo, M., Baumgarte, T.W. and Shapiro, S.L., "One-armed Spiral Instability in Differentially Rotating Stars", Astrophys. J., 595, 352-364, (2003). [DOI], [ADS]. (Cited on pages 49 and 53.)

[259] Saijo, M., Baumgarte, T.W., Shapiro, S.L. and Shibata, M., "Collapse of a rotating supermassive star to a supermassive black hole: Post-Newtonian simulations", Astrophys. J., 569, 349-361, (2002). [DOI], [arXiv:astro-ph/0202112]. (Cited on pages 61 and 62.) 
[260] Saijo, M. and Yoshida, S., "Low $T /|W|$ dynamical instability in differentially rotating stars: diagnosis with canonical angular momentum", Mon. Not. R. Astron. Soc., 368, 1429-1442, (2006). [DOI], [ADS]. (Cited on pages 49 and 53.)

[261] Salpeter, E.E., "Energy and pressure of a zero-temperature plasma", Astrophys. J., 134, 669-682, (1961). [DOI], [ADS]. (Cited on page 50.)

[262] Sanders, R.H., "The Effects of Stellar Collisions in Dense Stellar Systems", Astrophys. J., 162, 791-809, (1970). [DOI], [ADS]. (Cited on page 29.)

[263] Scheck, L., Janka, H.-T., Foglizzo, T. and Kifonidis, K., "Multidimensional supernova simulations with approximative neutrino transport. II. Convection and the advective-acoustic cycle in the supernova core", Astron. Astrophys., 477, 931-952, (2008). [DOI], [ADS]. (Cited on page 18.)

[264] Scheck, L., Kifonidis, K., Janka, H.-T. and Müller, E., "Multidimensional supernova simulations with approximative neutrino transport. I. Neutron star kicks and the anisotropy of neutrino-driven explosions in two spatial dimensions", Astron. Astrophys., 457, 963-986, (2006). [DOI], [ADS]. (Cited on page 18.)

[265] Scheidegger, S., Fischer, T., Whitehouse, S.C. and Liebendörfer, M., "Gravitational waves from 3D MHD core collapse simulations", Astron. Astrophys., 490, 231-241, (2008). [DOI], [ADS]. (Cited on page 18.)

[266] Scheidegger, S., Fischer, T., Whitehouse, S.C. and Liebendörfer, M., "Gravitational waves from supernova matter", Class. Quantum Grav., 27, 114101, (2010). [DOI], [ADS]. (Cited on page 36.)

[267] Scheidegger, S., Käppeli, R., Whitehouse, S.C., Fischer, T. and Liebendörfer, M., "The influence of model parameters on the prediction of gravitational wave signals from stellar core collapse", Astron. Astrophys., 514, A51, (2010). [DOI], [ADS]. (Cited on page 36.)

[268] Schenk, A.K., Arras, P., Flanagan, É.É., Teukolsky, S.A. and Wasserman, I., "Nonlinear mode coupling in rotating stars and the $r$-mode instability in neutron stars", Phys. Rev. D, 65, 024001, 1-43, (2002). [arXiv:gr-qc/0101092]. (Cited on page 57.)

[269] Schutz, B.F., "Gravitational Wave Astronomy", Class. Quantum Grav., 16, A131-A156, (1999). [DOI], [ADS]. (Cited on page 5.)

[270] Segretain, L., Chabrier, G. and Mochkovitch, R., "The Fate of Merging White Dwarfs", Astrophys. J., 481, 355-362, (1997). [DOI], [ADS]. (Cited on page 25.)

[271] Seidel, E. and Moore, T., "Gravitational radiation from realistic relativistic stars: Odd-parity fluid perturbations", Phys. Rev. D, 35, 2287-2296, (1987). [DOI], [ADS]. (Cited on page 32.)

[272] Seidel, E. and Moore, T., "Gravitational radiation from perturbations of stellar core collapse models", in Evans, C.R., Finn, L.S. and Hobill, D.W., eds., Frontiers in Numerical Relativity, pp. 146-162, (Cambridge University Press, Cambridge; New York, 1988). (Cited on page 32.)

[273] Shapiro, S.L., "Gravitational Radiation from Stellar Collapse: The Initial Burst", Astrophys. $J .$, 214, 566-575, (1977). [DOI], [ADS]. (Cited on page 32.)

[274] Shapiro, S.L. and Lightman, A.P., "Rapidly Rotating, Post-Newtonian Neutron Stars", Astrophys. J., 207, 263-278, (1976). [DOI], [ADS]. (Cited on page 33.) 
[275] Shapiro, S.L. and Teukolsky, S.A., "Gravitational Collapse of Supermassive Stars to Black Holes: Numerical Solution of the Einstein Equations", Astrophys. J. Lett., 234, L177-L181, (1979). [DOI], [ADS]. (Cited on page 60.)

[276] Shapiro, S.L. and Teukolsky, S.A., Black Holes, White Dwarfs, and Neutron Stars: The Physics of Compact Objects, (Wiley, New York, 1983). [Google Books]. (Cited on pages 29, 30 , and 48.)

[277] Shibata, M., "Fully general relativistic simulation of coalescing binary neutron stars: Preparatory tests", Phys. Rev. D, 60, 104052, 1-25, (1999). [DOI], [ADS]. (Cited on page 12.)

[278] Shibata, M., Karino, S. and Eriguchi, Y., "Dynamical instability of differentially rotating stars", Mon. Not. R. Astron. Soc., 334, L27-L31, (2002). [DOI], [ADS]. (Cited on pages 9, 49, and 53.)

[279] Shibata, M., Karino, S. and Eriguchi, Y., "Dynamical instability of differentially rotating stars", Mon. Not. R. Astron. Soc., 343, 619-626, (2003). [DOI], [ADS]. (Cited on pages 9, 49, and 53.)

[280] Shibata, M. and Nakamura, T., "Evolution of three-dimensional gravitational waves: Harmonic slicing case", Phys. Rev. D, 52, 5428-5444, (1995). [DOI], [ADS]. (Cited on page 12.)

[281] Shibata, M. and Sekiguchi, Y., "Gravitational waves from axisymmetric rotating stellar core collapse to a neutron star in full general relativity", Phys. Rev. D, 69, 084024, 1-16, (2004). [DOI], [ADS]. (Cited on page 37.)

[282] Shibata, M. and Sekiguchi, Y., "Three-dimensional simulations of stellar core collapse in full general relativity: Nonaxisymmetric dynamical instabilities", Phys. Rev. D, 71, 024014, 1-32, (2005). [DOI], [ADS]. (Cited on pages 8, 12, and 49.)

[283] Shibata, M. and Sekiguchi, Y.-I., "Three-dimensional simulations of stellar core collapse in full general relativity: Nonaxisymmetric dynamical instabilities", Phys. Rev. D, 71, 024014, (2005). [DOI], [ADS]. (Cited on page 37.)

[284] Shibata, M. and Shapiro, S.L., "Collapse of a Rotating Supermassive Star to a Supermassive Black Hole: Fully Relativistic Simulations", Astrophys. J. Lett., 572, L39-L43, (2002). [DOI], [ADS]. (Cited on page 61.)

[285] Shibata, M., Shapiro, S.L. and Uryū, K., "Equilibrium and stability of supermassive stars in binary systems", Phys. Rev. D, 64, 024004, 1-14, (2001). [DOI], [ADS], [arXiv:astroph/0104408]. (Cited on page 63.)

[286] Siess, L., "Evolution of massive AGB stars. I. Carbon burning phase", Astron. Astrophys., 448, 717-729, (2006). [DOI], [ADS]. (Cited on pages 24 and 26.)

[287] Siess, L., "Evolution of massive AGB stars. II. model properties at non-solar metallicity and the fate of Super-AGB stars", Astron. Astrophys., 476, 893-909, (2007). [DOI], [ADS]. (Cited on pages 24 and 26.)

[288] Smartt, S.J., Eldridge, J.J., Crockett, R.M and Maund, J.R., "The death of massive stars - I. Observational constraints on the progenitors of Type II-P supernovae", Mon. Not. R. Astron. Soc., 395, 1409-1437, (2008). [DOI], [arXiv:0809.0403]. (Cited on page 26.) 
[289] Smith, S.C., Houser, J.L. and Centrella, J.M., "Simulations of Nonaxisymmetric Instability in a Rotating Star: A Comparison Between Eulerian and Smooth Particle Hydrodynamics", Astrophys. J., 458, 236-256, (1996). [DOI], [ADS], [arXiv:gr-qc/9510014]. (Cited on pages 48 and 50.)

[290] Spruit, H.C., "Dynamo action by differential rotation in a stably stratified stellar interior", Astron. Astrophys., 381, 923-932, (2002). [DOI], [ADS]. (Cited on page 37.)

[291] Stark, R.F. and Piran, T., "Gravitational-Wave Emission from Rotating Gravitational Collapse", Phys. Rev. Lett., 55, 891-894, (1985). [DOI], [ADS]. (Cited on pages 32 and 63.)

[292] "Stellar Hydrodynamics", project homepage, Max Planck Institute for Astrophysics. URL (accessed 7 January 2009):

http://www.mpa-garching.mpg.de/hydro/index.shtml. (Cited on page 40.)

[293] Stergioulas, N., Apostolatos, T.A. and Font, J.A., "Non-linear pulsations in differentially rotating neutron stars: mass-shedding-induced damping and splitting of the fundamental mode", Mon. Not. R. Astron. Soc., 352, 1089-1101, (2004). [DOI], [ADS]. (Cited on page 40.)

[294] Stergioulas, N. and Font, J.A., "Nonlinear $r$-modes in rapidly rotating relativistic stars", Phys. Rev. Lett., 86, 1148-1151, (2001). [DOI], [ADS], [arXiv:gr-qc/0007086]. (Cited on page 57.)

[295] Sumiyoshi, K., Yamada, S. and Suzuki, H., "Dynamics and Neutrino Signal of Black Hole Formation in Nonrotating Failed Supernovae. II. Progenitor Dependence", Astrophys. J., 688, 1176-1185, (2008). [DOI], [ADS]. (Cited on page 27.)

[296] Sumiyoshi, K., Yamada, S., Suzuki, H. and Chiba, S., "Neutrino Signals from the Formation of a Black Hole: A Probe of the Equation of State of Dense Matter", Phys. Rev. Lett., 97, 091101, (2006). [DOI], [ADS]. (Cited on page 27.)

[297] Summerscales, T.Z., Burrows, A., Finn, L.S. and Ott, C.D., "Maximum Entropy for Gravitational Wave Data Analysis: Inferring the Physical Parameters of Core-Collapse Supernovae", Astrophys. J., 678, 1142-1157, (2008). [DOI], [ADS]. (Cited on page 67.)

[298] Suwa, Y., Takiwaki, T., Kotake, K. and Sato, Katsuhiko, "Gravitational Wave Background from Population III Stars", Astrophys. J., 665, 521078, L43-L46, (2007). [DOI], [ADS]. (Cited on page 67.)

[299] Symbalisty, E.M.D., "Magnetorotational Iron Core Collapse", Astrophys. J., 285, 729-746, (1984). [DOI], [ADS]. (Cited on pages 18 and 33.)

[300] Takiwaki, T. and Kotake, K., "Gravitational-Wave Signatures in Magnetically-Driven Supernova Explosions", Phys. Rev. D, submitted, (2010). [arXiv:1004.2896]. (Cited on page 36.)

[301] "TAMA: The 300m Laser Interferometer Gravitational Wave Antenna", project homepage, National Astronomical Observatory of Japan. URL (accessed 4 October 2002): http://tamago.mtk.nao.ac.jp/. (Cited on page 5.)

[302] Tassoul, J.-L., Theory of Rotating Stars, (Princeton University Press, Princeton, 1978). (Cited on pages 36 and 48.)

[303] Teukolsky, S.A., "Perturbations of a Rotating Black Hole. I. Fundamental Equations for Gravitational, Electromagnetic, and Neutrino-Field Perturbations", Astrophys. J., 185, 635647, (1973). [DOI], [ADS]. (Cited on page 7.) 
[304] Thompson, C. and Murray, N., "Transport of Magnetic Fields in Convective, Accreting Supernova Cores", Astrophys. J., 560, 339-357, (2001). [DOI], [ADS]. (Cited on page 18.)

[305] Thompson, T.A., Chang, P. and Quataert, E., "Magnetar Spin-Down, Hyperenergetic Supernovae, and Gamma-Ray Bursts", Astrophys. J., 611, 380-393, (2004). [DOI], [ADS]. (Cited on page 22.)

[306] Thorne, K.S., "Multipole expansions of gravitational radiation", Rev. Mod. Phys., 52, 299339, (1980). [DOI], [ADS]. (Cited on pages 7, 10, 33, and 43.)

[307] Thorne, K.S., "Gravitational radiation", in Hawking, S.W. and Israel, W., eds., Three Hundred Years of Gravitation, pp. 330-458, (Cambridge University Press, Cambridge; New York, 1987). [Google Books]. (Cited on page 63.)

[308] Thorne, K.S., "Gravitational radiation", in Böhringer, H., Morfill, G.E. and Trümper, J.E., eds., 17th Texas Symposium on Relativistic Astrophysics and Cosmology, Ann. N.Y. Acad. Sci., 759, pp. 127-152, (New York Academy of Sciences, New York, 1995). [DOI], [ADS]. (Cited on page 60.)

[309] Thorne, K.S., "Gravitational Waves from Compact Bodies", in van Paradijs, J., van den Heuvel, E.P.J. and Kuulkers, E., eds., Compact Stars in Binaries (IAU Colloquium 165), Proceedings of the 165th Symposium of the International Astronomical Union, held in The Hague, the Netherlands, August 15-19, 1994, pp. 153-183, (Kluwer Academic Publishers, Dordrecht; Boston, 1996). [ADS]. (Cited on page 5.)

[310] Thorne, K.S., Price, R.H. and MacDonald, D.A., eds., Black Holes: The Membrane Paradigm, (Yale University Press, New Haven, 1986). (Cited on page 7.)

[311] Thuan, T.X. and Ostriker, J.P., "Gravitational Radiation from Stellar Collapse", Astrophys. J. Lett., 191, L105-L107, (1974). [DOI], [ADS]. (Cited on pages 6 and 32.)

[312] Tohline, J.E., "The Collapse of Rotating Stellar Cores: Equilibria Between White Dwarf and Neutron Star Densities", Astrophys. J., 285, 721-728, (1984). [DOI], [ADS]. (Cited on page 33.)

[313] Tohline, J.E., Durisen, R.H. and McCollough, M., "The linear and nonlinear dynamic stability of rotating $n=3 / 2$ polytropes", Astrophys. J., 298, 220-234, (1985). [DOI], [ADS]. (Cited on page 48.)

[314] Tohline, J.E. and Hachisu, I., "The Breakup of Self-Gravitating Rings, Tori, and Thick Accretion Disks", Astrophys. J., 361, 394-407, (1990). [DOI], [ADS]. (Cited on pages 48 and 51.)

[315] Toman, J., Imamura, J.N., Pickett, B.K. and Durisen, R.H., "Nonaxisymmetric Dynamic Instabilities of Rotating Polytropes. I. The Kelvin Modes", Astrophys. J., 497, 370-387, (1998). [DOI], [ADS]. (Cited on page 48.)

[316] Toomre, A., "On the gravitational stability of a disk of stars", Astrophys. J., 139, 1217-1238, (1964). [DOI], [ADS]. (Cited on page 8.)

[317] Turner, M.S., "Gravitational radiation from supernova neutrino bursts", Astrophys. J., 274, 565-566, (1978). [DOI], [ADS]. (Cited on pages 11 and 54.) 
[318] Turner, M.S. and Wagoner, R.V., "Gravitational radiation from slowly-rotating 'supernovae': Preliminary results", in Smarr, L.L., ed., Sources of Gravitational Radiation, Proceedings of the Battelle Seattle Workshop, July 24 -August 4, 1978, pp. 383-407, (Cambridge University Press, Cambridge, 1979). [ADS], [Google Books]. (Cited on page 32.)

[319] van den Heuvel, E.P.J. and Yoon, S.-C., "Long gamma-ray burst progenitors: boundary conditions and binary models", Astrophys. Space Sci., 311, 177-183, (2007). [DOI], [ADS], [arXiv:0704.0659]. (Cited on pages 9 and 49.)

[320] van Putten, M.H.P.M., "Proposed Source of Gravitational Radiation from a Torus around a Black Hole", Phys. Rev. Lett., 87, 091101, (2001). [DOI], [ADS]. (Cited on page 8.)

[321] van Putten, M.H.P.M. and Levinson, A., "Theory and Astrophysical Consequences of a Magnetized Torus around a Rapidly Rotating Black Hole", Astrophys. J., 584, 937-953, (2003). [DOI], [ADS], [arXiv:astro-ph/0212297]. (Cited on page 58.)

[322] van Riper, K.A. and Arnett, W.D., "Stellar Collapse and Explosion: Hydrodynamics of the Core", Astrophys. J. Lett., 225, L129-L132, (1978). [DOI], [ADS]. (Cited on page 37.)

[323] Villain, L., Pons, J.A., Cerdá-Durán, P. and Gourgoulhon, E., "Evolutionary sequences of rotating protoneutron stars", Astron. Astrophys., 418, 283-294, (2004). [DOI], [ADS]. (Cited on page 50.)

[324] "Virgo", project homepage, INFN. URL (accessed 4 October 2002): http://www.virgo.infn.it. (Cited on page 5.)

[325] Walder, R., Burrows, A., Ott, C.D., Livne, E., Lichtenstadt, I. and Jarrah, M., "Evolutionary sequences of rotating protoneutron stars", Astrophys. J., 626, 317-332, (2005). [DOI], [ADS]. (Cited on pages 36 and 49.)

[326] Watts, A.L., Andersson, N. and Jones, D.I., "The Nature of Low $T /|W|$ Dynamical Instabilities in Differentially Rotating Stars", Astrophys. J., 618, L37-L40, (2005). [DOI], [ADS]. (Cited on pages 49 and 53.)

[327] Weinberg, N.A. and Quataert, E., "Non-linear saturation of g-modes in proto-neutron stars: quieting the acoustic engine", Mon. Not. R. Astron. Soc., 387, L64-L68, (2008). [DOI], [ADS], [arXiv:0802.1522]. (Cited on pages 22 and 45.)

[328] Wheeler, J.A., "Geometrodynamics and the Issue of Final State", in DeWitt, C.M. and DeWitt, B.S., eds., Relativity, Groups and Topology. Relativité, Groupes et Topologie, Lectures delivered at Les Houches during the 1963 session of the Summer School of Theoretical Physics, University of Grenoble, pp. 315-320, (Gordon and Breach, New York; London, 1964). (Cited on page 12.)

[329] Wheeler, J.C., Meier, D.L. and Wilson, J.R., "Asymmetric Supernovae from Magnetocentrifugal Jets", Astrophys. J., 568, 807-819, (2002). [DOI], [ADS]. (Cited on page 27.)

[330] Wickramasinghe, D.T. and Ferrario, L., "Magnetism in Isolated and Binary White Dwarfs", Publ. Astron. Soc. Pac., 112, 873-924, (2000). [DOI], [ADS]. (Cited on page 51.)

[331] Williams, H.A. and Tohline, J.E., "Linear and nonlinear dynamic instability of rotating polytropes", Astrophys. J., 315, 594-601, (1987). [DOI], [ADS]. (Cited on page 48.)

[332] Woodward, J.W., Tohline, J.E. and Hachisu, I., "The Stability of Thick, Self-gravitating Disks in Protostellar Systems", Astrophys. J., 420, 247-267, (1994). [DOI], [ADS]. (Cited on page 51.) 
[333] Woosley, S.E., "Gamma-ray bursts from stellar mass accretion disks around black holes", Astrophys. J., 405, 273-277, (1993). [DOI], [ADS]. (Cited on pages 5, 18, and 27.)

[334] Woosley, S.E. and Baron, E., "The collapse of white dwarfs to neutron stars", Astrophys. J., 391, 228-235, (1992). [DOI], [ADS]. (Cited on pages 25 and 26.)

[335] Woosley, S.E. and Bloom, J., "The Supernova-Gamma-Ray Burst Connection", Annu. Rev. Astron. Astrophys., 44, 507-556, (2006). [DOI], [ADS]. (Cited on page 18.)

[336] Yakunin, K.N. et al., "Gravitational waves from core collapse supernovae", Class. Quantum Grav., 27, 194005, (2002). [DOI], [ADS], [arXiv:1005.0779]. (Cited on page 18.)

[337] Yamada, S. and Sato, K., "Gravitational Radiation from Rotational Collapse of a Supernova Core", Astrophys. J., 450, 245-252, (1995). [DOI], [ADS]. (Cited on page 35.)

[338] Yoon, S.-C. and Langer, N., "Evolution of rapidly rotating metal-poor massive stars towards gamma-ray bursts", Astron. Astrophys., 443, 643-648, (2005). [DOI], [ADS]. (Cited on pages 9 and 49.)

[339] Yoon, S.-C. and Langer, N., "On the evolution of rapidly rotating massive white dwarfs towards supernovae or collapses", Astron. Astrophys., 435, 967-985, (2005). [DOI], [ADS]. (Cited on pages 9, 25, and 49.)

[340] Yoon, S.-C., Langer, N. and Norman, C., "On the evolution of rapidly rotating massive white dwarfs towards supernovae or collapses", Astron. Astrophys., 460, 199-208, (2006). [DOI], [ADS]. (Cited on pages 9 and 49.)

[341] Yoon, S.-C., Langer, N. and Scheithauer, S., "Effects of rotation on the helium burning shell source in accreting white dwarfs", Astron. Astrophys., 425, 217-228, (2004). [DOI], [ADS]. (Cited on pages 9, 25, and 49.)

[342] Yoon, S.-C., Podsiadlowski, P. and Rosswog, S., "Remnant evolution after a carbon-oxygen white dwarf merger", Mon. Not. R. Astron. Soc., 380, 933-948, (2007). [DOI], [ADS]. (Cited on pages 9,25 , and 49 .)

[343] Yoshida, S., Ohnishi, N. and Yamada, S., "Excitation of $g$-Modes in a Proto-Neutron Star by the Standing Accretion Shock Instability", Astrophys. J., 665, 1268-1276, (2007). [DOI], [ADS], [arXiv:astro-ph/0701643]. (Cited on pages 22 and 45.)

[344] Zanotti, O., Rezzolla, L. and Font, J.A., "Quasi-periodic accretion and gravitational waves from oscillating 'toroidal neutron stars' around a Schwarzschild black hole", Mon. Not. $R$. Astron. Soc., 341, 832-848, (2003). [DOI], [ADS]. (Cited on page 58.)

[345] Zel'dovich, Y.B. and Novikov, I.D., Relativistic Astrophysics, 1, (University of Chicago Press, Chicago, 1971). (Cited on page 29.)

[346] Zerilli, F.J., "Gravitational field of a particle falling in a Schwarzschild geometry analyzed in tensor harmonics", Phys. Rev. D, 2, 2141-2160, (1970). [DOI], [ADS]. (Cited on page 12.)

[347] Zhang, W., Woosley, S.E. and Heger, A, "Fallback and Black Hole Production in Massive Stars", Astrophys. J., 679, 639-654, (2008). [DOI], [ADS]. (Cited on page 27.)

[348] Zink, B., Stergioulas, N., Hawke, I., Ott, C.D., Schnetter, E. and Müller, E., "Formation of Supermassive Black Holes through Fragmentation of Torodial Supermassive Stars", Phys. Rev. Lett., 96, 161101, (2006). [DOI], [ADS]. (Cited on pages 8, 29, and 59.) 
[349] Zink, B., Stergioulas, N., Hawke, I., Ott, C.D., Schnetter, E. and Müller, E., "Nonaxisymmetric instability and fragmentation of general relativistic quasitoroidal stars", Phys. Rev. $D, \mathbf{7 6}, 024019$, (2007). [DOI], [ADS]. (Cited on page 59.)

[350] Zwerger, T. and Müller, E., "Dynamics and gravitational wave signature of axisymmetric rotational core collapse", Astron. Astrophys., 320, 209-227, (1997). [ADS]. (Cited on pages 6, $33,34,35,36,37,43,50,51$, and 52.) 